\title{
2 THEORETISCHER HINTERGRUND UND METHODEN
}

\subsection{Untersuchungsgebiet}

Der Biosphärenpark Wienerwald erstreckt sich auf einer Fläche von 105.645 ha in Form eines hügeligen Berglandes auf einer Seehöhe zwischen 200 und 890m. Das Gebiet lässt sich aus geologischer Sicht in zwei Bereiche teilen: den nördlichen Flysch-Wienerwald, auch Sandstein-Wienerwald genannt, und den südlichen Kalkstein-Wienerwald entlang der Thermenlinie (Auer, 1982). Im Gebiet des Biosphärenparks münden die nördlichen Ausläufer der südlich des Parks liegenden Alpen. Im Osten schließt das Wiener Becken an, im Norden das Weinviertel und im Westen das Alpenvorland.

Auf Basis der geologischen und klimatischen Gegebenheiten (subkontinental kalte Winter und trocken-heiße Sommer) und der damit verbundenen Bodenbonität ergibt sich ein von Buchen und Eichen-Hainbuchen dominiertes Waldbild mit insgesamt 20 Waldtypen. Im mitteleuropäischen Vergleich handelt es sich um eines der größten mehr oder weniger geschlossenen Laubwaldgebiete. Die Wälder befinden sich überwiegend in Großgrundbesitz; sie sind verglichen mit ähnlichen Lagen überdurchschnittlich alt und reich an Habitatelementen wie Totholz. Betreffend die Offenlandtypen sind 17 Wiesentypen erfasst; das Pflanzenarteninventar umfasst über 2000 Pflanzenarten. Der Lebensraum Wienerwald beherbergt rund 150 Brutvogelarten. Die ökologische und naturschutzfachliche Wertigkeit des Wienerwaldes wird durch die klimatische, geologische und biogeografische Grenzlage, durch gebietsweise häufig vorkommende Sonderstandorte und durch die enge Verzahnung zwischen Wald und wertvollen Offenlandbereichen zusätzlich gesteigert.

Die vergleichsweise hohe Biodiversität des Wienerwalds spiegelt sich auch in der Vielfalt jagdbarer (im Jagdrecht geregelter) und anderer Wildtierarten wider. Niederwildreiches Offenland wechselt mit großflächigen und weitgehend störungsarmen Waldbereichen, die insbesondere als Einstandsgebiete für wildlebende Huftierarten („Schalenwild“) von Bedeutung sind.

Überregional stellt er prinzipiell ein mögliches Trittsteinhabitat für den Alpen-Karpaten Korridor dar. Angesichts der Nähe zu Wien und zur Donau ist die Erhaltung der Durchlässigkeit ein wichtiges Anliegen.

Der Biosphärenpark umfasst 51 Gemeinden in Niederösterreich und 7 Wiener Gemeindebezirke. Auf der niederösterreichischen Seite haben drei Gemeinden mehr als 20.000 Einwohner. $60 \%$ haben dagegen weniger als 5.000 Einwohner. Der Biosphärenpark dient neben dem Schutz der Natur auch der wirtschaftlichen und sozialen Entwicklung der Region und der Erhaltung kultureller Werte. Er wird in Kernzonen, Pflegezonen und Entwicklungszonen gegliedert. Weiters umfasst der Biosphärenpark 15 Naturschutzgebiete und 4 Naturparke.

Die Entwicklungszone umfasst vorwiegend Siedlungs- und Wirtschaftsraum und dient neben wirtschaftlicher Entwicklung als Freizeit- und Erholungsraum für die Bevölkerung, für Besucher und Touristen. Für diese Zone gilt es, neue Wege zur nachhaltigen Bewirtschaftung der regionalen Ressourcen und eine Steigerung der Wertschöpfung zu entwickeln. Partnerschaften und Kooperationsformen zwischen Teilregionen, Gemeinden und Interessenbereichen sowie die Entwicklung einer regionalen Identität unter dem Prädikat „Biosphärenpark Wienerwald" sind das vorrangige Ziel.

Als Pflegezone werden insbesondere wertvolle Kulturlandschaften mit besonders hohem Wert für das Landschaftsbild und die Artenvielfalt zusammengefasst. Entstanden sind solche Gebiete durch rücksichtsvolle Bewirtschaftung und nachhaltige Nutzung. Ziel der Pflegezonen ist die Erhaltung bzw. Schaffung möglichst zusammenhängender Landschaftskomplexe aus Wiesen, Weiden, Äckern, Brachen, Wein- und Obstgärten, Gewässern, reichhaltigen 
Kleinstrukturen, etc. Die Wiederbelebung bzw. Etablierung nachhaltiger Nutzungsformen soll eine Korridorfunktion für Wildtiere übernehmen und Nutzungsdruck gegenüber den kleinflächigen Kernzonen abpuffern.

Der Naturschutz- und Erhaltungsfunktion gewidmet sind die 36 dezentral verteilten Kernzonen, die besonders wertvolle Waldbestände umfassen. Es handelt sich ausschließlich um bestockte Flächen, die in etwa $8 \%$ der gesamten Waldfläche entsprechen. Mehr als 20 verschiedene Waldgesellschaften wurden dabei berücksichtigt. Auf diesen Flächen erfolgt in Zukunft keine forstwirtschaftliche Nutzung - die Entwicklung von Naturwaldflächen mit möglichst geringer menschlicher Einflussnahme (Prozessschutz) ist hier das primäre Ziel.

Im Biosphärenpark Wienerwald entfallen rund $5 \%$ auf Kernzonen, etwa $19 \%$ sind als Pflegezone ausgewiesen. Die restlichen rund $76 \%$ der Fläche dienen als Entwicklungszone der nachhaltigen Entwicklung des Gebiets.

Die 1995 beschlossene "Sevilla-Strategie" stellt mit dem "Statutory Framework of the World Network of Biosphere Reserves" (UNESCO \& MAB-ICC, 1996) die weltweit verbindliche Grundlage für das Netzwerk der UNESCO-Biosphärenreservate dar. Der Biosphärenpark Wienerwald wurde 2005 durch die UNESCO anerkannt. Das gegenständliche Projekt IESP hat insbesondere starken Bezug zum zweiten und dritten Ziel der Sevilla-Strategie (UNESCO \& MAB-ICC, 1996):

- Entwicklung und Förderung von ökologisch, wirtschaftlich und sozio-kulturell nachhaltigen Formen der Landnutzung.

- Unterstützung von Forschung, Umweltbeobachtung und Bildungsaktivitäten für besseres Verstehen der Wechselwirkungen zwischen Mensch und Natur.

Eine weitergehende Charakterisierung des Untersuchungsgebietes im Hinblick auf die Ziele des gegenständlichen Projekts ist Reimoser et al. (2008) zu entnehmen.

Abbildung 1 zeigt die geographische Lage des Biosphärenparks in den beiden Bundesländern Niederösterreich und Wien. 


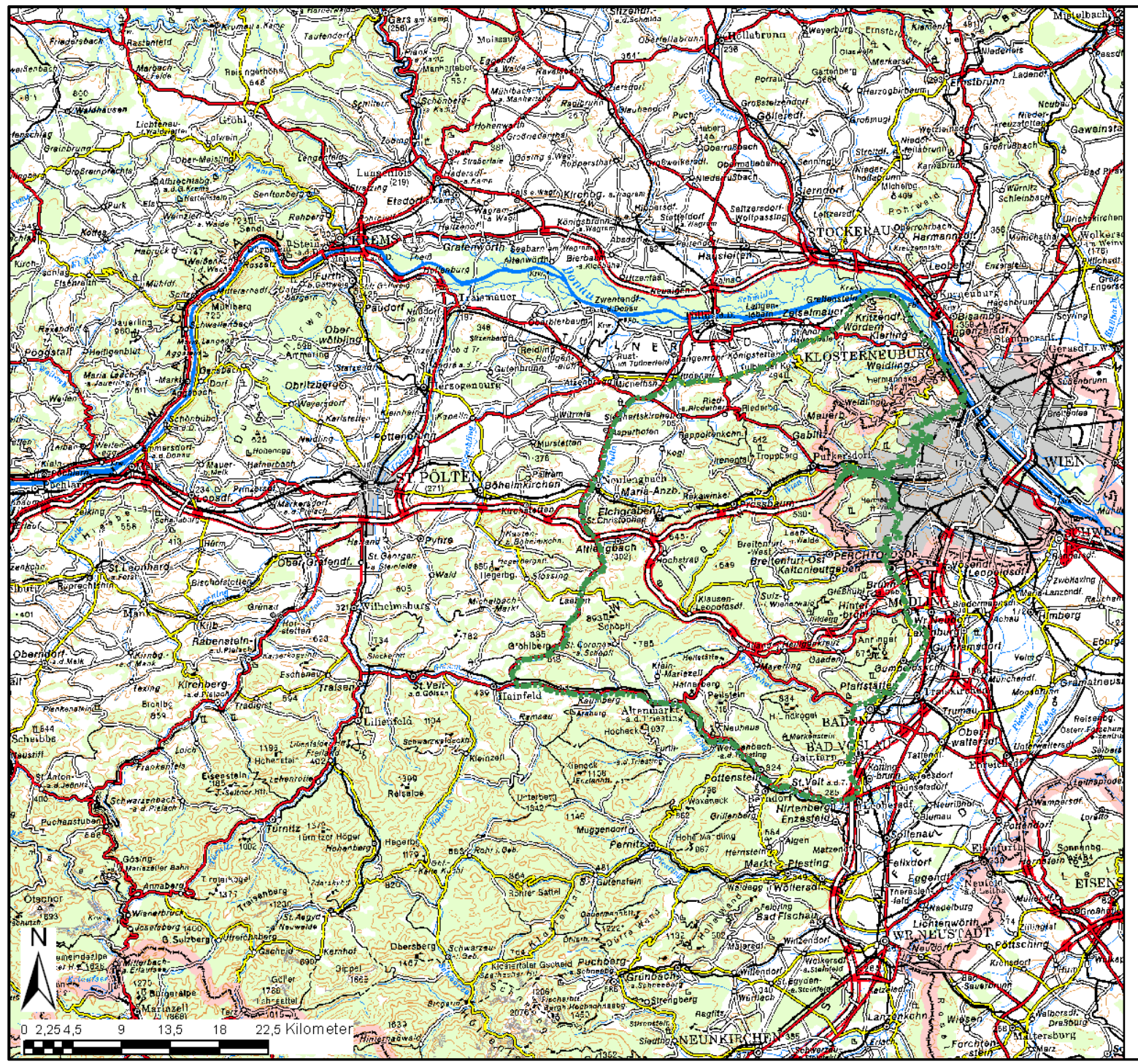

\section{Biosphärenpark Wienerwald}

Lage des Biosphärenparks in Niederösterreich und Wien

Grenze des BPWW

\begin{tabular}{|c|c|c|}
\hline & 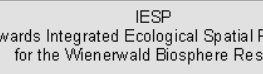 & \\
\hline 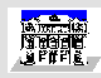 & 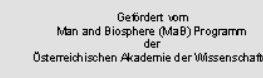 & Lensuserio \\
\hline 2 & umweltbundesamt $^{\circ}$ & \\
\hline \pm & & 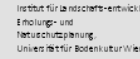 \\
\hline
\end{tabular}

Abbildung 1: Lage des Biosphärenparks in den Bundesländern Niederösterreich und Wien. 


\subsection{Theoretischer Hintergrund}

\subsubsection{Erholungseignung der Landschaft}

Die flächenbezogene Landschaftsbewertung hinsichtlich Erholungspotential und Erholungseignung erlebte von den 1970er bis zum Beginn der 1990er Jahre ihre Blütezeit. Die Zielsetzung der Bewertung bestand darin, die Erholungseignung von Landschaften in einem objektivierten, nachvollziehbaren Verfahren zu quantifizieren und damit Grundlagen für die Landschaftsplanung und Raumordnung zu legen (z.B. Ruppert, 1971). Entscheidende Impulse erhielt diese Forschungsrichtung durch die Dissertation von Kiemstedt mit dem Titel "Zur Bewertung der Landschaft für die Erholung“ (1967), in der Kiemstedt im Rahmen einer Nutzwertanalyse messbare Kennziffern wie Vielfältigkeitswert, Randeffekte und Reliefenergie, sowie quantitative Bewertungsverfahren zur objektivierten Beschreibung der Landschaftsstruktur erarbeitete. Auf die von Kiemstedt entwickelten Kriterien haben sich in der Folgezeit (und zum Teil bis heute) zahlreiche Autoren bezogen und diese immer wieder modifiziert und weiterentwickelt (z. B. Ruppert, 1971; Barsch \& Saupe et al. 1994; Greif, Pfusterschmied \& Wagner, 2002; Walz \& Berger, 2004). Bereits Ende der 1970er / Anfang der 1980er Jahre haben verschiedene Autoren vergleichende Analysen der zahlreichen Erholungsbewertungsmethoden und ihrer Bedeutung in der Landschafts-, Landes- und Regionalplanung durchgeführt (z.B. Pöttke, 1979; Harfst, 1980; Bonertz, 1981). Durch die Entwicklung geographischer Informationssysteme (GIS) in den 1960er Jahren wurde es zusätzlich möglich, eine flächendeckende Bewertung noch größerer Landschaftseinheiten mit einer weitaus höheren Vielfältigkeit an Auswertungsmöglichkeiten durchzuführen.

Jüngere Arbeiten zur Landschaftseignung sind daher am ehesten im Zusammenhang mit GIS-basierten, raumbezogenen Planungen von Tourismus und Erholungsnutzung zu finden. So etwa in Raumordnungsprogrammen und Regionalplänen, Landschaftsplänen oder Schutzgebietsplanungen (z.B. Engels, 200; Walz \& Berger, 2004; Zweckverband Großraum Braunschweig, 2008; Umweltatlas Dresden 2007).

Die Literaturrecherche zur Thematik der Erholungseignung hatte das Ziel, die Kriterien der unterschiedlichen in der Literatur genannten Bewertungsmehoden und -verfahren aufzuzeigen und so die Grundlage für die Bewertung des Wienerwaldes zu schaffen.

\subsubsection{Begriffsdefinition}

Landschaft wird auf diverse Arten genutzt. Eine Möglichkeit ist, sie zu Erholungszwecken aufzusuchen. Diese Form der Landschaftsnutzung erfreut sich immer größerer Beliebtheit, wodurch deren Bedeutung für die Raumplanung ebenfalls immer mehr zunimmt. Diese auf die Erholungsnutzung basierende Beziehung zwischen Mensch und Landschaft ist insofern etwas Besonderes, da sie kaum anfällig für streng ökonomische oder utilitaristische Erwägungen ist (Van der Zee, 1990). Zunächst bedarf es aber einer Definition der Begriffe „Landschaft“ und „Erholung“, um anschließend auf die Bedeutung der Erholungseignung der Landschaft eingehen zu können.

Der Begriff "Landschaft" wird gemäß der Europäischen Landschaftskonvention beispielsweise folgendermaßen definiert: "Landschaft umfasst entsprechend menschlicher Wahrnehmung ein Gebiet, dessen Beschaffenheit aus dem Handeln und Interagieren der natürlichen und/oder menschlichen Faktoren resultiert" (vgl. Council of Europe, 2000). In Österreich existieren 2.600 individuelle Landschaften, wie die Studie „Die Landschaften Österreichs und ihre Bedeutung für die biologische Vielfalt" von Wrbka et al. aus dem Jahr 2005 mittels der Auswertung von Satellitenbildkarten von Österreich zeigt. Dadurch, dass die verschiedenen 
Landschaften unterschiedliche Eigenschaften und Elemente aufweisen, können diese Landschaften auch ein unterschiedliches Maß an Erholungseignung und Attraktivität für Erholung besitzen. Bevor jedoch auf die Erholungseignung der Landschaft eingegangen werden kann, muss der Begriff der Landschaftseignung im Allgemeinen geklärt werden. Diesbezüglich wird auf die Definition der FAO (Food and Agriculture Organsiation) der Bewertung der Landschaftseignung aus dem Jahr 1985 zurückgegriffen: Die Bewertung der Landschaftseignung ist "the process of assessment of land performance when the land is used for specific purposes", also der Bewertungsprozess der Land(schafts)leistung, wenn das Land für einen bestimmten Zweck verwendet wird. Im Rahmen der Landschaftsbewertung ist das Landschaftsnutzungspotential sowohl von biophysikalischen und sozio-ökonomischen Bedingungen, sowie von Institutionen abhängig (Ekanayake \& Dayawansa, 2003).

Um auf die Landschaftseignung hinsichtlich der Erholungsnutzung eingehen zu können, muß nun noch der Begriff der Erholung erläutert werden. Der Deutsche Heilbäderverband definiert Erholung als „umgangssprachlichen Begriff für die spontane, primär nicht medizinisch gesteuerte Wiedererlangung (Rekompensation) körperlicher und seelischer Gleichgewichte, nach einseitiger Über- oder Unterforderung, in einer Entlastungssituation bei erhaltener Erholungsfähigkeit" (VHD, 2005). Van der Zee (1987) bezeichnet Erholung, basierend auf dem Konzept von Clawson \& Knetsch (1966), als "refreshment of body or mind by activities, or a planned inactivity, undertaken because one wants to do it, without any moral, economical, social or other pressure" (Van der Zee, 1987), also als Erfrischung von Körper oder Geist durch Aktivitäten oder durch geplante passive Regeneration, die nur aufgrund des eigenen Willens und ohne moralischen, ökonomischen, sozialen oder anderen Druck unternommen wird. Diese Definition des Begriffs Erholung umschließt eine Vielfalt von Aktivitäten, worunter aber auch Aktivitäten fallen, die im eigenen Heim durchgeführt werden können. Kurdoglu (2002) unterteilt den Begriff weiter in drei Bereiche: die Aktivitäten zu Hause, die IndoorAktivitäten an einem anderen Ort als in den eigenen vier Wänden und die OutdoorAktivitäten (Kurdoglu, 2002, zitiert in Gözde Kaya, 2006). Die Berg- und Naturwacht Österreich geht bei ihrer Definition nur auf den dritten von Kurdoglu (2002) genannten Erholungsbereich ein, also auf Aktivitäten in der Landschaft, weshalb sie die Erholung als die mit dem „Aufenthalt des Menschen in der Landschaft verbundene geistige und körperliche Regeneration" bezeichnet (www.bergundnaturwacht.at). Doch auch Van der Zee (1990) beschäftigt sich mit der Beziehung zwischen Erholung und Landschaft. Er nennt für Outdoor-Aktivitäten, also Erholungsaktivitäten die ausschließlich in der Natur unternommen werden, „Erholungsressourcen" als Schlüsselbegriff in der Erforschung der Beziehungen zwischen Erholung und Landschaft. So begeben sich Menschen zu Orten außerhalb ihres Heimes, da sie eine Form der Erholung suchen, die sie zu Hause nicht finden. Das Ziel der Reise wird durch Erholungsressourcen bestimmt: Manche benötigen einfach einen Ortswechsel, wodurch jeder Ort außerhalb der eigenen vier Wände angenommen wird. Andere aber suchen nach sonnigen Sandstränden, um sich zu sonnen, nach Bergen, um zu wandern, oder nach Seen, um zu segeln. Dies bedeutet allerdings nicht, dass jeder Sandstrand, Berg oder See eine Erholungsressource darstellt. Van der Zee (1990) definiert eine Landschaft vielmehr dann als Erholungsressource, wenn sie der Mensch als solche identifiziert und auch nutzt. Der Begriff der Erholungsressource taucht auch bei der Definition des Erholungswertes der Berg- und Naturwacht Österreich (www.bergundnaturwacht.at) auf: Erholungswert ist „die Bedeutung eines Gebietes für die Erholung des Menschen zum gegenwärtigen Zeitpunkt oder für die Zukunft (Erholungsressource)." Hier wird also nur die zukünftige Bedeutung der Landschaft für Erholungszwecke als Erholungsressource gesehen. Auf den Begriff „Erholungswert“ wird später noch genauer eingegangen.

Um Landschaften im Hinblick auf die touristische, Erholungs- und Freizeitnutzung zu bewerten, finden viele verschiedene Begriffe Anwendung. Drei von diesen oft synonym verwendeten Begriffen sind „Erholungseignung“, „Erholungswert“ und „Erholungspotential“, die jedoch häufig ohne eine genaue Begriffsklärung angewendet werden, oder lediglich über die ver-

IESP - Towards Integrated Ecological Spatial Planning for the Wienerwald Biosphere Reserve 
wendeten Kriterien definiert werden (z.B. Kiemstedt et al., 1975; Barsch \& Saupe et al., 1994; Grabaum et al., 2005, Mönnecke \& Wasem, 2005). Im Folgenden soll auf diese drei Begriffe im Detail eingegangen werden:

Das Erholungspotential gehört zu den Naturraumpotentialen Ertragspotential, Bebauungspotential, Entsorgungspotential) und beschreibt die Leistungsfähigkeit eines Naturraumes im Hinblick auf die Erholungsnutzung (vgl. Bruns, 2007). Diese dienen der Bewertung von naturräumlichen Eigenschaften, beziehungsweise von Landschaftseigenschaften und werden insbesondere in der Physiogeographie, Landschaftsökologie und Landschaftsplanung angewendet. Die Einbeziehung freizeitrelevanter Infrastruktur, wie sie in der folgenden Definition vorgeschlagen wird, stellt somit eine Erweiterung und Verfälschung des Potentialbegriffs dar: „Das Erholungspotential kennzeichnet das Vermögen des Naturraumes, der körperlichgeistigen Regenerierung und Entspannung und dem Genuss ästhetisch landschaftlicher Reize zu dienen. Neben landschaftsökologischen Faktoren und den Qualitäten des Landschaftsbildes wird in zunehmendem Maße die freizeitrelevante Infrastruktur in die Betrachtung des Erholungspotentials miteinbezogen." (Ökologische bzw. Ökologisch orientierte Planung - Lehrmaterial an der ETH Zürich, S. 7).

Der Begriff der Erholungseignung wird eher in der Planungspraxis verwendet. Sowohl in der Landschafts-, als auch in der Stadt- und Regionalplanung (Deutschland) stellt die Erholungseignung ein Instrument der Freizeit- und Erholungsplanung dar (z. B. Grundriss der Raumordnung 1982), das auf der Grundlage entsprechender Bewertungsverfahren die günstigen Eigenschaften einer Landschaft für die Erholungsnutzung darstellt. Eine Definition für Erholungseignung liefert der Grundriss der Raumordnung aber nicht. In den verschiedenen Plänen und Programmen werden zwar die Gebietsausweisungen, z.B. Vorranggebiete für Erholung und Tourismus, definiert, nicht aber die Erholungseignung oder das Erholungspotential, auf deren Basis die Bestimmung und Abgrenzung erfolgt (z. B. Zweckverband Großraum Braunschweig, 2008a). Im Regionalen Raumordnungsprogramm für den Großraum Braunschweig 2008 ist ein pragmatischer und eher normativer Umgang mit Erholungseignung erkennbar, da vor allem jene Gebiete als für die Erholung geeignet angesehen werden, die bereits Erholungsinfrastruktur bzw. Erholungsnutzung aufweisen. Diese Einschätzung lässt darauf schließen, dass der Begriff der Erholungseignung im regionalen Raumordnungsprogramm für den Großraum Braunschweig sehr stark mit einer vorrangigen Erholungsnutzung gegenüber anderen Nutzungen verknüpft ist und somit als die momentane Eignung der Landschaft hinsichtlich der Attraktivität für die Erholungsnutzungsausübung verstanden wird. Diese Vermutung wird von der Tatsache unterstützt, dass den bestehenden Naturparken ein besonderer Erholungswert beigemessen wird und dass neben dem landschaftlichen Erholungspotential der regionalen Freiräume und dem Städte-, Kultur- und Kongresstourismus die Lage zu Ober- und Mittelzentren und die Funktion für die unterschiedlichen Arten der Naherholung und des Ausflugsverkehrs berücksichtigt werden (vgl. Zweckverband Großraum Braunschweig, 2008a, 2008b).

Ein ähnlicher Zugang zur Thematik Erholungseignung wird auch im jüngsten Umweltatlas der Landeshauptstadt Dresden gewählt: „Die Wertvorstellungen hinsichtlich der Erholungseignung sind sehr vielschichtig. Einerseits entsteht aus der Charakteristik des Landschaftsraumes ein spezielles Potential, eine charakteristische Grundeignung und andererseits erfordern die vorhandenen Wohnstandorte bestimmte Eignungen und Funktionen. Neben den auf das jeweilige Wohnumfeld bezogenen Funktionen bestehen bei entsprechender Eignung auch übergreifende Erholungsfunktionen im Zuge der Naherholung. Aufgrund der Funktionsfülle müssen komplexe Indikatoren als Bewertungsmaßstab gewählt werden, die zum einen den speziellen landschaftsbezogenen Erholungs- und Erlebnispotentialen, und zum anderen den jeweiligen funktionalen Schwerpunkten gerecht werden." (Umweltatlas Dresden 5/2007 - Erläuterungen zur Karte 2.10 Bewertung der Erholungseignung, S. 1). Eine solche Auffassung von Eignung impliziert zugleich einen gewissen Vorrang der Erholungsnutzung bzw. - 
funktion gegenüber anderen Nutzungen. Nach Brandli \& Ulmer (2001) wird die Erholungseignung für einen Raum durch folgende fünf Kriterien bestimmt: Umwelteinflüsse (Naturgefahren, Emissionen), dem Landschaftspotential (Schönheit, Natürlichkeit, Diversität, Einzigartigkeit), Eignung für Aktivitäten (Typ und Intensität der Erholungsnutzung), räumliche Ausstattung mit Infrastruktur und Erreichbarkeit (Distanz oder Fahrzeit).

Im Raumordnungsbericht für Deutschland (2005) wird die Eignung eines ländlichen Raumes als Tourismusgebiet aus dem ursprünglichen und abgeleiteten Angebot, d.h. den landschaftlichen Voraussetzungen und der Fremdenverkehrsinfrastruktur begründet und die entscheidende Bedeutung der landschaftlichen Attraktivität für die Erholungs- und Tourismusfunktionen eines ländlichen Raumes hervorgehoben. Diese Eignung wird letztlich aber auf eine Verknüpfung von landschaftlicher Attraktivität und Nachfrage durch Mehrtagestourismus (Übernachtungen) zurückgeführt und es wird auf nationalstaatlicher Ebene der Index der touristischen und landschaftlichen Attraktivität ermittelt und dargestellt (Bundesamt für Bauwesen und Raumordnung 2005, S. 209). Engels (2008) ergänzt die Erläuterungen zu dieser Bewertung im Bundesraumordnungsbericht 2005 durch den Bezug zum Vielseitigkeitswert von Kiemstedt (1967) bei der Bewertung landschaftlicher Attraktivität und durch eine Reihe von Weiterentwicklungen wie die Einbeziehung von Störfaktoren und der Freizeitinfrastruktur (z.B. bei Ammer \& Pröbstl, 1991).

Im Lexikon Landschafts- und Stadtplanung wird Erholungseignung lediglich als „Benutzbarkeit der Landschaft nach qualitativen Kriterien, wie etwa hinsichtlich der leichten Erreichbarkeit für alle oder einzelne Bevölkerungsgruppen“ (Evert, 2001; S. 188) definiert, während Erholungspotential als „derzeitige und zukünftige Eignung von Landschaftsräumen oder Teilen davon für Freizeit und Erholung" aufgefasst wird (Evert, 2001; S. 190). Damit wird der Potentialbegriff stärker auf die zukünftige Entwicklung gelenkt. Der umfassendere Begriff ist hier der des Erholungswerts als „landschaftliche, ästhetische, informative und geistig belebende Gegebenheiten eines Erholungsraumes, abhängig von der natürlichen oder künstlichen Ausstattung, die das Erleben durch optische Reize oder Aktivitätsangebote steigert." (Evert, 2001; S. 191). Ein hoher Erholungswert kann zudem nur erreicht werden, wenn keine Störfaktoren wie Luft-, Lärm- und Geruchsimmissionen vorhanden sind (Evert, 2001; S. 191).

Erholungseignung und Erholungswert werden somit sehr ähnlich verwendet, wie auch folgende Definition zeigt: „Der Erholungswert eines Gebietes ist das Maß, in dem sich ein Gebiet zur Befriedigung des Erholungsbedürfnisses der Menschen eignet. Ein hoher Erholungswert ist gegeben, wenn verschiedene Landschaftselemente (Baumbestand, Wiesen, Felder, Gewässer und topographische Gliederung) geringe Immissionen (Lärm, Staub, Abgase), eine leichte Zugänglichkeit (Erreichbarkeit) und ein ausgewogenes Maß an Erschließung (Wanderwege und sonstige Einrichtungen) vorhanden sind." (Forum Umweltbildung: LandschaftsBildungsLandschaft - Glossar). Beide Begriffe werden von einigen Autoren auf bestimmte Ansprüche, Erholungsformen oder Freizeitaktivitäten bezogen. Walz \& Berger (Projekt Langzeitmonitoring von Flächennutzungsänderungen des Leibniz-Instituts für ökologische Raumentwicklung Dresden) setzten beispielsweise den Erholungswert in Bezug zu den unterschiedlichen Ansprüchen der verschiedenen Erholungsformen bzw. Freizeitaktivitäten (vgl. Walz \& Berger, 2004; keine Seitenangabe). In gleicher Weise wird auch in Kiemstedt et al. (1975) die Erholungseignung der Landschaft für eine Reihe ausgewählter Erholungsaktivitäten untersucht und jeweils das Mindestanspruchsniveau einer Erholungsaktivität zur Bewertung herangezogen.

\section{Fazit: Begriffe Erholungspotential, Erholungseignung, Erholungswert}

Abbildung 2 gibt einen Überblick über die Begriffe Erholungspotential, Erholungseignung und Erholungswert. Sowohl der Begriff des Erholungspotentials, als auch der der Erholungseig- 
nung werden in der Literatur je nach Verwendung und Kriterien unterschiedlich verwendet, während der Begriff des Erholungswertes relativ allgemein eingesetzt wird.

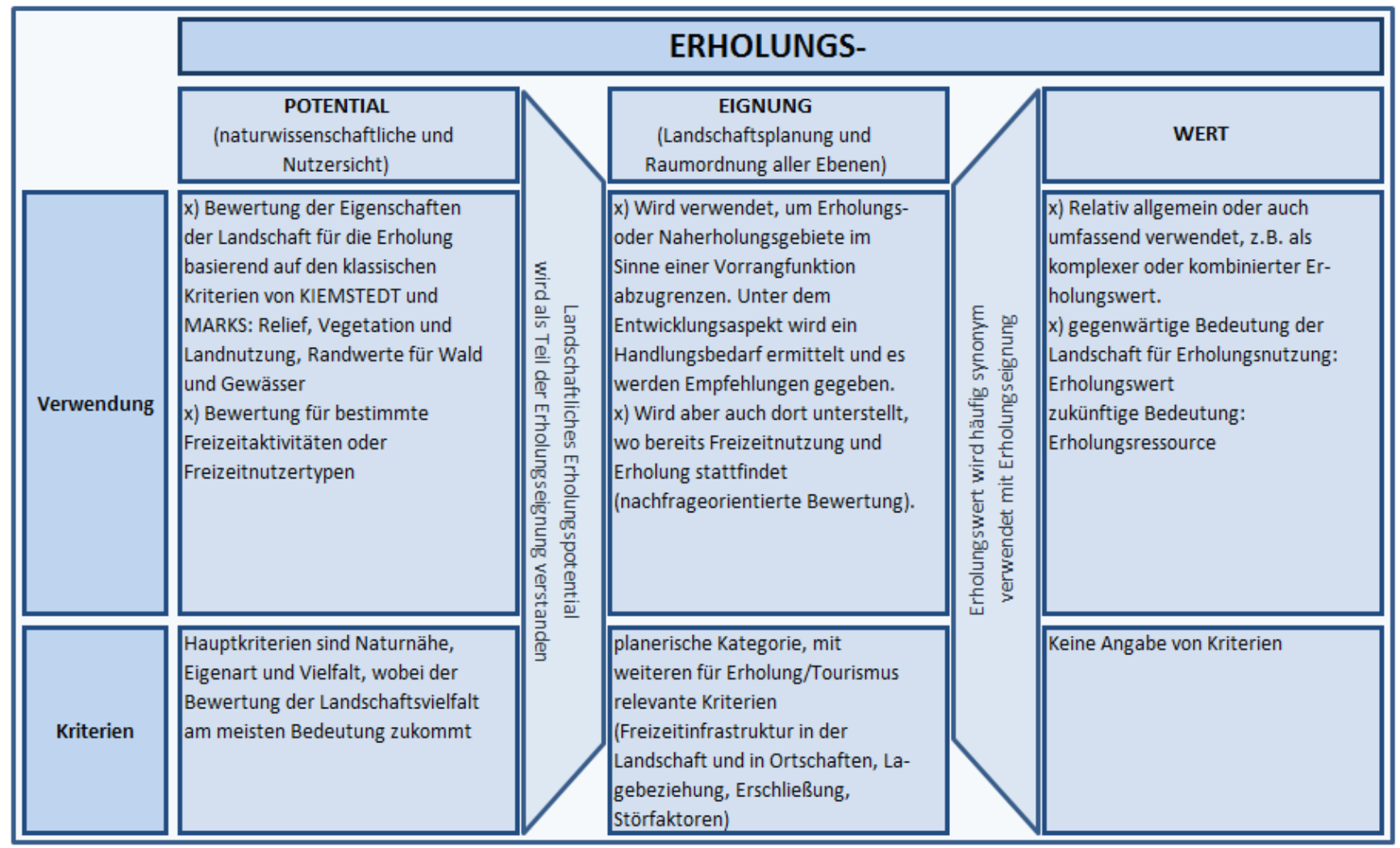

Abbildung 2: Überblick über die in der Literatur verwendeten Definitionen der Begriffe Erholungspotential, Erholungseignung und Erholungswert( eigene Darstellung).

Die Begriffsbestimmungen sind allerdings insgesamt sehr unscharf gehalten und werden häufig verwendet, ohne auf ihre genaue Bedeutung einzugehen, oder werden nur über die verwendeten Kriterien erklärt. Teilweise werden bereits in das landschaftliche Erholungspotential bzw. die landschaftliche Erholungseignung Kriterien der infrastrukturellen Ausstattung, der Erreichbarkeit u. ä. einbezogen. Ein Grund hierfür ist vermutlich die sehr komplexe Beziehung zwischen Landschaft und Erholung. Bedingt durch diese Komplexität, wurden auch verschiedene, miteinander verbundene Bewertungsansätze entwickelt. Diese unterschiedlichen Bewertungsverfahren werden im folgenden Kapitel vorgestellt.

\subsubsection{Bewertungsverfahren zur Erholungseignung}

Bereits Harfst (1980) untersuchte die große Fülle von Landschaftsbewertungsverfahren zur Erholungs- und Freizeitfunktion in der Landschaftsplanung (ca. 65 Verfahren). Als Klassifizierungsmöglichkeiten unterscheidet er insbesondere Nutzerbewertungsmethoden (ausgehend von der Nachfrage direkter und indirekter Nutzen der Landschaft) und Eignungsbewertungsmethoden (ausgehend vom räumlichen Angebot Eignung für Freizeitfunktionen), nutzerunabhängige und nutzerabhängige Eignungsbewertungen (Art der Erfassung), Partial- und Quasi-Total-Methoden (Komplexitätsgrad) sowie eine allgemeine und differenzierte Erholungsnutzung. 
So dominieren etwa bei den Zielsetzungen der nutzerunabhängigen Eignungsbewertungen ästhetisch-psychologische Zielsetzungen zur Beurteilung der Qualität des Landschaftsbildes und aktivitätsbezogene Zielsetzungen, bei denen Möglichkeiten der Freizeitausübung berücksichtigt werden. Häufig wurden Bewertungsverfahren nicht für eine flächendeckende Eignungsbewertung der gesamten Landschaft, sondern für bestimmte Landschaftselemente oder Landschaftsräume (z.B. Seengebiete, städtische Gebiete) entwickelt. Erholungseignungsbewertungen wurden auf allen Planungsebenen durchgeführt (vom Teilbereich einer Gemeinde bis zur nationalen Ebene). Die Erhebungs- und Bewertungsverfahren unterscheiden sich dementsprechend. Kritisiert werden von Harfst (1980) vor allem der im Vergleich zum Nutzung vielfach sehr hohe Aufwand, insbesondere wenn das empirische Datenmaterial komplizierte Erhebungs- und Bewertungsverfahren nicht rechtfertigt, und die Annahme einer generellen Übertragbarkeit. Bei der Frage der Vollständigkeit und Treffsicherheit konstatiert Harfst eine Fokussierung auf natürliche Gegebenheiten und infrastrukturelle Ausstattung, während die Bedarfsseite und aufgrund der mathematischen Ausrichtung auch die emotionalen Bindungen des Nutzers zu wenig berücksichtigt werden. Zudem vermisst Harfst die Einbeziehung der Nutzerbedürfnisse, so dass Weiterentwicklungen vor allem in der Modifizierung der Kriterienlisten bestehen, vor allem durch sozialstrukturelle und infrastrukturelle sowie in geringem Umfang auch nachfragbezogene Daten. Nutzerabhängige Verfahren zur Landschaftsbewertung zielen auf direkte Erfassung der aktuellen Nachfrage und der Verhaltensweisen der Erholungsuchenden und Freizeitnutzer in der Landschaft (Zählungen und Beobachtungen) oder eine indirekte Erhebung von Einstellungen oder Reaktionen anhand von Simulationsmaterialverfahren (Befragungen, psycho-physikalische Messungen) und leisten damit nur indirekt einen Beitrag zur Bewertung der Erholungseignung einer Landschaft (Harfst, 1980; S. 16-99).

\subsection{Bewertungsverfahren zur landschaftlichen Erholungseignung bzw. natur- räumlichen Erholungspotenzialen}

Reduziert man die Erholungseignung zunächst auf eine Bewertung naturräumlicher bzw. landschaftlicher Potenziale, so stößt man in den meisten Bewertungsverfahren auf die klassischen Kriterien von Kiemstedt oder Marks (z.B. Barsch \& Saupe et al., 1994; Grabaum et al., 2005; Walz \& Berger, 2004). Dabei werden in der Regel verschiedene Kriterien wie Naturnähe, Landschafts- bzw. Nutzungsvielfalt, Vorhandensein von Gewässern einbezogen und mehrere Indikatoren bzw. Parameter verwandt. Mit dem Einsatz computergestützter Verfahren wurde die Zahl der verwendeten Parameter zumeist noch erhöht. In Tabelle 1 sind die Hauptkriterien der landschaftlichen Erholungseignung sowie zugehörige Kriterien und Indikatoren zusammen-gestellt, die in Bewertungsverfahren Anwendung gefunden haben.

Tabelle 1: Landschaftliche Erholungseignung (abhängig von Maßstabsebene).

Hauptkriterien

landschaftliche Vielfalt

\section{Kriterien / Indikatoren}

\author{
Relief-Vielfalt: \\ Hangneigung, Reliefenergie, Gestaltreichtum, \\ Reliefklassen aus Hangneigung, Exposition und Wölbung, \\ Flächenneigung (20\%), enge Tallagen), Wiesentäler (km), \\ Bergkuppen (Anzahl), Reliefenergie (m), \\ landschaftsbedingten Reliefvielfalt \\ Landnutzung / Flächennutzung: \\ Klassifizierung der Landnutzungsarten, Vielfalt und/oder Ge- \\ wässernähe der Pflanzendecke,
}




\begin{tabular}{|c|c|}
\hline Hauptkriterien & Kriterien / Indikatoren \\
\hline & $\begin{array}{l}\text { Vielfalt der Flächen, } \\
\text { Kleinteiligkeit, mittelgroße Felder, Wechsel in den Land- } \\
\text { schaftselementen und Räumen, } \\
\text { Lichtungen im Wald (ha), Gewässerrandvegetation, } \\
\text { Diversität der Nutzungsformen }\end{array}$ \\
\hline & $\begin{array}{l}\text { Flächenform: } \\
\text { Landschaftsstrukturmaß für die Komplexität der Flächen und } \\
\text { den unregelmäßigen Verlauf der Ränder }\end{array}$ \\
\hline & $\begin{array}{l}\text { lineare Strukturelemente: } \\
\text { Wald- und Gewässerränder, Baumreihe, Hecken, Bachver- } \\
\text { lauf, } \\
\text { Gewässer- und Vegetationsränder, } \\
\text { Grenzverlauf (Länge je Flächeneinheit) } \\
\text { Randeffekte (Grad der Verzahnung verschiedener Kulturar- } \\
\text { ten), } \\
\text { Eigenschaften von Gewässerrändern, } \\
\text { Waldrandläge (km) }\end{array}$ \\
\hline & $\begin{array}{l}\text { punktuelle Strukturelemente: } \\
\text { Gliederung der Flächen, } \\
\text { Ausstattung mit Landschaftselementen, } \\
\text { Einzelbäume oder Baumgruppen auf Acker-, Wiesen-, Heide- } \\
\text { flächen, } \\
\text { viele Biotopelemente (Kleingewässer, Feldgehölze, Hecken), } \\
\text { Anzahl der punktförmigen Landschaftselemente } \\
\text { Acker mit Einzelbäumen, Acker mit Baumgruppen }\end{array}$ \\
\hline \multirow[t]{2}{*}{ Naturnähe / Natürlichkeit } & $\begin{array}{l}\text { Landnutzung / Bodenbedeckung: } \\
\text { Anteil unbebauter Flächen, } \\
\text { Hemerobiestufen, } \\
\text { Gewässer- und Vegetationsränder, } \\
\text { Flächenform (Unregelmäßigkeit = Natürlichkeit), } \\
\text { Stärke der anthropogenen Umgestaltung }\end{array}$ \\
\hline & $\begin{array}{l}\text { Zerschneidungsgrad als Ausdruck der Natürlichkeit / Nicht- } \\
\text { Natürlickeit, }\end{array}$ \\
\hline Wald & $\begin{array}{l}\text { Bewaldungsgrad, } \\
\text { hoher Waldanteil, } \\
\text { Anteil von Laub-, Nadel- und Mischwald (Anteil an Fläche) }\end{array}$ \\
\hline Gewässer & $\begin{array}{l}\text { Vorhandensein bzw. Fehlen von Gewässern, } \\
\text { See / Stausee (Anteil an Fläche), Fluss / Kanal, Bach / Graben } \\
\text { (Anzahl), Gewässergütestufen, Tiefenstufen und Exposition } \\
\text { von Gewässern, Eigenschaften von Gewässerrändern, }\end{array}$ \\
\hline Panoramawert & $\begin{array}{l}\text { Aussichten / Blickbeziehungen, } \\
\text { Sichtbarkeitsanalyse, } \\
\text { für Erholungsuchende besonders geeignete Aufenthaltsorte } \\
\text { bieten Sichtschutz und Aussicht zugleich, } \\
\text { Fernsicht }\end{array}$ \\
\hline Klima & Klimatische Höhenlage des Ortes (Stufen), \\
\hline
\end{tabular}




\begin{tabular}{ll} 
Hauptkriterien & Kriterien I Indikatoren \\
\hline & Jahresmitteltemperatur und mittlere jährliche Niederschläge, \\
& Bioklima \\
\hline Landschaftsbild & Qualität des Landschaftsbildes, \\
(visuell) & Ausstattung mit erholungswirksamen Elementen, \\
& Empfangbarkeit der jeweils landschaftsspezifischen Erlebnis- \\
& und Erholungswerte \\
starker Eindruck, in sehr großem Maße vorhanden, sehr auffäl- & lig, sehr wichtige Landschaftskomponente / schwach vorhan- \\
den, visuell oder akustisch wenig wirksam, wenig auffällige & Landschaftskomponente, wenig typisch
\end{tabular}

Quelle: Eigene Zusammenstellung nach Grabaum et al. (2005), Walz \& Berger (2004), Kiemstedt et al. (1975), Barsch \& Saupe et al. (1994), Raumordnungsbericht Deutschland (2005), Greif, Pfusterschmid \& Wagner (2002), Mönecke \& Wasem (2005), Umweltatlas Dresden (2007).

Im Folgenden werden einige Bewertungsverfahren vorgestellt, die sich auf die Bestimmung naturräumlicher und landschaftlicher Indikatoren für die Erholungseignung konzentrieren bzw. beschränken.

Der Ansatz von Kiemstedt (1967) wird bereits von Harfst (1980) als der einzige Bewertungsansatz hervorgehoben, der eine breitere Anwendung gefunden hat und immer wieder modifiziert worden ist. Bis heute beziehen sich zahlreiche Erholungseignungsverfahren insbesondere auf den Vielfältigkeitswert von Kiemstedt (1967). Dieser setzt sich aus dem Anteil von Wald- und Gewässerrändern, der Reliefenergie und dem Erholungswert der einzelnen Nutzungsarten zusammen. Dabei sind die Erholungsmöglichkeiten umso größer, desto extensiver die Flächen genutzt werden. Beim Klima werden aus der mittleren Jahrestemperatur und der mittleren jährlichen Niederschlagsmenge für die verschiedenen Höhenstufen Klimafaktoren berechnet. Die Berechnung erfolgt mittels der Division der Summe der Randzahlen, der Reliefenergie und der Nutzungszahl durch 1000 und der Multiplikation mit dem Klimafaktor (dargestellt in Pötke, 1979; S. 12-16).

Das Verfahren zur Bewertung der natürlichen Erholungseignung von Marks et al. (1992) wird in Abbildung 3 dargestellt. Auch hier fließen die Hauptkriterien der Vegetations- und Gewässerrandeffekte ein, das Relief, sowie die Flächennutzung. 


\section{Übersicht zum Verfahren von Marks (1992)}

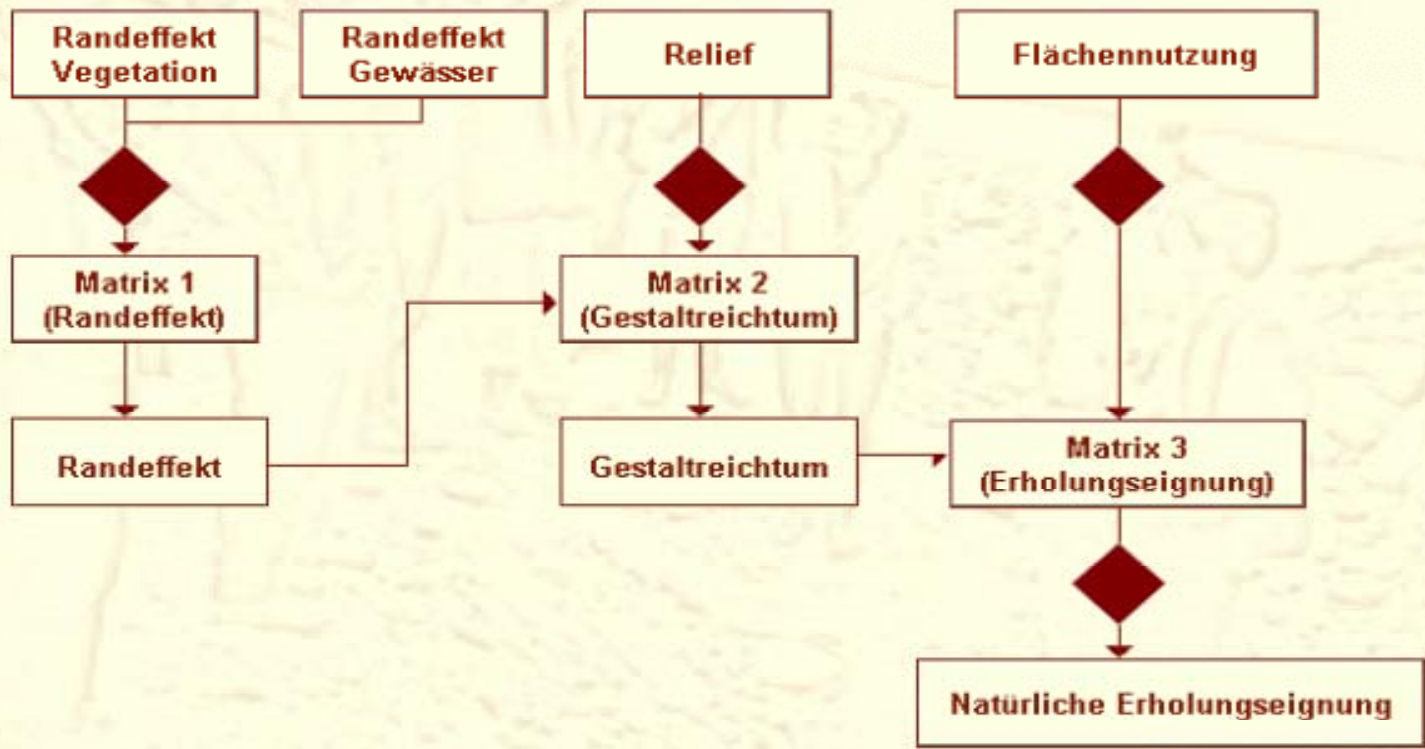

Abbildung 3: Bewertung der natürlichen Erholungseignung von Marks (1992).

Quelle: Grabaum et al. (2005), S. 2, unter Bezug auf Marks, R., M.J. Müller, H. Leser \& H.-J. Klink (Hrsg.) (1989).

Eine weitere Methode zur Bestimmung landschaftlicher Erholungseignung bezüglich des Kriteriums der Naturnähe wird in dem „Interaktiven Bewertungshandbuch- Sozioökonomische Bewertungsverfahren" das Verfahren von Bastian (1993, dargestellt von Weber \& Wardenga, 1999; in Bastian \& Schreiber), vorgestellt. Dabei werden fünf unterschiedliche Landschaftstypen (abgegrenzt nach physiognomischen Merkmalen) hinsichtlich verschiedener Kriterien untersucht:

- Technogene/urbane Gebiete,

- Agrargebiete,

- Wald-Agrar-Mischgebiete mit Waldanteil von bis zu 50 Prozent,

- waldbestimmte Gebiete, sowie

- stillgewässerbestimmte Gebiete.

Die Bewertungsmethode ist auf die Maßstabsebene 1:50000 ausgerichtet und lässt sich nur bedingt für eine großmaßstäbige Bewertung nutzen. Als Bewertungskriterien wurden der Natürlichkeitsgrad, die Stärke der anthropogenen Umgestaltung, das Vorhandensein bzw. Fehlen von Gewässern, die Diversität der Nutzungsformen, sowie die landschaftsbedingten Reliefvielfalt herangezogen. Das Bewertungsverfahren der Landschaftseinheiten erfolgte an- 
schließend mittels Delphi-Methode im Hinblick auf die Erholungsarten Wandern, Spazierengehen, Radfahren, Landschaftserleben, wasserbezogene Erholungsarten, Beobachtung der Pflanzen- und Tierwelt (Grabaum et al., 2005; S. 11).

Im Projekt „Langzeitmonitoring von Flächennutzungsänderungen: Auswirkungen des Landschaftswandels auf die Erholungseignung" des Leibniz-Instituts für ökologische Raumentwicklung Dresden (Projekthomepage Langzeitmonitoring, Walz \& Berger, 2004) wird ebenfalls auf landschaftliche Faktoren der Erholungseignung bzw. des Erholungspotentials fokussiert. Da das Untersuchungsgebiet Sächsische Schweiz eine Nationalparkregion ist, wird das Landschaftspotential für eine naturbezogene Erholung, das heißt die Eignung für die Erholungsaktivitäten wie Spazierengehen oder Wandern bewertet (Bezug auf Marks et al., 1992). Neben den genannten Erholungsnutzungen fließen weiters alle Zeitschnitte und auch alle Dörfer und Städte in die Bewertung ein, wobei durch die Verwendung von wenigen aussagefähigen Parametern die Gültigkeit des Bewertungsverfahrens erhöht und die Transparenz und Nachvollziehbarkeit gewährleistet werden soll.

Als Datengrundlage fungiert ausschließlich ein Geoinformationssystem, in dem das gesamte Bewertungsverfahren rasterbasiert (Rasterweite von $250 \mathrm{~m}$ ) durchgeführt wird. Für die Bewertung werden die beiden Parameter Naturnähe $(N)$ und Abwechslungsreichtum $(A)$ als zentrale Faktoren für naturbezogene Erholung angesehen (Vergleich verschiedener Bewertungsverfahren und Bezug auf (Kiemstedt 1967, Harfst 1980, Hoisl et al. 2000).

Daraus wurden folgende Parameter abgeleitet:

- Natürlichkeitsgrad der Flächennutzungen bzw. Bodenbedeckungen ( $\mathrm{N}$ - Hemerobiestufen als Grad des menschlichen Einflusses),

- Freiraumanteil (N - Anteil unbebauter Flächen),

- Randeffekt (N / A - Gewässer- und Vegetationsränder),

- Flächenform (N / A - Landschaftsstrukturmaß Shape Index als Maß für die Komplexität der Flächen bzw. den unregelmäßigen Verlauf der Ränder)

- Reliefdiversität (A (Landschaftsvielfalt) - Reliefklassen aus Hangneigung, Exposition und Wölbung),

- Überblick - Aussichten, Blickbeziehungen, die zum Erkunden und Interpretieren der Landschaft anregen, bei der Orientierung helfen, Gefühl der Sicherheit vermitteln (Relief und Flächennutzung - Sichtbarkeitsanalyse).

- Die Aggregation der Parameter erfolgte anschließend durch die einfache Addition der normierten Parameterwerte (0 bis 1 ) pro Bezugseinheit.

Ein Bewertungsverfahren das sich im Wesentlichen auf ein Landschaftskriterium beschränkt, ist das Verfahren von Grosjean (1986) zur ästhetischen Bewertung ländlicher Räume. Hierbei wird das Landschaftsbild nicht auf der Grundlage von Messwerten, sondern aus Sicht unterschiedlicher Typen von Erholungsuchenden beurteilt. Ein Set von Landschaftsmerkmalen (Oberfläche/Relief, Gletscher/Gewässer, Vegetation -Wald, offene Naturvegetation, Kulturvegetation-, Tierwelt, gebaute Umwelt -historischer Baubestand, neuerer angepasster Baubestand, neuerer kontrastierender Baubestand, Mischbebauungen, Industrie, Kulturtechnik, Verkehr- sowie der psychologischer Erlebniswerterfolgte) wird zunächst für vier Nutzertypen (Gewichtungsprofile) - Naturtyp, Traditionstyp, Aktivitätstyp, Promeniertyp durchgeführt. Jedes Landschaftsmerkmal wird auf einer Skala, die von $4=$ starker Eindruck, in sehr großem Maße vorhanden, sehr auffällig, sehr wichtige Landschaftskomponente bis zu 1 = schwach vorhanden, visuell oder akustisch wenig wirksam, wenig auffällige Landschaftskomponente, wenig typisch reicht beurteilt und nach den Gewichtungsprofilen gewich- 
tet. Anschließend erfolgte die Addition der gewichteten Werte zu einer Gesamtpunktzahl für jede Landschaftseinheit und die Zuordnung zu einer Klasse. Die Methode ist für großmaßstäbige Bewertungen (1:25000) geeignet, erfordert aber einen sehr hohen Aufwand. (Grabaum et al., 2005; S. 10).

\subsection{Bewertungsverfahren zur Erholungseignung von Waldgebieten}

Wald gilt als eines der Hauptkriterien für die Erholungseignung einer Landschaft. Ein hoher Waldanteil und insbesondere Laub- oder Mischwald tragen in Mitteleuropa wesentlich zur Attraktivität einer Landschaft bei. Entscheidend ist aber auch hierbei wiederum die Abwechslung und Landschaftsvielfalt, die sich beispielsweise in unterschiedlichen Altersklassen, dem Wechsel von Laub- und Nadelwald sowie von Wald und Wiese zeigt. Bewertungsverfahren, die die Erholungseignung in Waldgebieten bestimmen und differenzieren haben für das vorliegende Projekt zum Biosphärenpark Wienerwald somit eine besondere Bedeutung.

Eine sehr umfassende und komplexe Bewertung des Erholungswertes eines Waldgebietes hat Ruppert (1971) durchgeführt. Dabei wurde die Erholungsfunktion des Waldes in Naherholungsgebieten beurteilt. Die Grundlage bilden daher nicht nur naturräumliche Eigenschaften, sondern auch Lagebeziehungen und Störfaktoren. Der Erholungswert des Waldgebietes setzt sich aus den Gebietseigenschaften des Waldareals (Qualität des Angebots) und dem Nachbarschaftsverhältnis zu Siedlungen und anderen Waldflächen (Quantität von Nachfrage und Angebot) zusammen, wobei die Waldflächen durch Verkehrslinien begrenzt sind und eine Mindestgröße von 50 ha (ab 500 ha erfolgt eine Teilung nach Abteilungen) haben. Die Gebietseigenschaften bestehen wiederum aus erholungswirksamen Merkmalen der Naturausstattung und erholungsmindernden Merkmalen. Betrachtet wird bei den erholungswirksamen Merkmalen die Intensität der Einwirkung auf den Besucher als Träger sinnlicher Eindrücke, durch tonische Einflüsse und durch die Benutzbarkeit (stark, mittel, gering).

Zu den Gebietseigenschaften als erholungswirksame Merkmale (Naturraumausstattung) gehören:

- Waldrand:

Randlänge (m) im Verhältnis zur Fläche des Waldes (ha), angrenzende Nutzungsart (Multiplikationsfaktor), Aufbau des Waldrandes bzw. Störungen des Aufbaus,

- Baumartenzusammensetzung:

Baumartenzusammensetzung in den Einheiten der Zustandserfassung (Mischungsverhältnis), Wechsel der Baumarten in verschiedenen Einheiten der Zustandserfassung,

- Waldaufbau:

Anziehungskraft der Waldaufbauformen in den Einheiten der Zustandserfassung, Wechsel der Waldaufbauformen in den verschiedenen Einheiten der Zustandserfassung,

- Oberflächengestalt:

relative Höhenunterschiede, tonische (einflussmäßige) Wirkungen der Oberflächengestalt (Bioklima).

Als erholungsmindernde Merkmale werden akustische Störungen (Messstellen) und optische Störungen (Grundfläche flächenhafter und bandförmiger Störquellen) einbezogen. Schließlich werden die Nachbarschaft zu Siedlungen (Erreichbarkeit von den Quellgebieten) und die Nachbarschaft zu Waldflächen (,konkurrierende“ Erholungsflächen) berücksichtigt. Datengrundlagen für die Bewertungen sind die Forstgrundkarte 1:5000, die Schwarzgrundkarte 
1:10000, topographische Karten 1:25000 (Messtischblatt) sowie Erhebungen und Lautstärkenmessungen vor Ort.

Ein aktuelleres Projekt zur Erholungseignung in Waldarealen wurde von Kliskey (2000) durchgeführt. Kliskey entwickelte unter dem Titel "Recreation terrain suitability mapping: a spatially explicit methodology for determining recreation potential" eine räumlich explizite Methode, die eine Bestimmung des Erholungsgpotentials ermöglicht. Basierend auf einem geographischen Informationssystem (GIS), entstand so durch einen Eignungsindex für die Abgrenzung der räumlichen Ausdehnung eines möglichen Erholungsgeländes in der Landschaft (RTSI) eine Kartierungsmöglichkeit für die Erholungseignung. Das Verfahren basiert auf digitalen topographischen Karten, sowie auf digitalen Waldbestandskarten unter Berücksichtigung von Wildtierhabitaten und bewegt sich auf der Maßstabsebene von 1:20 000.

Die Methode umfasst vier Stufen: Zunächst wurden die Variablen (Offenheit des Waldes, Abgelegenheit, Hanglage, Erreichbarkeit, Topographie, Schneelage) auf einer Erholungseinstellungsskala für die Erholungsaktivität mittels Hauptkomponentenanalyse (PCA) identifiziert. Als nächster Schritt wurde ein Erholungseignungsmodell mit Hilfe der Raumkriterien der einzelnen Eignungsvariablen entwickelt (Tabelle 2).

\begin{tabular}{|c|c|c|c|c|c|}
\hline \multirow[t]{2}{*}{ Variable } & & \multicolumn{4}{|l|}{ Suitability } \\
\hline & & High & Moderate & Low & Nil \\
\hline Openness & Land cover & Alpine & Alpine forest & Forest & Other \\
\hline Openness & Canopy closure & Bare $(0-5 \%)$ & Sparse $(6-25 \%)$ & Moderate $(26-65 \%)$ & Dense $(66-100 \%)$ \\
\hline Remoteness & & $10-80 \mathrm{~km}$ & $81-100 \mathrm{~km} \mathrm{1-9km}$ & $101-500 \mathrm{~km}$ & $>500 \mathrm{~km}$ \\
\hline Road access & & No roads & $\begin{array}{l}\text { Unploughed roads, } \\
\text { logging roads }\end{array}$ & Groomed trails & Other roads \\
\hline \multirow{2}{*}{\multicolumn{2}{|c|}{$\begin{array}{l}\text { Slope } \\
\text { Topographic position }\end{array}$}} & $5^{\circ}-25^{\circ}$ & $26^{\circ}-30^{\circ}$ & $<5^{\circ}$ & $>30^{\circ}$ \\
\hline & Topographic position & Crest, upper slope & Mid slope, low slope & Toe, depression. & - \\
\hline Snow condition & Aspect & $315^{\circ}-45^{\circ}$ (north facing) & $46^{\circ}-134^{\circ}, 226^{\circ}-314^{\circ}$ & $135^{\circ}-225^{\circ}$ (south facing) & - \\
\hline Snow condition & Elevation & $>1800 \mathrm{~m}$ (above treeline) & $\begin{array}{l}1200-1800 \mathrm{~m} \\
\text { (below treeline) }\end{array}$ & $<1200 \mathrm{~m}$ & - \\
\hline
\end{tabular}

Tabelle 2: Modellvariablen für die Geländeeignung für Schneemobilfahren, Geländeeignung für Schneemobilfahren (Quelle: Kliskey, 2000).

Darauffolgend wurden die Gewichtungsfaktoren von den Hauptkomponentenwerten der einzelnen Komponenten für jede Variable abgeleitet. Als letzter Schritt erfolgte die Eignungsüberlagerungskartierung mittels GIS und der Raumkriterien. Das Ergebnis dieser Methode ist eine Kartierung der Erholungseignung, die die räumliche Ausdehnung der Erholungseignung der Landschaft für eine Erholungsnutzergruppe darstellt, nämlich die der Schneemobilfahrer in den North Columbia Mountains von British Columbia.

\subsection{Verfahren für eine komplexe Bewertung der Erholungseignung von Land- schaft und Infrastruktur}

Eine Reihe weiterer Bewertungsverfahren beziehen neben den naturräumlichen, landschaftlichen und Landnutzungskriterien auch die touristische bzw. Freizeitinfrastruktur in die Bestimmung der Erholungseignung ein. So werden Landschaftsbewertungen durch Infrastrukturbewertungen ergänzt oder mit Siedlungsbewertungen kombiniert.

Kiemstedt et al. erweiterten bei ihrer Erholungsbewertung im Sauerland auf diese Weise die Erholungseignung der Landschaft durch eine Bewertung der erholungswirksamen 
Ortsinfrastruktur und durch die Bildung von Ortstypen. Als Zielgruppe wurden Bevölkerungsschichten des Ruhrgebiets und der Rheinschiene mit niedrigem und mittlerem Einkommen bestimmt, deren Ansprüche vom therapeutischen Erholungswert und dem Kostenaufwand abhängen. Im Vorfeld dieser Bewertung wurden landschaftsbezogene Freizeitaktivitäten nach ihrem therapeutischen Wert für die Erholung, nach dem privaten Kostenaufwand der Erholungssuchenden und der relativen Häufigkeit der Beteiligung der Zielgruppe (Arbeiter) an den einzelnen Erholungsaktivitäten, sowie ihrer Bedeutung im Untersuchungsraum beurteilt. Die daraufhin ausgewählten Erholungsaktivitäten wurden nach ihrem räumlichen und funktionalen Zusammenhang zu vier Aktivitätskomplexen zusammengefasst:

- Sommererholung am Wasser (Baden/Schwimmen, Lagern/Spielen, Camping, Angeln/Fischen),

- Sommererholung auf dem Wasser (Rudern/Paddeln, Segeln, Dampferfahrten),

- Sommererholung, gebietsgebunden (Spazierengehen/Wandern, Lagern/Spielen, Radfahren, Besichtigen von Sehenswürdigkeiten, Naturbeobachtung, Bergsteigen/Klettern),

- $\quad$ Erholung im Winter (Skilaufen/Skiwandern, Rodeln, Skiabfahrtslauf, Wandern, Schlittschuhlaufen) (Kiemstedt et al., 1975; S. 34).

Die Erholungsbewertung erfolgt getrennt für die Landschaft (Raumeignung für einzelne Erholungsaktivitäten) und die Ortschaften (Vorhandensein und Ausbaugrad der Erholungseinrichtungen).

Das Bewertungsverfahren zur Erholungseignung der Landschaft für die einzelnen Erholungsaktivitäten wurde in zwei Schritten erarbeitet: Zunächst wurde eine Liste von Landschaftselementen, Erholungseinrichtungen und Infrastrukturanlagen zusammengestellt, die für die ausgewählten Freizeitaktivitäten im Untersuchungsraum Sauerland von Bedeutung sind (Relevanzliste A). Bei einigen Kriterien werden weitere Eigenschaften hinzugenommen wie Gewässergütestufen, Tiefenstufen und Exposition von Gewässern, Eigenschaften von Gewässerrändern, Einzelbäume oder Baumgruppen auf Acker-, Wiesen- und Heideflächen. Hinzu kommt eine Reihe von Störfaktoren, die die Erholung insgesamt oder einzelne Erholungsaktivitäten beeinträchtigen. Ihre Relevanz für die einzelnen Erholungsaktivitäten wurde nach folgenden Stufen bewertet:

1 Notwendige landschaftliche oder infrastrukturelle Voraussetzungen zur Ausübung einer Erholungsaktivität (z.B. Forstwege, beschilderte Wanderwege, Skipiste, Sportbootverleih) als Mindestanspruchsniveau;

2 Verbessernde Kriterien, die die landschaftliche Eignung steigern, aber zur Ausübung nicht unbedingt Voraussetzung sind (z.B. Rastplätze mit Bänken, Cafés), zur Güteeinstufung verwendet;

0 Indifferente Kriterien, die auf die Ausübung der Erholungsaktivität keinen Einfluss haben;

$X$ Restriktive Kriterien, die die Ausübung der Erholungsaktivität erheblich einschränken oder unmöglich machen (z.B. Beeinflussen von Lagern/Spielen durch Emissionen oder Lärm);

$1 / 2$ Kriterien, die über die notwendigen Bedingungen hinaus verbessern wirken (z.B. Größe des Badesees) (vgl. Kiemstedt et al., 1975, S. 40/41).

Die flächenbezogene Bewertung erfolgt rasterbasiert $(2 \mathrm{~km} \times 2 \mathrm{~km}$, für den Komplex Sommererholung am Wasser $500 \mathrm{~m} \times 500 \mathrm{~m}$ ) auf einer regionalen Ebene. Als Datengrundlagen dienten dabei Messtischblätter im Maßstab 1:25 000, Großmaßstabskarten im Maßstab 1: 5 000 oder $1: 10$ 000, eine Reihe weiterer Unterlagen zu verschiedenen Themen sowie zwei Erhebungsbögen (A und B) entsprechend der unterschiedlichen Maßstäbe (vgl. Kiemstedt et 
al., 1975; S. 54-64). Anschließend wurde für jedes Untersuchungsquadrat die Erholungseignung für alle Erholungsaktivitäten durchgeführt, sowie eine Gütebewertung für jene Untersuchungsquadrate vorgenommen, für die das Mindestanspruchsniveau 1- sehr gute Qualität bis 4 - keine besondere Qualität erfüllt ist.

Bei der Bewertung der Orte wurden zum einen Bewertungskriterien festgelegt und zum anderen die Orte hinsichtlich der Wetterabhängigkeit und der jahreszeitlichen Nutzung typisiert. In einem nächsten Schritt wurden jene ortsinfrastrukturellen Kriterien dargestellt, die erfassbar und für die einzelnen Ortstypen (wetterunabhängig/ wetterunabhängig und Erholung im Sommer/Winter/ ganzjährig) von Bedeutung waren. Die Bewertung erfolgte mit dem Erhebungsbogen $\mathrm{C}$ auf der Grundlage verschiedensten Unterlagen. Als Bewertungskriterien wurden die Art der Fremdenunterkünfte, die allgemeine touristische Versorgung, Einrichtungen für bestimmte Erholungsaktivitäten, touristische Sehenswürdigkeiten, die klimatische Höhenlage des Ortes und die Erschließungseinrichtungen für den Erholungsverkehr gewählt. Die Abgrenzung und Klassifizierung der Fremdenverkehrsorte wurde auf der Basis der Einwohner und Gästebetten vorgenommen. Weiter betrachtet wurden nur Ortschaften, bei denen der Quotient aus Gästebetten und Einwohnerzahl mindestens 0,02 beträgt.

Bei Barsch \& Saupe (1994) basiert die Bewertung der landschaftlichen Erholungseignung ebenfalls auf Eigenschaften des Reliefs, der Flächennutzung und von Randeffekten (nach MARKS et al. 1992). Bewertet wurden Mikrochoren oder Nutzflächenmuster auf der Ebene der Landschaftsplanung (Gemeinde) mittels Verflechtungsmatrizen und einer individuellen Bewertung der Basiseinheiten. Die Wertigkeit steigt mit zunehmender Reliefenergie, Vielfalt und/oder Gewässernähe sowie zunehmendem Anteil an Randbereichen von Gehölzen und Gewässern (Barsch \& Saupe, et al. 1994; S. 90). Diese landschaftliche Erholungseignung wird jedoch mit einer Bewertung der Realflächennutzung aus Sicht der Freizeitnutzer und der potenziellen Nutzungsmöglichkeit für verschiedene Freizeitnutzergruppen sowie einer Bewertung der Freizeitinfrastruktur verknüpft. Im Ergebnis dieser komplexen Erholungsbewertung entsteht ein kombinierter Erholungswert, der naturräumliche, landnutzungsbezogene und infrastrukturelle Bewertungen aggregiert (vgl. Abbildung 4). 


\section{Landschaftliche Erholungseignung}

Bewertung naturräumlicher Eigenschaften: Reliefenergie, Vielfalt und/oder Gewässernähe der Pflanzendecke, Randbereiche von Gehölzen und Gewässern

individuelle Bewertung

\section{Potenzieller Nutzungsgrad}

Bewertung der Realflächennutzung: Bedeutung für fünf Nutzergruppen (Zahl der Nutzergruppen)

typbezogen

\section{Nutzerbezogener Attraktivitätswert}

Bewertung der Realflächennutzung: Nutzerpräferenzen (allgemeine Nutzerperspektive - Ergebnisse mehrerer Befragungen)

typbezogen

\section{Freizeitinfrastrukturwert}

Bewertung infrastruktureller Ausstattung: Wander- und Radwege, Rastplätze, Freizeiteinrichtungen in Siedlungen

individuell

\section{Kombinierter Erholungswert}

Kombination von landschaftlicher Erholungseignung, Potenziellem Nutzungsgrad, Nutzerbezogenem Attraktivitätswert, Freizeitinfrastrukturwert

typbezogen und individuell

Abbildung 4: Erholungswert einer Landschaft auf der Ebene der Landschaftsplanung.

Quelle: Eigene Zusammenstellung nach Barsch \& Saupe et al., 1994 (S. 89-91).

Die Erholungsbewertung auf der Ebene der Landschaftsrahmenplanung (Region) verbindet in ähnlicher Weise unterschiedliche Kriterien der Landschafts- und Infrastrukturbewertung (vgl. Abbildung 5). Die Basiseinheiten der Bewertung bilden Funktionsgebiete: Wohngebiet, Gewerbegebiet, Klein- und Streusiedlungen, Verkehrsgebiet, Abbau, technische Ver- und Entsorgung, Landwirtschaft, Forstwirtschaft, Erholungsflächen im Freiraum, sonstige Erholungsflächen, Gewässerflächen, Naturnahe Gebiete, Militärgebiete, Sonderareale (Barsch \& Saupe et al., 1994; Anhang S. 18). Die Bewertungen werden entweder direkt aus der Funktion abgeleitet (Planungsrelevanz Bedeutung der Erholung gegenüber anderen Nutzungen, Abwägungsbedarf bei Nutzungsänderungen) oder aus den Werturteilen von Erholungsuchenden zu Landschaftselementen (Nutzerpräferenzen). Darüber hinaus wird für die Freiräume die landschaftliche Vielfalt, für die Gewässer die Erholungsmöglichkeiten und für die Siedlungen (Knoten, in denen die Bewegungsräume der Erholungsuchenden gebündelt werden) die Freizeitinfrastruktur bewertet. Ergänzt werden diese nutzerbezogenen Bewertungen durch human- bzw. geoökologische Bewertungen der Biologischen Reizarmut und der Nachhaltigen Nutzbarkeit für Erholung (vgl. Barsch \& Saupe et al., 1994; S. 89-96). Eine Kombination der Einzelwertungen wurde auf der regionalen Ebene nicht vorgenommen. 


\section{Ordnung nach Nutzerpräferenzen}

Bewertung der Funktionsgebiete:

Ableitung aus Präferenzen von Landschaftselementen (Befragungen in Havelland und Spreewald)

typbezogen

\section{Ordnung nach Planungsrelevanz}

Bewertung der Funktionsgebiete: Bedeutung für die Erholung gegenüber anderen Nutzungen (Abwägung bei Nutzungsänderungen)

typbezogen

\section{Ordnung nach Freizeitinfrastruktur}

Bewertung infrastruktureller Ausstattung: Spektrum der Freizeitinfrastruktur (Gaststätten, Beherbergung, Freizeiteinrichtungen Punktbewertung)

individuell

\section{Ordnung der Freiräume nach landschaft- licher Vielfalt}

Bewertung (kultur-)landschaftlicher Eigenschaften: Vielfalt bzw. Gliederung der Flächen

individuell

\section{Bioklimatische Reizarmut}

Bewertung von Vegetationseigenschaften: Pflanzenbedeckung, -höhe, -schichtung

typbezogen
Ordnung der Gewässer nach Erholungsmöglichkeiten

Bewertung der Gewässerflächen: Möglichkeiten für Erholungsaktivitäten

individuell

\section{Nachhaltige Nutzbarkeit für Erholung}

Bewertung naturräumlicher Eigenschaften:

Resilienz des Pflanzenbestandes, ökologisch wertvolle Biotope

typbezogen

Abbildung 5: Nutzerbezogene Bewertung des Erholungspotentials auf der Ebene der Landschaftsrahmenplanung

Quelle: Eigene Zusammenstellung nach Barsch \& Saupe et al., 1994 (S. 91-96).

Die von Mönnecke \& Wasem (2005) erarbeitete Anleitung zur Berücksichtigung der Naherholung in der kommunalen Planung des Amtes für Raumplanung im Kanton BaselLandschaft verbindet ebenfalls die landschaftliche Eignung mit Kriterien der Infrastruktur sowie der Erreichbarkeit. Im Fokus steht die landschaftsgebundene, naturverträgliche Erholung im Umfeld von Siedlungsräumen. Naherholung wird dabei als „spezifische Erholungsform, die durch den Orts- und Zeitaspekt gekennzeichnet ist, aufgefasst. Naherholungsgebiete schliessen sich in der Regel an das Siedlungsgebiet an. Sie werden einerseits zur Tageserholung am Abend aufgesucht, andererseits für Wochenenderholung genutzt. Die Erholungs- 
und Freizeitaktivitäten dauern maximal einen Tag, weshalb für das Erreichen des Naherholungsgebietes nicht mehr als 60 - 90 Minuten mit öffentlichen oder privaten Verkehrsmitteln aufgewendet wird." (Mönnecke \& Wasem, 2005; S. 4). Ein Bewertungsverfahren wird nicht explizit dargestellt, die Landschaftseignung wird mit Hilfe einer Checkliste beurteilt, mit der, anhand der Anforderungen der einzelnen Sport- und Freizeitaktivitäten, die landschaftlichen Voraussetzungen überprüft werden können. Natürliche und kulturelle Attraktionen sind dabei lediglich beispielhaft aufgelistet. Die Erreichbarkeit von Naherholungsgebieten wird hingegen eher detailliert durch die Verkehrsmittel, die Aufenthaltsdauer im Erholungsgebiet und die Art und Ausstattung des Erholungsgebietes und damit auch durch die möglichen Erholungsaktivitäten bestimmt (vgl. Abbildung 6).

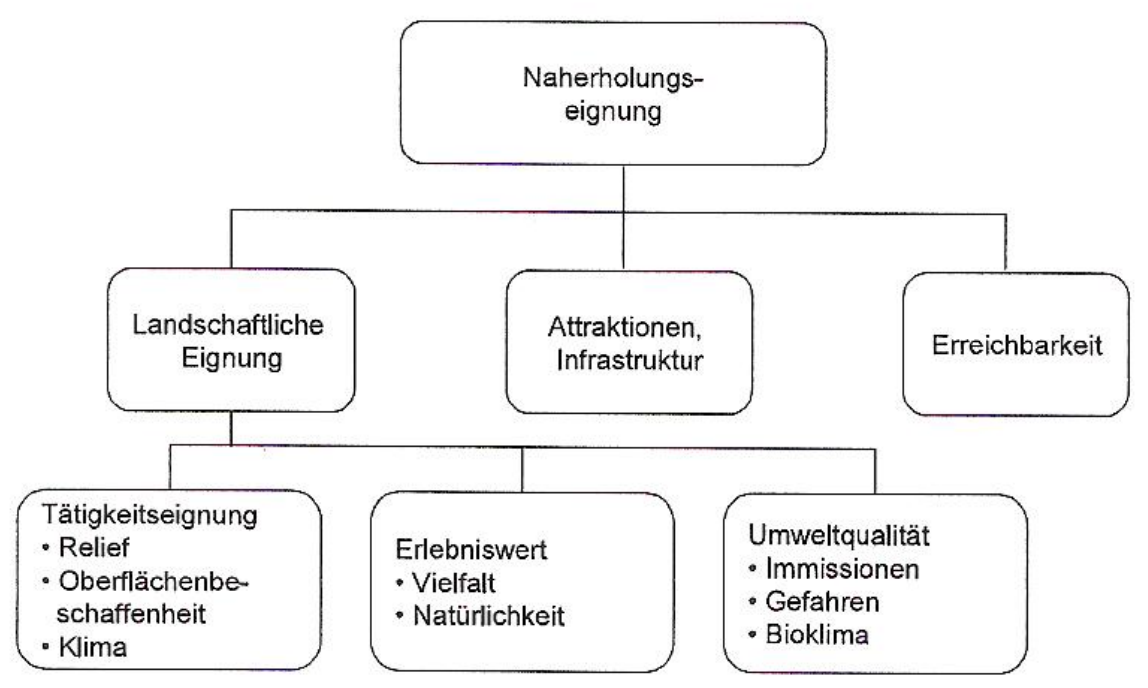

Abbildung 6: Eignungskriterien für die Naherholung.

Quelle: Mönnecke \& Wasem (2005), S. 7, unter Verweis auf HSR \& SRVA 2002: Werkzeugkasten LEK. Eine Arbeitshilfe zum Erarbeiten von Landschaftsentwicklungskonzepten, Rapperswil.

\subsection{Weitere Verfahren mit einem komplexen Bewertungsansatz zur Erholungs- eignung}

Die im Landschaftsplan für den Umlandverband Frankfurt (2000) vorgenommene Beurteilung der Erholungseignung bezieht sich zunächst auf die Bewertung des Erholungspotentials von Bastian (1993). Die Bewertung basiert jedoch nicht auf empirischen Erhebungen oder objektive Analysedaten, sondern sie erfolgt aufgrund der Ortskenntnisse der zuständigen Flächennutzungs- und Landschaftsplaner. Dabei werden visuell abgegrenzte Landschaftsbildeinheiten (z. B. Flur weiträumig, Flur kleinräumig gegliedert, Hangkante, Wald eben, Wald mit Neigung über $5 \%$, Weinberg, Kleingarten, Golfplatz, Fluss/Teich/künstlicher See) nach ihrer Eignung für verschiedene Erholungsarten bewertet. Als Erholungsarten werden

- Wandern, Spazierengehen, Radfahren, (ästhetisches) Landschaftserleben,

- wasserbezogene Erholung an Flüssen, Bächen, Gräben imd Stillgewässern und

- Beobachten von Pflanzen- und Tierwelt 
verwendet. Die Bewertungsmethode nach Bastian wird dabei erweitert, da sie wassergebundener Erholung einen hohen Stellenwert einräumt und die meisten Waldflächen nur eine mäßige bis gute Eignung erreichen. Die Eignungsbewertung wird zunächst für die drei Erholungsarten durchgeführt (1 - fehlende oder sehr geringe Eignung bis 5 sehr gute / hervorragende Eignung) und dann durch eine Addition der Punkte zu einer Gesamteignungsbewertung aggregiert (3-6 ungeeignet bis wenig geeignet, 7-10 mäßig bis gut geeignet, 11-15 sehr gut bis hervorragend geeignet).

Die Komplexität des Bewertungsverfahrens resultiert aus der Einbeziehung unterschiedlicher Arten von Beeinträchtigungen (Lärm, Zerschneidung, Freileitungen, Besuchshäufigkeit, Altlasten). Datengrundlagen bilden Karten (z.B. lärmbelastete Gebiete, Altlasten) und subjektive Einschätzungen. Aus der Kombination von Eignungsbewertung und Beeinträchtigungen wird ein Handlungsbedarf abgeleitet: Bei sehr guter bis hervorragender Eignung existiert kein Handlungsbedarf, wenn es auch keine übermäßige Konzentration von Erholungsuchenden gibt. Handlungsbedarf wird jedoch aufgezeigt, wenn ungeeignete bis wenig geeignete Gebiete durch Erholungsuchende stark frequentiert werden, daraus wird eine Empfehlungskarte abgeleitet. Schließlich werden die unterschiedlichen Landschaftsräume (z.B. Hoher Taunus, Vortaunus, Main-Taunus-Vorland, Kern des Verdichtungsraumes Frankfurt-Offenbach) in inrer Erholungseignung charakterisiert (Anteil der drei Eignungsgruppen) und Empfehlungen aus Sicht der Erholungseignung formuliert (siehe Umlandverband Frankfurt (UVF) 2000b, S. 232-253).

Banzhaf \& Netzband (2000) leisten in ihrem Projekt "Analyses and evaluation of nature space potential in peri- urban spaces using remote sensing data and GIS" das das Naturraumpotential für den suburbanen Raum in der Region zwischen Halle und Leipzig (D) mittels Methoden der Fernerkundung inklusive geografischer Informationssysteme bewertet, ebenfalls einen Beitrag zur Bewertung von Naturraumpotenzial und Erholungseignung. Die Analyse des suburbanen Raums beschäftigt sich mit den Kriterien der Lebensqualität, der Erholung, des Biotopschutzes, der Biodiversität und der Naturästhetik. Auf diese Kriterien hin untersucht das Projekt die Eignung von IRS-1C\&D- Satellitendaten für diverse Planungsanforderungen, wie etwa die Aktualisierung von Landnutzungsplänen, kommunalen Übersichtskarten, Stadtstrukturtypenkarten und Biotopkarten, Oberflächenversiegelungserhebungen und Arbeitskarten für die Landschaftsplanung. Weiters wird das Potential der IRS-1C\&DSatellitendaten untersucht, um die Basis für die aktualisierten, allgemeinen Daten für die Maßstabsebene von 1: 25000 zu schaffen. Das Ergebnis der Studie stellt die Bewertung von Landschaftselementen, Attraktionen und Defiziten in Bezug auf das Naturraumpotential, die Erholungseignung, den Naturschutzaspekt, sowie den Kulturschutzaspekt dar und ermöglicht es, die gewünschten Planungsziele zu kontrollieren und Planungsmängel und potentiale zu erkennen.

\subsection{Zusammenfassung der Kriterien in den Bewertungsverfahren}

Die Erholungseignung stellt sich in der Regel als Ergebnis einer komplexen Bewertung mit unterschiedlichen Komponenten dar:

- Landschaftskomponenten (landschaftliches Potential für Erholung und Freizeitnutzung oder landschaftliche Erholungseignung) und zumeist

- infrastrukturelle Komponenten oder/und kulturhistorische Werte (Landschafts- und Ortsbewertungen) mit

- funktionalen bzw. Lagekomponenten, die die Nutzbarkeit für bestimmte Erholungsformen, Anforderungen von Freizeitaktivitäten sowie Erschließungsmerkmale beinhaltet, sowie 
- Beeinträchtigungen oder Störfaktoren kombiniert.

Dabei lassen sich Bewertungsverfahren, die ausschließlich naturräumliche und Landschaftskriterien beinhalten von solchen unterscheiden, die zusätzlich anthropogene Faktoren, insbesondere die touristische und Freizeitinfrastruktur, die Erreichbarkeit oder/und Störfaktoren betrachten. Bei der Kombination der verschiedenen Faktoren-, Kriterien- und Indikatorengruppen für eine komplexe Bewertung der Erholungseignung weisen die untersuchten $\mathrm{Be}-$ wertungsverfahren eine Reihe von Spezifika auf, die in Tabelle 3 dargestellt sind, wobei GIS gestützte Methoden grau hinterlegt sind. 
Tabelle 3: Spezifika von Bewertungsverfahren zur Erholungseignung.

\begin{tabular}{|c|c|c|c|c|c|}
\hline Kriterien/Indikatoren & $\begin{array}{l}\text { Bewertungsgegen- } \\
\text { stand Raum / Maß- } \\
\text { stabsebene }\end{array}$ & $\begin{array}{l}\text { Räumliche Basis- } \\
\text { einheiten, Karten I } \\
\text { GIS }\end{array}$ & $\begin{array}{l}\text { Freizeitaktivitäten I } \\
\text { Nutzergruppen }\end{array}$ & $\begin{array}{l}\text { Ergebnis, Verfahren, } \\
\text { Aggregation }\end{array}$ & Autor / Quelle \\
\hline $\begin{array}{l}\text { Naturnähe und Ab- } \\
\text { wechslungsreichtum }\end{array}$ & $\begin{array}{l}\text { Landschaftspotential } \\
\text { für naturbezogene Er- } \\
\text { holung (mehrere Zeit- } \\
\text { schnitte), NP-Region } \\
\text { Sächs. Schweiz } \\
\text { (Ackerbau, Wald/Fels) }\end{array}$ & GIS, rasterbasiert & $\begin{array}{l}\text { Spazierengehen, Wan- } \\
\text { dern }\end{array}$ & $\begin{array}{l}\text { Erholungswert, Additi- } \\
\text { on der normierten Pa- } \\
\text { rameterwerte }(0-1)\end{array}$ & $\begin{array}{l}\text { Langzeitmonitoring: } \\
\text { Auswirkungen des } \\
\text { Landschaftswandels } \\
\text { auf die Erholungseig- } \\
\text { nung } \\
\text { WALZ/BERGER } 2004\end{array}$ \\
\hline $\begin{array}{l}\text { Natürlichkeitsgrad, } \\
\text { Gewässer, Diversität } \\
\text { der Nutzungsformen, } \\
\text { Reliefvielfalt }\end{array}$ & $\begin{array}{l}\text { Landschaftstypen (z,B, } \\
\text { technogene/urbane } \\
\text { Gebiete, Agrargebiete, } \\
\text { Wald-Agrar- } \\
\text { Mischgebiete) } \\
\text { Maßstabsebene } \\
\text { 1:50000 }\end{array}$ & Landschaftseinheiten & $\begin{array}{l}\text { Wandern, Spazieren- } \\
\text { gehen, Radfahren, } \\
\text { Landschaftserleben, } \\
\text { wasserbezogene Erho- } \\
\text { lung, Beobachtung der } \\
\text { Pflanzen- und Tierwelt }\end{array}$ & $\begin{array}{l}\text { Delphi-Methode, (Auf- } \\
\text { wand vergleichsweise } \\
\text { gering) }\end{array}$ & $\begin{array}{l}\text { Interaktives Bewer- } \\
\text { tungshandbuch - So- } \\
\text { zioökonomische Be- } \\
\text { wertungsverfahren, } \\
\text { Verfahren von Bastian } \\
(1993)\end{array}$ \\
\hline $\begin{array}{l}\text { Gebietseigenschaften } \\
\text { des Waldareals, Na- } \\
\text { turausstattung, erho- } \\
\text { lungsmindernde Merk- } \\
\text { male, Nachbarschafts- } \\
\text { verhältnis zu Siedlun- } \\
\text { gen und anderen Wald- } \\
\text { flächen }\end{array}$ & $\begin{array}{l}\text { Erholungsfunktion des } \\
\text { Waldes in Naherho- } \\
\text { lungsgebieten ab } 50 \text { ha } \\
\text { Größe } \\
\text { Rand von Frank- } \\
\text { furt/Main, im Hochtau- } \\
\text { nus und im Kaufunger } \\
\text { Wald }\end{array}$ & $\begin{array}{l}\text { Waldflächen, begrenzt } \\
\text { durch Verkehrslinien, } \\
\text { Mindestgröße } 50 \text { ha } \\
\text { (ab } 500 \text { ha Teilung } \\
\text { nach Abteilungen) } \\
\text { Karten }\end{array}$ & keine & $\begin{array}{l}\text { Erholungswert } \\
\text { Ermittlung der Einzel- } \\
\text { werte durch Formeln, } \\
\text { Zuordnung einer } \\
\text { 6stufigen Skala, einfa- } \\
\text { che math. Aggregation } \\
\text { durch arithmet. Mittel, } \\
\text { Produktbildung u.ä. }\end{array}$ & $\begin{array}{l}\text { RUPPERT (1971): Zur } \\
\text { Beurteilung der Erho- } \\
\text { lungsfunktion sied- } \\
\text { lungsnaher Wälder }\end{array}$ \\
\hline $\begin{array}{l}\text { Wald: Offenheit, Abge- } \\
\text { legenheit, Hanglage, } \\
\text { Erreichbarkeit, Topo- } \\
\text { graphie, Schneelage }\end{array}$ & $\begin{array}{l}\text { Erholungseignung von } \\
\text { Wald für Snowmobiling } \\
\text { Maßstabsebene: } \\
\text { 1: } 20000 \\
\text { North Columbia Moun- } \\
\text { tains in British Colum- } \\
\text { bia }\end{array}$ & $\begin{array}{l}\text { digitale topographische } \\
\text { Karten, digitale Wald- } \\
\text { bestandskarten (inkl. } \\
\text { Berücksichtigung von } \\
\text { Wildtierhabitaten) }\end{array}$ & Snowmobiling & $\begin{array}{l}\text { (RTSI)- Eignungsindex } \\
\text { für die Abgrenzung } \\
\text { der räumlichen Aus- } \\
\text { dehnung eines mögli- } \\
\text { chen Erholungsgelän- } \\
\text { des in der } \\
\text { Landschaft }\end{array}$ & $\begin{array}{l}\text { KLISKEY (2000): Rec- } \\
\text { reation terrain suitabil- } \\
\text { ity mapping: a spatially } \\
\text { explicit } \\
\text { methodology for de- } \\
\text { termining recreation } \\
\text { potential for resource } \\
\text { use assessment }\end{array}$ \\
\hline
\end{tabular}

IESP - Towards Integrated Ecological Spatial Planning for the Wienerwald Biosphere Reserve 


\begin{tabular}{|c|c|c|c|c|c|}
\hline Kriterien/Indikatoren & $\begin{array}{l}\text { Bewertungsgegen- } \\
\text { stand Raum / Maß- } \\
\text { stabsebene }\end{array}$ & $\begin{array}{l}\text { Räumliche Basis- } \\
\text { einheiten, Karten I } \\
\text { GIS }\end{array}$ & $\begin{array}{l}\text { Freizeitaktivitäten I } \\
\text { Nutzergruppen }\end{array}$ & $\begin{array}{l}\text { Ergebnis, Verfahren, } \\
\text { Aggregation }\end{array}$ & Autor / Quelle \\
\hline $\begin{array}{l}\text { L: natürliche und infra- } \\
\text { strukturelle Merkmale } \\
\text { (notwendige, verbes- } \\
\text { sernde, indifferente, re- } \\
\text { striktive), } \\
\text { O: infrastrukturelle, so- } \\
\text { ziale und kulturelle } \\
\text { Ausstattung für den } \\
\text { Fremdenverkehr }\end{array}$ & $\begin{array}{l}\text { Erholungseignung der } \\
\text { Landschaft }(\mathrm{L}) \text {, Bewer- } \\
\text { tung der erholungs- } \\
\text { wirksamen Ort- } \\
\text { sinfrastruktur }(\mathrm{O}) \text {, Sau- } \\
\text { erland }\end{array}$ & $\begin{array}{l}\text { L: Untersuchungsquad- } \\
\text { rate } \\
\text { O: Siedlungsbereiche }\end{array}$ & $\begin{array}{l}\text { Bevölkerungsschichten } \\
\text { des Ruhrgebiets und } \\
\text { der Rheinschiene mit } \\
\text { niedrigem und mittle- } \\
\text { rem Einkommen, aus- } \\
\text { gew. Erholungsaktivitä- } \\
\text { ten und Aktivitätskom- } \\
\text { plexe }\end{array}$ & $\begin{array}{l}\text { L: Relevanz der Land- } \\
\text { schafts-/ Infrastruktur- } \\
\text { merkmale, Kriteriensys- } \\
\text { tematik, } \\
\text { Gütebewertung auf den } \\
\text { Aggregationsstufen der } \\
\text { Kriterien } \\
\text { O: Ortstypen, An- } \\
\text { spruchs-niveaus der } \\
\text { Ortstypen }\end{array}$ & $\begin{array}{l}\text { KIEMSTEDT et al. } \\
\text { (1975): Landschafts- } \\
\text { bewertung für Erholung } \\
\text { im Sauerland }\end{array}$ \\
\hline $\begin{array}{l}\text { landschaftliche Eig- } \\
\text { nung, Attraktionen/ Inf- } \\
\text { rastruktur, Erreichbar- } \\
\text { keit }\end{array}$ & $\begin{array}{l}\text { Eignung für die Naher- } \\
\text { holung (landschaftsge- } \\
\text { bundene, naturverträg- } \\
\text { liche Erholung im Um- } \\
\text { feld von Siedlungsräu- } \\
\text { men) } \\
\text { kommunale Planung }\end{array}$ & k.A. & $\begin{array}{l}\text { ausgew. Sport- u. } \\
\text { Freizeitaktivitäten / Mo- } \\
\text { tivgruppen } \\
\text { (z.B. Spazieren, Wan- } \\
\text { dern,, Inlineskaten, } \\
\text { Joggen, Laufen, Wal- } \\
\text { king, Schwimmen, ...) }\end{array}$ & $\begin{array}{l}\text { Checklisten mit Anfor- } \\
\text { derungen der Freizeit- } \\
\text { oder Sportaktivitäten, } \\
\text { Richtwerte }\end{array}$ & $\begin{array}{l}\text { MÖNNECKE/WASEM } \\
\text { 2005: Anleitung zur Be- } \\
\text { rücksichtigung der } \\
\text { Naherholung in der } \\
\text { kommunalen Planung }\end{array}$ \\
\hline $\begin{array}{l}\text { Vielfältigkeitswert: } \\
\text { Wald- und Gewässer- } \\
\text { rand, Relief und Nut- } \\
\text { zungsarten, Klima }\end{array}$ & $\begin{array}{l}\text { Vielfältigkeitswert der } \\
\text { Landschaft, Maßstabs- } \\
\text { ebene unbekannt }\end{array}$ & Karten & keine & $\begin{array}{l}\text { Summe der Vielfältig- } \\
\text { keitswerte multipliziert } \\
\text { mit Klimafaktor (For- } \\
\text { mel) }\end{array}$ & $\begin{array}{l}\text { KIEMSTEDT (1967): } \\
\text { Zur Bewertung natürli- } \\
\text { cher Land- } \\
\text { schaftselemente für die } \\
\text { Planung von Erho- } \\
\text { lungsgebieten }\end{array}$ \\
\hline $\begin{array}{l}\text { Reliefenergie, Land- } \\
\text { schaftsvielfalt. Gewäs- } \\
\text { sernähe, Randberei- } \\
\text { che, nutzerbezogene } \\
\text { Attraktivität (Erholung } \\
\text { allg.), potenzielle Nut- } \\
\text { zungsmöglichkeiten für } \\
\text { bestimmte Nutzergrup- }\end{array}$ & $\begin{array}{l}\text { Erholungswert auf der } \\
\text { Ebene der Land- } \\
\text { schaftsplanung (lokal, } \\
\text { Gemeinde) } \\
\text { Töplitz, - Uetz-Paaren, } \\
\text { Petzow - Ferch }\end{array}$ & $\begin{array}{l}\text { eigenes GIS, } \\
\text { LP: Nutzflächenmuster }\end{array}$ & $\begin{array}{l}\text { teilweise ausgewählte } \\
\text { Freizeitnutzergruppen } \\
\text { (z.B. ältere naturbe- } \\
\text { wusste Ausflügler und } \\
\text { Naherholer, Kurz- u. } \\
\text { Langzeiturlauber, Frei- } \\
\text { zeitwohner u. Dauer- } \\
\text { camper) }\end{array}$ & $\begin{array}{l}\text { Typbezogene und indi- } \\
\text { viduelle Bewertungen } \\
\text { kombinierter Erho- } \\
\text { lungswert, } \\
\text { komplexe Bewertung } \\
\text { über Verflechtungsmat- } \\
\text { rizen, Punktbewertun- } \\
\text { gen und Summationen, }\end{array}$ & $\begin{array}{l}\text { BARSCH/SAUPE } \\
\text { (1994): Bewertung und } \\
\text { Gestaltung der natur- } \\
\text { nahen Landschaft }\end{array}$ \\
\hline
\end{tabular}

IESP - Towards Integrated Ecological Spatial Planning for the Wienerwald Biosphere Reserve 


\begin{tabular}{|c|c|c|c|c|c|}
\hline Kriterien/Indikatoren & $\begin{array}{l}\text { Bewertungsgegen- } \\
\text { stand Raum I Maß- } \\
\text { stabsebene }\end{array}$ & $\begin{array}{l}\text { Räumliche Basis- } \\
\text { einheiten, Karten I } \\
\text { GIS }\end{array}$ & $\begin{array}{l}\text { Freizeitaktivitäten I } \\
\text { Nutzergruppen }\end{array}$ & $\begin{array}{l}\text { Ergebnis, Verfahren, } \\
\text { Aggregation }\end{array}$ & Autor / Quelle \\
\hline \multicolumn{6}{|l|}{$\begin{array}{l}\text { pen, Freizeitinfrastruk- } \\
\text { tur }\end{array}$} \\
\hline $\begin{array}{l}\text { Nutzerpräferenzen, } \\
\text { Planungsrelevanz, } \\
\text { Landschaftsvielfalt, } \\
\text { Freizeitinfrastruktur, } \\
\text { Erholungsmöglichkei- } \\
\text { ten d. Gewässer }\end{array}$ & $\begin{array}{l}\text { nutzerbezogene Be- } \\
\text { wertung des Erho- } \\
\text { lungspotenzials und - } \\
\text { angebots auf der Ebe- } \\
\text { ne der Landschafts- } \\
\text { rahmenplanung (regio- } \\
\text { nal), } \\
\text { Spreewald }\end{array}$ & $\begin{array}{l}\text { eigenes GIS, } \\
\text { Funktionsgebiete (z.B. } \\
\text { Wohnen, Verkehr, } \\
\text { Landwirtschaft, Erho- } \\
\text { lungsflächen Gewäs- } \\
\text { ser, Naturnahe Gebie- } \\
\text { te, Militär) }\end{array}$ & $\begin{array}{l}\text { ausgewählte Freizeit- } \\
\text { nutzer-gruppen (z.B. } \\
\text { ältere naturbewusste } \\
\text { Ausflügler und Na- } \\
\text { herholer, Kurz- u. } \\
\text { Langzeiturlauber, Frei- } \\
\text { zeitwohner) }\end{array}$ & $\begin{array}{l}\text { Typbezogene und indi- } \\
\text { viduelle Bewertungen, } \\
\text { keine Kombination } \\
\text { der ordinalskalierten } \\
\text { Einzelwerte }\end{array}$ & $\begin{array}{l}\text { BARSCH/SAUPE } \\
\text { (1994): Bewertung und } \\
\text { Gestaltung der natur- } \\
\text { nahen Landschaft }\end{array}$ \\
\hline $\begin{array}{l}\text { Ästhetische Bewertung } \\
\text { ländlicher Räume }\end{array}$ & $\begin{array}{l}\text { Landschaftsmerkmale } \\
\text { wie Oberfläche, Ge- } \\
\text { wässer, Vegetation, } \\
\text { Tierwelt, gebaute Um- } \\
\text { welt } \\
\text { Maßstabsebene } \\
1: 25000\end{array}$ & Landschaftseinheiten & $\begin{array}{l}\text { vier Typen von Erho- } \\
\text { lungsuchenden: Natur- } \\
\text { typ, Traditionstyp, Akti- } \\
\text { vitätstyp, Promeniertyp } \\
\text { (Gewichtungsprofilen) }\end{array}$ & $\begin{array}{l}\text { 4stufige Bewertung der } \\
\text { Landschaftskomponen- } \\
\text { ten, Addition zu Ge- } \\
\text { samtpunktzahl je Land- } \\
\text { schaftseinheit und } \\
\text { Klassifizierung }\end{array}$ & $\begin{array}{l}\text { Interaktives Bewer- } \\
\text { tungshandbuch }- \text { So- } \\
\text { zioökonomische Be- } \\
\text { wertungsverfahren } \\
\text { Verfahren von Gros- } \\
\text { jean (1986) }\end{array}$ \\
\hline $\begin{array}{l}\text { Multi- Kriterien Bewer- } \\
\text { tungen des suburbanen } \\
\text { Raums hinsichtlich Le- } \\
\text { bensqualität, Erholung, } \\
\text { Biotopschutz, Biodiver- } \\
\text { sität und Naturästhetik }\end{array}$ & $\begin{array}{l}\text { Landschaftsstruktur- } \\
\text { analyse im suburbanen } \\
\text { Raum, Maßstabsebene } \\
1: 25000 \\
\text { Region zwischen Halle } \\
\text { und Leipzig (D) }\end{array}$ & $\begin{array}{l}\text { IRS-1C\&D Satelliten- } \\
\text { daten für vielfältige } \\
\text { Planungs- } \\
\text { Anforderungen, z. B. } \\
\text { Aktualisierung von } \\
\text { Landnutzungsplänen, } \\
\text { kommunale Über- } \\
\text { sichtskarten, Stadt- } \\
\text { strukturtypenkarten, } \\
\text { Biotopkarten und Ar- } \\
\text { beitskarten für Land- } \\
\text { schaftsplanung }\end{array}$ & keine & $\begin{array}{l}\text { Bewertung von Land- } \\
\text { schaftselementen, Att- } \\
\text { raktionen und Defiziten } \\
\text { Durch die Ergebnisse: } \\
\text { - Kontrolle der ge- } \\
\text { wünschten Planungs- } \\
\text { ziele, } \\
\text { - Erkennen von Pla- } \\
\text { nungsmängel und - } \\
\text { potenzialen }\end{array}$ & $\begin{array}{l}\text { BANZHAF } \\
\text { NETZBAND (2000): } \\
\text { Analyses and evalua- } \\
\text { tion of nature space po- } \\
\text { tential in peri-urban } \\
\text { spaces using remote } \\
\text { sensing data and GIS }\end{array}$ \\
\hline
\end{tabular}

Quelle: Eigene Zusammenstellung. 


\subsubsection{Schlussfolgerungen für die Bewertung der Erholungseignung im Wienerwald}

> Landschaftsbewertungen, die die Erholungseignung beschreiben, allgemein für alle Erholungsuchenden oder Freizeitnutzer bzw. für Freizeitnutzertypen, wie Ausflügler, Naherholer, oder Dauercamper (Barsch \& Saupe, 1994), sind für die Fragestellungen im Projekt ebenso wenig geeignet wie die meisten Bewertungen landschaftlicher Potentiale auf der Basis von Kiemstedt et al. (1975) und Marks et al. (1992).

$>$ Von den Verfahren, die die Landschaft und/oder Infrastruktur im Hinblick auf ausgewählte Freizeitaktivitäten bewerten, liefern lediglich Kiemstedt et al (1975) und Mönnecke \& Wasem (2005) einige Hinweise, wenngleich ein Bezug zu den SIRA nur bei wenigen Aktivitäten hergestellt werden kann - Radfahren, Lagern/Spielen (am Wasser/in Landschaft), Joggen - und die Kriterien teilweise sehr allgemein sind.

$>$ Bei den Bewertungsverfahren kristallisiert sich eine Kombination von landschaftlichem Erholungspotential, freizeitrelevanter Infrastruktur (im IESP überwiegend Wege, Picknickplätze, Hundezonen), Störfaktoren und Lageeigenschaften heraus. Dabei ist zu fragen, ob die Lage in einen kombinierten Eignungswert einbezogen werden soll oder als ein zweites Merkmal zur Landschaftseignung hinzukommt - geeignete Flächen in der Nähe zum Siedlungsraum, geeignete Flächen in größerer Distanz, etc. Zu bedenken ist dabei, das günstig gelegene Erholungsareale häufig auch besser erschlossen sind und ein dichteres Wegenetz aufweisen.

$>$ Die Art- und Weise der Verknüpfung oder Aggregation der Einzelwerte - z. B. Summation, Normierung, Formeln, Aufwertung und Abwertung, Verflechtungsmatrix, schrittweise Aggregation - können Anregungen für IESP liefern.

$>$ Ebenso könnte die Unterscheidung in typbezogene und individuelle Bewertungen (Barsch \& Saupe, 1994) die Bewertung einerseits strukturieren und andererseits erleichtern. Typbezogen erfolgt dabei die Bewertung von Basiseinheiten (z. B. Landnutzung, viell. auch Wegedichte) mittels bestimmter Kriterien (Formeln). Bei einer individuellen Bewertung werden die einzelnen Flächen entsprechend ihres Inventars bewertet, was für den gesamten Wienerwald eher schwierig werden dürfte. 


\subsubsection{Naherholung}

Im Projekt IESP steht vor allem die Freizeitnutzung im Naherholungsbereich im Fokus, da der Biosphärenpark Wienerwald durch seine unmittelbare Nähe zu einer Großstadt ein wichtiges Naherholungsgebiet darstellt.

\subsubsection{Begriffsdefinitionen}

Im Themengebiet der Naherholung gibt es unterschiedliche Begriffsdefinitionen, die sich im Laufe der Zeit gewandelt haben und sich teilweise widersprechen. Im Folgenden wird daher zunächst der Begriff des Tagesausflugsverkehrs erläutert, welcher um einiges unmissverständlicher gebraucht wird als der Begriff Naherholung. Tagesausflugsverkehr bzw. Tagestourismus stehen als Oberbegriff für die meisten später genannten Definitionen für Naherholung.

Das Deutsche Wirtschaftswissenschaftliche Institut für Fremdenverkehr (DWIF) definiert Tagesausflugsverkehr folgendermaßen: „Als Tagesausflug wird jedes Verlassen des Wohnumfeldes bezeichnet, mit dem keine Übernachtung verbunden ist und das

- $\quad$ nicht als Fahrt von oder zur Schule, zum Arbeitsplatz, zur Berufsausübung vorgenommen wird

- $\quad$ nicht als Einkaufsfahrt zur Deckung des täglichen Bedarfs dient und

- nicht einer gewissen Routine oder Regelmäßigkeit unterliegt (z.B. regelmäßige Vereinsaktivitäten im Nachbarort, tägliche Krankenhausbesuche, Arztbesuche, Behördengänge, Gottesdienstbesuche)“ (DWIF, 1995; S.2).

In dieser Definition des Tagesausflugsverkehrs des DWIF und auch in vielen anderen Definitionen in der Literatur der Begriff des Wohnumfeldes genannt wird und Wohnumfelderholung teilweise fälschlicherweise synonym mit Naherholung verwendet wird, bedarf es einer kurzen Erklärung des Begriffs Wohnumfeld(erholung).

Von Kilpper et al. (1985, S. 9) wird das Wohnumfeld folgendermaßen definiert:

„Wohnumfeld könnte man als jenen Lebensbereich bezeichnen, der sich räumlich in Sichtund Fußwegnähe um die Wohnung gruppiert und der durch wesentliche, dem Wohnen zugeordnete Lebensfunktionen mitbestimmt wird. "

Für Drum (1981) beginnt das Wohnumfeld jenseits der Fenster und Türen der Wohnungen und umfasst deren gesamte unmittelbare Umgebung. Hierzu gehören der Hausvorbereich, Vorgärten und Wohngärten, Innen- und Hinterhöfe, gemeinschaftliches Siedlungsgrün, Wege, Plätze und das öffentlich nutzbare Grün.

Nach Lang (1988) umfasst Wohnen mehr als die Summe der Aktivitäten in den eigenen vier Wänden. So steht Wohnen für ein differenziertes Geflecht räumlich - funktionaler Beziehungen mit der gebauten Wohnung als Mittelpunkt, die aber eng mit ihrer direkten Umgebung, dem Wohnumfeld, im Zusammenhang steht. In diesem Wohnumfeld werden Klima und Vegetation unmittelbar erlebt, es werden vielfältige Freizeitaktivitäten ausgeübt und es entstehen Nachbarschaft und soziale Kontakte. Das Bayrische Staatsministerium des Innern (1996) geht durch dessen Gliederung in privat, privat/gemeinschaftlich, halböffentlich/ öffentlich und öffentlich sogar noch detaillierter auf den Begriff des Wohnumfeldes ein, wobei auch 
hier wie schon bei vorangegangenen Definitionen jene Bereiche zum Wohnumfeld zu zählen sind, die direkt an die Wohnung/ das Wohnhaus anschließen.

Als maximale Distanz zur Wohnstätte wird von vielen Autoren ein Radius von $300 \mathrm{~m}$ gesehen, was in etwa einer Fußwegdauer von fünf Minuten entspricht (ARF, 1982; Al-Sahili und Aboul-Ella, 1992; Kaufmann, 2000; Bundesamt für Raumentwicklung ARE, 2006).

Inwieweit sich der Naherholungsraum von dem des Wohnumfeldes unterscheidet, soll nun durch die Definition des Begriffes Naherholung erläutert werden.

Die Naherholung stellt eine bedeutende Variante des Tagesausflugsverkehrs dar. Der Begriff wurde erstmals von Karl Ruppert und Jörg Maier Ende der 1960er Jahre thematisiert und definiert und wird bis heute in zahlreichen Werken verwendet.

Ruppert \& Maier (1969) definieren Naherholung als kurzfristige Erholung in einer relativ geringen Entfernung von der Stadt und mit einer relativ kurzen Dauer des Verkehrsvorgangs. Weiters unterscheiden Ruppert \& Maier (1969) zwischen der innerstädtischen und außerstädtischen Erholung, der Wochenend- oder Feiertagserholung und der stundenweisen Erholung (Ruppert \& Maier, 1969 zitiert in Schnell, 2004).

Ein Begriff, der dem der Naherholung nahe kommt, ist der von Kaspar (1996) eingeführte Begriff des Tagestourismus. So charakterisiert er den Tagestourismus als die Verweildauer von einem Tag an einem Ort. Auch die die Planung und Durchführung einer Tour mit dem Zweck der Entspannung und Erholung, der Bildung und Unterhaltung, sowie der sportlichen Betätigung an einem Tag fallen für Kaspar (1996) unter Tagestourismus.

Auf die Definition der Naherholung von Ruppert \& Maier (1969) und auf die des Tagestourismus von Kaspar (1996) bauen zahlreiche Definitionen auf, beziehungsweise ergänzen diese. Im Folgenden wird eine Auswahl solcher Definitionen vorgestellt:

Schnell, 2004 hat die Definition von Ruppert \& Maier aus dem Jahr 1969 um folgenden Zusatz ergänzt: „[...] Die Naherholung findet außerhalb des unmittelbaren Wohnumfeldes im städtischen Randbereich und im Stadtumland statt und setzt eine Distanzüberwindung zwischen Quell- und Zielgebiet voraus. Diese Distanzüberwindung kann im engeren Wohnumfeld fußläufig, ohne Benutzung von Transportmitteln geschehen, kann innerhalb einer Siedlungsfläche Transportmittel beinhalten und ist außerhalb der Siedlungsfläche unabdingbar mit der Verwendung von Transportmitteln verbunden. Naherholung ist ein außerhäusliches Freizeitverhalten ohne Übernachtung" (Schnell, 2004).

Kurzzeiterholung (Feierabend-, Tages-, und Wochenenderholung) im Nahbereich der Städte und Ballungsräume. Auch Ausflug und Kurzzeiterholung fallen darunter. (Deutsche Gesellschaft für Freizeit, 1986 zitiert in Schnell, 2004).

Im Gegensatz zur Deutschen Gesellschaft für Freizeit grenzt Schnell (2004) den Begriff "Ausflug" von dem der "Naherholung“ ab: So sieht Schnell (2004) Fahrten von sechs und mehr Stunden, bei denen Gemeindegrenzen überschritten werden, als „Ausflug“. Weiters definiert er einen Ausflug als eine komplexe Freizeitaktivität, die eine Ortsveränderung anzeigt und zahlreiche Einzelaktivitäten wie etwa Spazierengehen enthält. Ebenso wie Naherholung beinhaltet sie jedoch nur Tätigkeiten, die nicht mehr als einen Tag in Anspruch nehmen und dementsprechend auch nicht mit einer Übernachtung verbunden sind. (Schnell, 2004)

Nach Billion \& Flückiger (1979) umfasst der Begriff „touristische Naherholung“ die Feierabend-, sowie die Tages- und die Wochenenderholung außerhalb des unmittelbaren Wohnumfeldes im städtischen Randbereich und im Stadtumland. Dementsprechend ist sie auf die Dauer von wenigen Stunden bis zu maximal einem Tag begrenzt und umfasst damit keine Übernachtung. Ein weiteres Kriterium für touristische Naherholung stellt für Billion \& Flückinger (1979) eine Distanzüberwindung zwischen Quell- und Zielgebiet dar (Billion \& Flückiger, 1979 zitiert in Schnell, 2004). 
Kulinat \& Steinecke (1984) definieren Naherholung als außerhäusliches Freizeitverhalten ohne Übernachtung, wobei zwischen Erholungsraum im Wohnumfeld, innerörtlichem Erholungsraum und außerörtlichem Erholungsraum unterschieden wird (Kulinat \& Steinecke, 1984 zitiert in Schnell, 2004) Naherholung findet zwar außerhalb, aber in recht geringer Entfernung vom Stadtgebiet bei einer verhältnismäßig kurzen Aufenthaltsdauer statt und betrifft vornehmlich an Freiraum gebundene Freizeitaktivitäten (vgl. Eberle, 1980 und Kulinat, 1980 zitiert in Weiss, 1991).

Im Diercke Wörterbuch: „Allgemeine Geografie“ (Leser, 2005) wird jener Teil des Freizeitverhaltens außer Haus als Naherholung gesehen, der sich von einer mehrstündigen Erholung bis hin zu einem Wochenendausflug erstrecken kann. Der Begriff „nah“ ist bei dieser Definition stärker im zeitlichen, als im räumlichen Zusammenhang zu verstehen, da die Naherholung bestimmter sozialer Gruppen zum Teil in beträchtlicher Entfernung zum Wohnort stattfindet. Eine regelmäßige Naherholung ist besonders bei Einwohnern von großstädtischen Verdichtungsräumen sehr hoch. Dementsprechend versteht Leser (2005) ein Naherholungsgebiet als den Teil einer Landschaft, der durch seine natürliche Attraktivität und/oder seine Infrastrukturausstattung besonders als Zielgebiet für den Naherholungsverkehr geeignet ist und dementsprechend auch stark frequentiert wird. Leser (2005) definiert auch den Begriff des Naherholungsraumes, wobei er darunter jenen Raum versteht, der regelmäßig von der Bevölkerung oder bestimmter sozialer Gruppen zur Naherholung aufgesucht wird. Die Grenzen eines Naherholungsraumes ergeben sich dabei durch die Reichweiten des Naherholungsverkehrs, also durch jenen Teil des Freizeit- und Erholungsverkehrs, der durch Fahrten aufgrund von Naherholung bedingt ist. Dementsprechend ist der Naherholungsverkehr besonders stark auf das Wochenende konzentriert und ist hauptsächlich zwischen den großstädtischen Verdichtungsräumen und den innen nahegelegenen Naherholungsgebieten angesiedelt. Zur Reichweite im Naherholungsverkehr zählt im Speziellen die Lage attraktiver Naherholungsgebiete, was bewirkt, dass ein städtischer Naherholungsraum je nach den Zielen im Sommer- und Winterausflugsverkehrs unterschiedlich ausgebildet ist (Leser, 2005).

Das Naherholungsverhalten wird nach Leser (2005) durch die Art und Weise, auf die Naherholung ausgeübt wird, charakterisiert. Dieses Verhalten ist sowohl regional und je nach sozialen Gruppen unterschiedlich, sowie von Modeströmungen, Prestigegesichtspunkten und dem Angebot an Naherholungseinrichtungen in erreichbarer Entfernung vom Wohnort abhängig (Leser, 2005).

Nach Wolf \& Appel-Kummer umfasst Naherholung einen Tages-, Altags- oder Wochenendausflug (bis zu drei Übernachtungen), der in der Nähe von der Wohnung bzw. dem Wohnort stattfindet. In der Nähe des Wohnortes bedeutet, dass das Naherholungsgebiet in eineinhalb bis zwei Stunden erreicht wird, wobei der Erholungsradius aufgrund der vorhandenen guten Verkehrsinfrastruktur und der damit einhergehenden, steigenden Mobilität stetig anwächst. Eine Kopplung von Zeit und Entfernung ist jedoch notwendig, um Naherholung von Kurzurlauben, wie etwa Städtereisen, zu unterscheiden.

\subsubsection{Räumliche und zeitliche Dimension der Naherholung}

Bereits 1970 erkannten Maier \& Ruppert, dass die räumliche Komponente zur Begriffsdefinition an Bedeutung abnimmt, da durch die wachsende Mobilisierung bei gleich bleibender Zeit immer größere Entfernungen zurückgelegt werden (Maier \& Ruppert 1970 zitiert in Wolf \& Appel-Kummer, 2009). 
Räumliche Dimension der Naherholung:

Wie die genannten Definitionen zeigen, beginnt Naherholung mit dem Verlassen des Wohnumfeldes, also mindestens mit dem Verlassen der eigenen Wohnung. Naherholung kann auf intralokaler, interlokaler und intraregionaler Ebene stattfinden:

- Intralokale Naherholung bezeichnet Naherholung innerhalb der eigenen Stadt bis maximal an die Stadtgrenze

- Interlokale Naherholung findet zwischen zwei Städten bzw. außerhalb der eigenen Stadtgrenze statt und

- Intraregionale Naherholung findet innerhalb einer Region statt (Cermak, 2008).

Die in Kauf genommene Entfernung, um in das Erholungsgebiet zu gelangen, ist stark geprägt vom Mobilitätsverhalten, das durch subjektive und objektive Komponenten bestimmt wird. Die sozialen Komponenten beziehen sich auf das Mobilitätsbedürfnis und die Motive des Einzelnen, während die objektiven Komponenten durch die Verkehrsinfrastruktur, die Wohnsituation, das Einkommen und die Siedlungsstruktur geprägt sind (Amt der NÖ Landesregierung \& NÖ Landesakademie, 2003).

Aus vielen Befragungen und Untersuchungen ist bekannt, dass das meistgenutzte Verkehrsmittel zu Freizeitzwecken das Auto ist (vgl. Maschke, 2006, Opaschowski, 1999). Eine in Niederösterreich durchgeführte Mobilitätsbefragung $(n=9000)$ bestätigt ebenso den hohen Anteil an Autofahrten: $68 \%$ aller mobilen Personen sind mit dem Auto unterwegs, wobei der Großteil der Wege zu Freizeitzwecken zurückgelegt werden. $24 \%$ der sonntäglichen Wege werden in Niederösterreich zu Fuß zurückgelegt, $5 \%$ mit dem Rad und $3 \%$ wählen öffentliche Verkehrsmittel (Amt der NÖ Landesregierung \& NÖ Landesakademie, 2003).

In einer Studie von Stettler wurde festgestellt, dass die mittleren Wegdistanzen für die Ausübung von Sport stark von der Sportart abhängig sind und im Durchschnitt 12,2 Kilometer für eine Wegrichtung betragen. Die geringsten Mobilitätswerte weisen Jogger $(4,6 \mathrm{~km})$ und Reiter (9,3 km) auf, während Wanderer durchschnittlich 25 Kilometer zurücklegen (Stettler, 1997 zitiert in Opaschowski, 1999). Knoflacher hat erhoben, dass unter der Woche eine Anfahrtszeit von maximal 15 Minuten in Kauf genommen wird, was einem Aktionsradius von 10 Kilometern entspricht, wenn man mit dem PKW unterwegs ist (Knoflacher, 2002 zitiert in: Wolf \& Appel-Kummer, 2009).

\section{Zeitliche Dimension der Naherholung}

Opaschowski sieht die 60-Minuten-Distanz als ein wesentliches Merkmal für die Wahl des Freizeitziels an, das heißt ein Fahrziel muss in der Regel innerhalb von 60 Minuten erreichbar sein. Bei Tagesausflügen darf die Anfahrt länger sein: Die durchschnittlich akzeptierte Fahrzeit liegt bei 1,1 Stunden, bei Familien mit Kindern gar bei 1,3 Stunden. Das heißt somit, dass ein Ziel bis zu 100 Kilometer weit entfernt sein kann. Je attraktiver das Freizeitziel ist, desto größer ist die Bereitschaft lange Wegstrecken zurückzulegen (Opaschowski, 1999). In der niederösterreichischen Mobilitätsbefragung wurde festgestellt, dass die durchschnittlichen Wege, welche die Niederösterreicher am Sonntag zu Freizeitzwecken zurücklegen, 36 Minuten bzw. 21 Kilometer betragen. Im Vergleich dazu werden unter der Woche durchschnittlich 27 Minuten bzw. 12 Kilometer zu Freizeitzwecken zurückgelegt (Amt der NÖ Landesregierung \& NÖ Landesakademie, 2003). 


\subsubsection{Einflussfaktoren zur Teilnahme an Naherholung}

Mittels einer Reihe von Faktoren wird bestimmt, wie oft potenzielle Naherholungsräume aufgesucht bzw. Freizeitaktivitäten unternommen werden. Dazu zählen demografische Merkmale, sozio-ökonomische Merkmale, das Mobilitätsverhalten, die Wohnsituation, die verfügbare Freizeit und die verfügbare Information über Freizeitmöglichkeiten (vgl. Opaschowski, 1997). In Bezug auf die demografischen und sozioökonomischen Merkmale spielen Geschlecht, Beruf, Ausbildungsstand, Wohnort, Religion, Einkommen und Familienstand eine Rolle.

Im Durchschnitt hat jede Person fünf Stunden und zehn Minuten pro Tag für Freizeit zur Verfügung. In Abhängigkeit der obengenannten Einflussfaktoren können Unterschiede im Freizeitbudget von plus/minus ein bis zwei Stunden festgestellt werden (Prahl, 2002). Bislang lässt sich außerdem ein stabiler Trend in Richtung einer Ausdehnung der täglich verfügbaren Freizeit beobachten (Wolf \& Appel-Kummer, 2009).

Die Wahl des Naherholungsgebiets ist stark vom Mobilitätsverhalten geprägt, das wiederum durch subjektive und objektive Komponenten bestimmt wird. Die sozialen Komponenten beziehen sich auf das Mobilitätsbedürfnis und die Motive des Einzelnen, während die objektiven Komponenten durch die Verkehrsinfrastruktur, die Wohnsituation, das Einkommen und die Siedlungsstruktur geprägt sind.

Die Auswahl des Naherholungsgebiets wird ebenso von einer Reihe von Faktoren geprägt. Zu diesen zählen die Ausstattung des Gebiets, die Qualität, die Standortfaktoren, das Image, die Erreichbarkeit, die Vertrautheit, Transportmöglichkeiten und die Wahl der Freizeitaktivität. Die Wahl der Freizeitaktivität wiederum wird geprägt von der verfügbaren Zeit, dem Wetter und der Jahreszeit, der Kenntnis über die Freizeitaktivität, die Gruppenstruktur und die Wahl des Naherholungsgebiets (vgl. Cermak, 2008)

\subsubsection{Naherholung in der Natur}

Erholung in naturnahen Landschaften erfordert eine geeignete Ausstattung dieser Räume, um Aktivitäten auszuüben. Insbesondere land- und forstwirtschaftliche Flächen stellen großflächige, zusammenhängende Räume für die Naherholung dar (Wolf \& Appel-Kummer, 2009). So wird für die Naherholung ein Waldanteil zwischen 50 und $70 \%$ aus Sicht der Erholungsnutzenden als besonders geeignet empfunden (Hinterberger et al., 2002 zitiert in Wolf \& Appel-Kummer, 2009). Insbesondere im Nahbereich von Ballungsräumen werden Erholungsgebiete gut angenommen, wenn die zur Verfügung gestellte Freizeitinfrastruktur (Markierungen, Gasthäuser, ...) die Nutzungsvielfalt erhöht. Da heute das persönliche Wohlbefinden im Mittelpunkt der Naherholung steht, ist die Nachfrage nach Erholungsangeboten in der Natur sehr hoch und wird vermutlich auch noch anwachsen. Die Grenzen von Naherholung, Kurzurlaub und Urlaub werden fließender werden, weshalb Naherholungsräume eine bisher nicht gekannte Bedeutung bekommen werden (Wolf \& Appel-Kummer, 2009).

Eine Studie aus Deutschland zeigt, dass rund $67 \%$ der Bevölkerung ihre Freizeit im Freien verbringen und nahe gelegene Erholungsräume nutzen. Wie bereits aufgezeigt, ist der Aktionsradius der Erholungssuchenden relativ weit; im Rahmen von Naherholungsaktivitäten unter der Woche werden jedoch bevorzugt Flächen und Räume in Wohnnähe aufgesucht (Feige, 1996 zitiert in Heinze \& Kill, 1997 zitiert in Wolf \& Appel-Kummer, 2009). 


\subsubsection{Erwartungen an den Naherholungsraum}

Ergebnisse von Hinterberger et al. (2002) weisen darauf hin, dass vor allem die Vielfältigkeit einer Landschaft wahrgenommen wird und sich aus unterschiedlichen Landschaftstypen unterschiedliche Erwartungen ableiten lassen. Aus Sicht der Erholungssuchenden gibt es eine Reihe von Faktoren, die für die Attraktivität von Erholungsräumen relevant sind, wobei wiederum für die Ausgestaltung von Erholungsräumen urbaner und ländlicher Räume Faktoren unterschiedlicher Relevanz festgestellt werden können.

Bezüglich der Ausgestaltung werden folgende Anforderungen gestellt:

- Abwechslungsreiches Nebeneinander von Wald, Acker, Wiesen und Weiden.

- Vorhandensein nutzbarer Freiräume für Spiel, Sport und Erholung.

- Ein ausgebautes Spiel-, Wander- und Radwegenetz, sowie infrastrukturelle Angebote für die unterschiedlichen Natursportarten.

- Vorhandensein charakteristischer, typischer und eigenartiger Landschaftselemente (Felsgruppen, Einzelbäume,..).

- Das Klima sollte unbelastet sein und geringe Extreme aufweisen.

- Klimatische Besonderheiten wie Schneesicherheit sind günstig.

- Das Wasser (Bäche und Flüsse sowie Teiche und Seen) soll sauber, zugänglich und nutzbar sein.

- Die Vegetationsform soll natürlich sein. Unkultiviertes Land und natürliche Sukzession sollen zugelassen werden.

- Landschaftsgliedernde Elemente sollen vorhanden sein.

- Das Gelände sollte ein Relief haben und Besonderheiten (Bodendenkmale) aufweisen.

- Das Landschaftsbild soll abwechslungsreich sein und unterschiedliche Wildpflanzen, Kulturpflanzen, Nutztiere und Wildtiere beherbergen (Wolf \& Appel-Kummer, 2009).

Abwechslungsreiche Wälder wie z.B. Mischwald werden gegenüber Monokulturen eindeutig bevorzugt. Laub- und Nadelbäume in unterschiedlichen Altersklassen, Höhen und Gruppierungen sollten vorkommen, sowie eine gut ausgebildete Kraut- und Strauchschicht vorhanden sein. Einsehbarkeit und Durchlichtung des Waldes sind den Erholungsnutzenden ebenfalls wichtig, dunkle und dichte Bereiche werden im Allgemeinen eher gemieden. Außerdem sind jahreszeitliche Unterschiede in der Bevorzugung des Ortes für die Erholung, die sich aus dem jahreszeitenabhängigen Erscheinungsbild der Vegetation ergeben, feststellbar. Im Frühjahr sind vor allem die Randzonen des Waldes attraktiv, während im Sommer und im Winter das Waldesinnere bevorzugt werden (Wolf \& Appel-Kummer, 2009).

Der Abwechslungsreichtum und die Attraktivität der Landschaft werden zusätzlich durch das Vorhandensein von Lichtungen, lichten Waldinnenrändern, alten und starkholzreichen Beständen, Aussichtspunkten und den Wechsel von Wald und Offenland gesteigert.

Neben diesen allgemeinen Ausstattungsmerkmalen sind zielgruppenorientierte Angebote und spezielle landschaftliche Voraussetzungen erforderlich. Zielgruppenspezifische Strukturen stellen z.B. die bevorzugte Wegeoberfläche (Erde, Gras, Kies oder Asphalt) dar (Wolf \& Appel-Kummer, 2009). 


\subsubsection{6 (Nah)erholung als Thema in der Raumplanung}

Freizeitansprüche in Natur und Landschaft führen zu einer intensiven Nutzung der Naturund Erholungsräume, die sich in der Nähe von Ballungsgebieten befinden. Die Bereitstellung von Angeboten und Freiräumen in der Stadt ist in erster Linie eine öffentliche Aufgabe, die hauptsächlich von Regionen und Gemeinden durchgeführt wird. Um diesen Anforderungen Rechnung zu tragen, stehen zahlreiche Instrumente bzw. Gesetze zur Verfügung, diese Gebiete entsprechend auszuweisen.

Einerseits gibt es entsprechende Regelungen in den einzelnen Naturschutzgesetzen und andererseits sind durch Raumordnungsgesetze und -instrumente die Möglichkeiten gegeben, planend einzugreifen.

Durch die Europäische Union wird das Europäische Raumentwicklungskonzept (EUREK) formuliert. In diesem geht es vor allem darum, eine ausgewogene und nachhaltige Entwicklung innerhalb der EU zu gewährleisten, wobei die Sicherung von Kulturlandschaften und der Natur wichtige Themen sind. In Bezug auf die Erholung ist das EUREK zur Umsetzung von erholungsrelevanten Inhalten als wichtig zu erachten.

Da in Österreich die Raumordnung und -planung von Bund, Ländern, Städten und Gemeinden wahrgenommen wird, stehen dementsprechend auch verschieden Planungsinstrumente zur Verfügung:

- Österreichisches Raumentwicklungskonzept (Bundesebene)

- Landesraumordnungsprogramme/-konzepte, Raumordnungs-Sachprogramme für das gesamte Landesgebiet (Landesebene)

- Regionale Raumordnungsprogramme, Regionale Raumordnungs-Sachprogramme (Regionalebene)

- Örtliches Entwicklungskonzept, Flächenwidmungsplanung, Bebauungsplanung (Gemeindeebene)

Das Österreichische Raumentwicklungskonzept (ÖREK) wird von der Österreichischen Raumentwicklungskonferenz erstellt und hat eine gemeinsame Strategie für die räumliche Entwicklung in Österreich zum Ziel. Im ÖREK 2001 wird die Wichtigkeit der Naherholung thematisiert, wobei jedoch nicht zwischen Wohnumfeld- und Naherholung differenziert wird:

„Grünräume, Freiräume vor allem in wohnnaher Lage sind wichtige Naherholungsgebiete bzw. Orte für die naturgebundene Freizeitgestaltung. Die wohnnahe Lage mit fußläufiger Erreichbarkeit ist insbesondere für Kinder, Jugendliche und SeniorInnen wichtig."

Unter „2.6.3 Ziele und Strategien“ wird weiters auf die Relevanz einer Lenkung der zukünftigen Entwicklung der Naherholung eingegangen: „(446) Zentren, Wohn- und Betriebsgebiete, sowie Flächen für den ökologischen Ausgleich und die Naherholung sind differenziert, aber gleichzeitig im stadtregionalen Zusammenhang zu entwickeln. (470) Die bestehenden Naherholungsflächen innerhalb der dichter besiedelten Kernräume sind - soweit überhaupt vorhanden - zum Teil zu gering dimensioniert und stark übernutzt. Die Städte und Gemeinden kaufen jedoch kaum Flächen zur Einrichtung oder Erweiterung von integrierten Naherholungsflächen auf, da sie zu teuer sind bzw. ökonomisch nicht in Wert gesetzt werden können. Zu überlegen sind neue Wege der Finanzierung von stadtregionalen Naherholungsflächen."

Besonders relevant für diese Arbeit ist jedoch folgendes Ziel des ÖREK 2001: „(531) Ein besondere Herausforderung stellt die Entwicklung der Landschaft als Träger der freiraumorientierten Naherholung und des Tourismus dar. Freizeit und Urlaub bedeuten zunehmend Aktivität und Abenteuer, individuelles Erleben, Selbsterfahrung und Konsum. Die 
herkömmliche „passive“ Begegnung mit der Natur genügt nicht mehr, Landschaft wird zur veränderbaren Kulisse für inszenierte Freizeit- und Sportaktivitäten. Dabei müssen jedoch die Grenzen des Verträglichen erkannt und akzeptiert werden. Wenn die Inszenierung des Erholungsraumes die prägenden Grundelemente der Landschaft in Frage stellt oder wenn eine "industrialisierte“ Tourismuswirtschaft in nicht mehr standortgerechte Formen mündet, dann sind diese Grenzen erreicht."

Auf Landesebene betreffen das Landesentwicklungskonzept Niederösterreich, sowie das Stadtentwicklungskonzept Wien den Biosphärenpark Wienerwald.

Das Stadtentwicklungskonzept Wien (2005) sieht im Bereich des südlichen Wiener Beckens ein Forcieren des Wegenetzes für Naherholung als Ziel: „Naherholung: vor allem das südliche Wiener Becken bietet attraktive Voraussetzungen für die Naherholung, vor allem zum Reiten und Radfahren. Erste Maßnahmen für anzulegende Wegenetze wurden vom „Verein NÖ-Wien, Gemeinsame Erholungsräume“ initiiert und müssen weiter fortgesetzt werden."

Explizit den Wienerwald betreffend besteht ein Ziel des Stadtentwicklungskonzeptes Wien (2005) darin, sowohl den Naturschutz, als auch die Erholungsnutzung zu erhalten: „1987 wurde von den Bundesländern Wien und Niederösterreich die Wienerwalddeklaration verabschiedet, welche 2002 überarbeitet und erneuert wurde. Ziel dieser politischen Willenserklärung ist es, Schutz- und Entwicklungsziele für die Region so aufeinander abzustimmen, dass der Wienerwald als Natur- und Erholungsraum erhalten bleibt, gleichzeitig aber eine nachhaltige wirtschaftliche Entwicklung ermöglicht wird."

Im Landesentwicklungskonzept Niederösterreich (2001) wird auf eine für diese Arbeit wichtige Begebenheit, eingegangen, die das Tourismusverhalten der Wienerwaldbesucher und somit die Naherholung betrifft: „Niederösterreich ist ein bedeutendes Freizeit- und Naherholungsland. Es ist allerdings nicht das „klassische“ Tourismusland, sondern vielmehr ein Land „zum Wohnen“ und eine beliebte Destination für Ausflüge, Naherholung, Kurzurlaube und Zweitwohnsitze."

Ebenso wie im Stadtentwicklungskonzept Wien (2005), wird auch im Landesentwicklungskonzept Niederösterreich (2001) die Wichtigkeit des Wienerwaldes bezüglich Naturschutz und Erholungswirkung hervorgehoben: „Eine besondere Bedeutung besitzen dabei der Wienerwald sowie die Aulandschaften östlich von Wien. Die Funktion des Wienerwaldes als ökologischer Ausgleichsraum und Naherholungsgebiet soll durch eine besonders sparsame Inanspruchnahme des Bodens für bauliche Nutzungen sowie durch Raumordnungs- und Umweltschutzmaßnahmen sichergestellt werden (vgl. Bioshärenpark). “ 


\subsubsection{Wildtiere Störungsökologie}

\subsubsection{Allgemein}

Eine ökologische Störung wird nach Rykiel (1985) als Grund für eine (temporäre oder permanente) Veränderung in einem vorher spezifizierten System definiert. Dabei wirkt der Grund als biotische(r) oder abiotische( $r$ ) physikalische Kraft, Überträger oder Prozess. Die Einteilung der Störung erfolgt anhand der Parameter

- Typ

- Häufigkeit

- Intensität

in die Kategorien:

- „destruction“: Vernichtung von Biomasse

- „discomposition“: Auslöschen, verringern, hinzufügen oder ausweiten einzelner Populationen

- „interference“: Verhinderung von Austauschprozessen

- „suppression“: Verhinderung einer natürlichen Störung

Als indirekt messbare Größe in diesem Zusammenhang ist Stress, als physiologische Folgeerscheinung eines Individuums aufgrund einer Störung zu nennen, wiederum im Vergleich zu einem vorher definierten Ausgangszustand.

Ein für Wildtiere zusätzlich wichtiger Faktor wurde bei Christensen (1988) genannt: die Vorhersehbarkeit von Ereignissen. Seltene unerwartete Ereignisse verursachen markantere Reaktionen als regelmäßig wiederkehrende. Damit eng verbunden ist die Stressbelastung der Organismen durch eine Störung. Bei regelmäßig erfolgenden und von ihrer Intensität her geringen Störungen kann die sogenannte „Habituation“ zum Tragen kommen und die Auswirkungen auf die Tiere abmildern.

Habituation (Gewöhnung) bezeichnen Ingold \& Hüppop (2005) als „Abnahme der Reaktion gegenüber einem bestimmten Reiz". Damit soll unnötiger Energieverlust vermieden werden, der entsteht, wenn das Tier auf ein und denselben Reiz stets dieselbe Reaktion in derselben Intensität zeigen würde. Als Voraussetzung nennen die Autoren eine räumliche und zeitliche Konstanz (Regelmäßigkeit) des Reizes sowie eine gewisse Häufigkeit und vor allem Ungefährlichkeit. Die Reaktion der Tiere auf Freizeitaktivitäten ähnelt dem Feindvermeidungsverhalten gegenüber anderen Tieren.

Sichern, im Rahmen des Feindvermeidungsverhaltens läuft nicht immer nach demselben Schema ab. Je nach Jagdstrategie der involvierten Beutegreifer gibt es Arten, die in regelmäßigeren Abständen sichern und Arten, die in möglichst unregelmäßigen Zeitintervallen sichern, um Beutegreifern weniger Möglichkeiten zu bieten ihre Attacke zu planen. Die Grenzen verlaufen fließend und werden durch Gruppengrößen und die Lichtverhältnisse beeinflusst. Weitere Einflussgrößen, wie z.B. Sättigungsgrad sind wahrscheinlich, aber noch nicht untersucht worden [Beauchamp, 2006].

Die Reaktionen auf Störungen erfolgen in ihrem Ausmaß je nach Tierart unterschiedlich (Hernandez \& Laundre, 2005; Miller et al., 2001). Eine gesonderte Betrachtung der betroffenen Arten ist also von Nöten, da selbst innerhalb von Artgruppen, je nach Einnischung unterschiedliche Reaktionen zu erwarten sind (Ellenberg et al., 2006). 
Ein weiterer wichtiger Aspekt ist die Fluchtdistanz. Sie gibt an ab welcher Entfernung zur wahrgenommene Gefahr ein Individuum flieht, da es energietechnisch völlig sinnlos wäre bei jeder Wahrnehmung sofort zu fliehen (Cooper \& Frederick, 2007; Cooper, 2005; DeBoer et al., 2004; EASON et al., 2006; Geist et al., 2005; Gotanda et al., 2009; Manor \& Saltz, 2005).

\subsubsection{Säugetiere}

Die Literatur zu durch Menschen initiierte Fluchtreaktionen bei Säugetieren wurde von Stankowich (2008) weitgehend zusammengefasst. Er kam zu dem Ergebnis, dass für Säugetiere folgende Aspekte wichtig sind:

- das Verhalten der Personen, besonders wenn sie zu Fuß unterwegs sind und in direktem Weg auf die Tiere zusteuern;

- in welchem Habitat die Störung erfolgt: so werden Störungen in offenen Landschaftsteilen mit ausgeprägteren Fluchtreaktionen beantwortet;

- die Gruppenzusammensetzung nach Sozialklassen: weibliche Stücke mit Jungtieren zeigen deutlichere Fluchtreaktionen als eine Gruppe mit ausschliesslich adulten Tieren;

- Bejagung der Population: bei Bejagung zeigten die Tiere ausgeprägtere Fluchtreaktionen.

Generell ist zu sagen, dass die Reaktion auf einen Störreiz von der inm zugeschriebenen Gefahr abhängt, wodurch beispielsweise sehr laute Reize, wie Sprengarbeiten eine im Vergleich geringere Reaktion hervorrufen, als ein sich näherndes menschliches Wesen. Zu ähnlichen Ergebnissen kommt Reimoser (2012). Sie zeigt, dass sowohl Rotwild als auch Rehwild stärker auf optische als auf akustische Reize reagiert, wobei Gewehrschüsse eine Sonderstellung einnehmen. Das Ausmaß und die Art der Reaktion unterscheiden sich jedoch bei den untersuchten Arten, sowohl in der physiologischen als auch ihrer verhaltenstechnischen Ausprägung, in Abhängigkeit von den saisonalen und diurnalen Aktivitätsrhythmen. So reagiert Rehwild kurz und auffällig auf Störungen, wogegen die Reaktionen von Rotwild weniger augenscheinlich ausfallen, dafür aber länger nachwirken.

Zum Thema Reaktionen auf Störungen aus der Luft zeigte sich, dass höher fliegende Objekte einen geringeren Einfluss ausübten als tief fliegende. Tendenziell werden Menschen als gefährlicher wahrgenommen als Hunde. Zumindest wenn man die Reaktionen der Tierre als Maßstab heranzieht.

Anhand mehrerer Huftierarten hat Caro (1994) eine besondere Strategie zur Feindvermeidung untersucht. Nach dem Entdecken zeigen die Tiere energieaufwändige Bewegungsmuster die dem Angreifer klarmachen sollen, dass eine Attacke reine Energieverschwendung darstellt, da aufgrund der Distanz und / oder der körperlichen Verfassung des Beutetieres ein Fehlschlag vorprogrammiert ist. Im Zusammenhang mit Erholungssuchenden könnte der vermehrte Energieaufwand im Zuge eines solchen Verhaltens problematisch werden, wenn es durch wiederholte Annäherung von Menschen ausgelöst wird.

Tourismus kann sowohl positive als auch negative Einflüsse auf Wildtierpopulationen haben George \& Crooks (2006).

Besucherausschluss funktioniert nur in einem geringen Maß, wenn das betreffende Gebiet nahe bei Wohngebieten liegt. Die Bewohner der umgrenzenden Siedlungen halten sich nicht alle an das Betretungsverbot (selbst Zäune werden überwunden) und nutzen mehr oder weniger die gesamte Fläche. Problematisch sind diesbezüglich freilaufende Hunde. Einschrän- 
ken lassen sich diese Personen offenbar nur durch natürliche Barrieren, wie Kanäle, Teiche oder dichte Vegetation (Antos et al., 2007). Barja et al. (2007) beschreiben die Stressbelastung bei Marderartigen durch Erholungsnutzung. Dabei war die Belastung während der Reproduktionszeit am höchsten. Ähnliche Zusammenhänge werden auch für andere Arten beschrieben.

Beale \& Monaghan (2004) untersuchten die Hypothese, dass gerade die Individuen mit der relativ besten Kondition die markantesten Reaktionen auf Störeinflüsse zeigen. Umgekehrt sind Individuen mit schlechterer Kondition in höherem Maß gezwungen ihr Feindvermeidungsverhalten dem Nahrungserwerb unterzuordnen. Sie werden demnach länger in ihrer augenblicklichen Tätigkeit verharren, oder länger an diesem Ort verweilen, sofern es keine, zumindest gleichwertig ausgestattete Alternative gibt. Das kann zum Beispiel in suboptimalen Habitaten der Fall sein. Tatsächlich zeigte die (in diesem Fall bei Vögeln) mit 22\% ihres Tagesbedarfs an Nahrung unterstützte Gruppe von Steinwälzern (Arenaria interpres) ein ausgeprägteres Sicherungsverhalten, flog im Falle einer Störung früher davon und entfernte sich weiter von der Störquelle. Die Konsequenz ist, dass durch alleiniges Verlassen auf die Messung von Fluchtdistanzen, ohne den Lebensraum und die Kondition der Tiere mit in Betracht zu ziehen, die Gegebenheiten vor Ort falsch eingeschätzt werden. Die Ergebnisse von Blumstein (2006) unterstützen diese These. Sie liefern Hinweise auf weitere Parameter, die die Fluchtbereitschaft einzelner Arten voraussagen, wie Körpergröße, das Alter in dem sie sich zum ersten aktiv an der Reproduktion beteiligen („age at first reproduction“), Ernährung und Lebensweise (sozial oder solitär).

\subsubsection{Vögel}

Bolduc \& Guillemette (2003) liefern unter anderem eine Zusammenfassung aus der Literatur der relevanten Faktoren für den Ausfall von Gelegen. Das sind Aufgaben der Brut und Prädation. Beide hängen zusammen und ihre Wahrscheinlichkeit ändert sich mit dem Entwicklungsstadium in dem sich das Gelege befindet. Je später der Zeitpunkt ist, desto größere Risiken sind die Elterntiere bereit in Kauf zu nehmen, da der Energieaufwand ein neues Gelege zu diesem Entwicklungspunkt zu bringen mit jedem Tag steigt. Dementsprechend ist auch der Einfluss durch menschliche Störung unterschiedlich in seiner Wirkung, je nachdem wann er erfolgt. Weitere Einflussgrößen auf das Ausmaß der Störung sind Nesttarnung, Prädatorendichte, Einzel- oder Koloniebrüter und elterliches Feindabwehrverhalten. Die von innen untersuchten Eiderenten (Somateria mollissima) hatten die wenigsten Verluste, wenn die Annäherungsexperimente in einer späten Brutentwicklungsphase begannen. Die dabei vorkommenden Brutverluste dürften auf opportunistische Prädatoren zurückzuführen sein (in diesem Fall hauptsächlich die in demselben Gebiet nistenden Möwen). Die Möwen nutzten die Chancen, die sich ergaben, nachdem die Eiderenten nach einer Störung kurzfristig das Nest verlassen hatten. Der Einfluss durch Erholungssuchende könnte geringer sein als durch Wissenschafter und Daunensammler, da erstere nur in seltenen Fällen die gesamte Kolonie abgehen. Obwohl Daunensammler oft jegliche weitere Nutzung einer Kolonie zu unterbinden versuchen, kann selbst ihr alleiniges und relativ seltenes Auftreten massive Folgen haben, da sie normalerweise die gesamte Kolonie nutzen. Dies ist wiederum in den frühen Phasen des Brutgeschäfts am problematischsten.

Anderson \& Keith (1980) untersuchten den Einfluss von verschiedenen Landnutzern (Erholungssuchende, Fischer, Wissenschafter etc.) auf Brutkolonien von Pelikanen (Pelecanus occidentalis californicus) und Herrmannsmöwen (Larus heermanni). Dabei kann die Unterscheidung zwischen dem Einfluss von Wissenschaft, Tourismus oder landwirtschaftlicher Nutzung schwierig sein (Bolduc \& Guillemette, 2003). Durch Störung wurde das intra- und 
interartliche Verhalten negativ beeinflusst. Angedachte Maßnahmen können von einer Totalsperre für Besucher bis hin zu eingeschränkten Besuchszeiten reichen.

Bartgeier (Gypaetus barbatus) wurden in ihrem Brutverhalten besonders durch laute Geräusche gestört. Dabei spielte je nach Art der Störung die Distanz zur Störquelle eine Rolle, wie stark die Tiere reagierten. Aufgrund ihres alpinen Lebensraumes wirkten akustische Reize, durch Echobildung und Verstärkung der Resonanz weiter als in der Ebene (Arroyo \& Razin, 2006).

Bei Burger (1981) hängt die Intensität der Störung von der Geschwindigkeit der ausgeübten Tätigkeit ab. Demnach sind Joggen und Rasenmähen als schnelle Tätigkeiten problematischer als langsam erfolgendes Bird-Watching oder Muscheln sammeln. Die Reaktionen verliefen je nach Artengruppe unterschiedlich und reichten von Wiederaufsuchen der ursprünglichen Aufenthaltsorte bei Möwen und Seeschwalben bis hin zu kompletten Ortwechseln bei Reihern und Küstenbewohnern.

Die Ergebnisse von Pearce-Higgins et al. (2007) zeigen eine geringe Störung von bodenbrütenden Goldregenpfeifern (Pluvialis apricaria) durch Freizeitnutzer die sich entlang von Linien bewegen. Die Tiere waren selbst bei einer Zunahme der Besucherzahlen in der Lage ihre Brut erfolgreich zu beenden.

Peters \& Otis (2007) berichten sogar über Zusammenhänge mit sich kurzfristig ändernden Einflussgrößen wie Windgeschwindigkeit und Lufttemperatur, die einen Einfluss auf die Störungsempfindlichkeit der Tiere haben. 


\subsubsection{Konflikte und Konfliktpotenziale}

\subsubsection{Konflikttheorie}

Aus sozialwissenschaftlicher Sicht bestehen Konflikte aus Subjekten, d.h. den Konfliktparteien, die sie austragen, und mindestens einem Objekt, dem Konfliktgegenstand (MEYER 1997). Konflikte sind damit nicht nur sachliche Gegensätze, sondern Auseinandersetzungen zwischen mindestens zwei Personen bzw. Parteien. Anders als bei einem Problem kann die Lösung eines Konflikts daher nicht einseitig erfolgen, sondern nur durch das Agieren beider / aller Konfliktparteien. Die Vorstufe zur Konfliktaustragung ist häufig ein Konfliktpotenzial, aus dem ein Konflikt entstehen kann, aber nicht zwangsläufig entstehen muss. Ein Konfliktpotenzial wird aus den (noch) ruhenden Konfliktparteien und dem Konfliktobjekt bzw. -gegenstand gebildet (vgl. Berkel, 1997; Meyer, 1997). Ein Potential für einen Konflikt kann sich demnach zum einen aus den Konfliktparteien und den Beziehungen zwischen ihnen ergeben. Sie resultieren beispielweise aus Ängsten und Missverständnissen, Informations-, Kommunikations- und Partizipationsdefiziten. Zum anderen ist der Konfliktgegenstand durch Gegensätze oder Spannungsfelder charakterisiert, die schließlich zu den Streitpunkten führen. Bei Raumnutzungskonflikten, wie sie in der vorliegenden Arbeit zwischen dem Wildtiermanagement und der Freizeitnutzung untersucht werden, spielen raumstrukturelle bzw. räumliche Konfliktpotenziale eine zentrale Rolle. Ihre Analyse kann nicht nur dazu beitragen, Konflikte zu lösen, sondern diese bereits im Vorfeld zu vermeiden oder zumindest eine Eskalation zu verhindern (vgl. Ziener 2003 und 2005).

Raumnutzungskonflikte entstehen, wenn entweder eine bereits bestehende Nutzung durch eine andere beeinträchtigt oder gefährdet wird oder wenn unvereinbare Nutzungsansprüche auf dieselbe Fläche bzw. denselben Raum zugreifen. Als Nutzungsansprüche werden im Projekt die Ansprüche der Freizeitnutzer im Zusammenhang mit der Ausübung bestimmter Freizeitaktivitäten (Mountainbiking, Joggen, Aktivitäten mit Hund, Picknicken, Geocaching, Ballonfahren) in einem bestimmten Gebiet angesehen. Diese resultieren aus aktivitätsspezifischen landschaftlichen Präferenzen, Distanzen und Routen sowie zeitlichen Aspekten (vgl. Kapitel 3.2.2). Ebenso können aber auch die Schutzmaßnahmen und Regelungen, die im Rahmen des Wildtiermanagements festgelegt oder angestrebt werden, als Raumnutzungsansprüche verstanden werden. Bei der Sicherung oder Entwicklung von Lebensräumen ist von den spezifischen Anforderungen der einzelnen Wildtierarten (Rotwild, Schwarzwild, Schwarzstorch, Auerwild, Ziesel) an ihren Lebensraum und der Sensibilität gegenüber anthropogenen Störungen auszugehen (vgl. Kapitel 2.2.3 und Kapitel 3.2.1). Raumnutzungskonflikte gibt es auch innerhalb der Freizeitnutzung zwischen verschiedenen Freizeitnutzergruppen und innerhalb des Wildtiermanagements aufgrund der unterschiedlichen Lebensraumansprüche der zu schützenden Wildtierarten oder konkurrierender Ziele für ein Gebiet. Diese können auch die Konflikte zwischen Wildtiermanagement und Freizeitnutzung beeinflussen. Die Komplexität einer Konfliktsituationen wird nicht zuletzt dadurch erhöht, dass neben dem Wildtiermanagement und den Freizeitnutzern verschiedene andere Interessengruppen in den Konflikt involviert sind, insbesondere die jeweiligen Grundeigentümer, bei jagdbaren Tierarten die Jagd, Forstwirtschaft und Landwirtschaft, der Tourismus, die Gemeinden und das Biosphärenpark-Management.

Hinter den eindeutig zu verortenden Raumnutzungskonflikten stehen häufig weiter reichende Interessen- und Zielkonflikte. Grundlegende Schutzinteressen stehen dabei zumeist unterschiedlichen Nutzungsinteressen gegenüber. Die zunehmende Ausdehnung der Freizeitaktivitäten in die Abend- und Nachtstunden kollidiert beispielsweise mit dem Bestreben, gerade diese für die Tiere wichtigen Zeiten der Nahrungsaufnahme von Störungen frei zu halten. Interessen- und Zielkonflikte reichen in ihren Anfängen sehr lange zurück. Sie sind prinzipiell nicht lösbar und können allenfalls gemildert oder entschärft werden. Interessen- und Ziel- 
konflikte werden allerdings auch nicht ständig thematisiert, sondern nur sporadisch ausgetragen, z.B. bei der Erarbeitung von Planungsdokumenten, Gesetzen oder Verordnungen. Sie äußern sich vielmehr indirekt bei der Austragung von Raumnutzungskonflikten. Die Bewältigung von Raumnutzungskonflikten wirkt sich somit positiv auf die Interessenkonflikte aus. Nicht zuletzt haben auch die Konfliktaustragung und -lösung zur Entwicklung von Umweltbewusstsein und der Bereitschaft zu einer naturverträglichen Ausübung von Freizeitaktivitäten („Goldene Regeln“), veränderten Strategien im Bereich der Nutzungsrestriktionen und Besucherlenkung sowie neuen Kommunikations- und Kooperationsformen geführt.

Wie die Konflikte bestehen auch raumstrukturelle Konfliktpotenziale aus divergierenden Elementen wie unvereinbaren Schutz- und Nutzungsansprüchen. Sie beschreiben objektive Eigenschaften der Landschaft und ihrer Nutzung, objektivierte Landschaftsbewertungen oder verallgemeinerte Nutzungsansprüche, unabhängig von der subjektiven Wahrnehmung und Bewertung durch die Konfliktbeteiligten. Ihre Erfassung trägt zu einer sachlicheren Beurteilung des Konflikts bei. Raumstrukturelle Konfliktpotenziale zwischen Naturschutz und Erholungs- bzw. touristischer Nutzung lassen sich in drei Dimensionen darstellen:

- Eine Landschaft wird sowohl als schutzwürdig als auch als attraktiv für Erholung und Freizeitgestaltung bewertet (allgemeine Sicht).

- Es besteht ein ungünstiges Verhältnis zwischen der Sensibilität einer Landschaft und der Intensität ihrer Nutzung für Erholung und Freizeitgestaltung (ökologische Sicht).

- Raumnutzungsansprüche von Erholungsuchenden und Freizeitnutzern stehen Restriktionen des Naturschutzes gegenüber (Nutzersicht)

(vgl. Ziener 2003, S. 66-67).

Im vorliegenden Projekt werden die raumstrukturellen Konfliktpotenziale zwischen dem Wildtiermanagement und der Freizeitnutzung im Rahmen der zweiten Dimension bestimmt. Die Störanfälligkeit / Störungssensibilität der Wildtierarten und ihrer Lebensräume wird der Intensität der Freizeitnutzung gegenübergestellt. Raum-zeitliche Aspekte spielen dabei ebenso eine Rolle wie die Art und Weise der Ausübung einer Freizeitaktivität und das Verhalten der Wildtierarten bei Störungen. Im Bereich des Konfliktmanagements ist dagegen auch die Nutzersicht von Bedeutung. Nicht selten führen Nutzungsrestriktionen und Reglementierungen erst zur Entstehung oder Eskalation eines Konflikts.

Die konkrete Verortung raumstruktureller Konfliktpotenziale führt zu potenziellen Konflikträumen. Diese stellen zunächst unabhängig von der Austragung eines Konflikts Problemräume dar, in denen es zu Konflikten kommen kann. Entsprechend der landschaftlichen Gegebenheiten und der Nutzungsmuster können potenzielle Konflikträume kleinflächig (z.B. Picknickbereich, Kletterfelsen) oder linienhaft in Form eines Korridors beiderseits von Straßen und Wegen, aber auch großräumiger ausgeprägt sein. Dabei ist die Größe des potenziellen Konfliktraums immer im Zusammenhang zur Stärke des Konfliktpotenzials zu sehen. Einerseits verweist ein ausgedehnter potenzieller Konfliktraum auf die regionale Bedeutung des Konfliktpotenzials, anderseits kann auch kleinflächig ein großes Konfliktpotenzial entstehen, wenn etwa bedrohte Tierarten oder besondere Lebensräume betroffen sind.

Die Konfliktaustragung und Konfliktbehandlung stellen einen Prozess dar. Dieser Konfliktprozess wird von Berkel (1997) in einem grundlegenden Modell beschrieben, das sich auch auf Konflikte zwischen Wildtiermanagement und Freizeitnutzung anwenden lässt (vgl. Abbildung 7). Ein Konflikt wird dabei als Interaktionsprozess charakterisiert, der durch die wechselseitigen, aufeinander bezogenen Handlungen der Konfliktparteien entsteht. Aktionen der einen Seite bedingen jeweils Reaktionen der anderen Seite. Den Ausgangspunkt bildet ein Konfliktpotenzial bzw. ein latenter Konflikt. Die Konfliktaustragung beginnt in der Regel mit 
einem auslösenden Ereignis und endet mit einem zeitweiligen oder dauerhaften Konfliktergebnis. Raumnutzungskonflikte zwischen Schutz und Nutzung entstehen zum einen dadurch, dass sich ein bereits bestehendes Konfliktpotenzial objektiv verschärft (z.B. Landschaftsschäden, zunehmender Landschaftsverbrauch, Trendsportarten) oder subjektiv anders wahrgenommen und bewertet wird (z. B. durch ein gestiegenes Umweltbewusstsein). Zum anderen werden Konflikte durch Freizeitprojekte oder durch Schutzmaßnahmen mit entsprechenden Nutzungsrestriktionen initiiert. Anders als bei der Lösung von Aufgaben oder Problemen sind die Handlungen der am Konflikt Beteiligten immer auf die Gegenseite bzw. deren Handlungen bezogen. Dazu gehören unter anderem Informations- und Streitgespräche, Diskussionsrunden, Moderations- und Mediationsverfahren, Pressemitteilungen, Gutachten, Konzepte. Eine zentrale Eigenschaft der Interaktionen in einem Konflikt ist die Reziprozität, die den Konfliktparteien eine wechselseitige Beziehung mit entsprechenden Gegenleistungen sichert, beispielsweise bei der Festlegung von Rechten und Pflichten für beide Seiten. Umgekehrt kann bei einem Mangel an Aufmerksamkeit, Anerkennung und Zuwendung oder einem als feindselig interpretierten Verhalten der Gegenseite eine negative Reziprozität (Vergeltung) zur Verschärfung (Eskalation) des Konflikts führen. Das Reziprozitätsprinzip spielt insbesondere bei Konflikten um Nutzungsrestriktionen und Nutzungsrechte eine Rolle (vgl. Ziener, 2005). So wurde in Konflikten zwischen Freizeitsport- und Naturschutzverbänden gelegentlich angemahnt, dass nicht nur die Umweltverträglichkeit von Freizeitnutzungen, sondern auch die Sozialverträglichkeit von Unterschutzstellungen zu berücksichtigen ist (vgl. Hammerich, Müller \& Schaffrath, 1995).

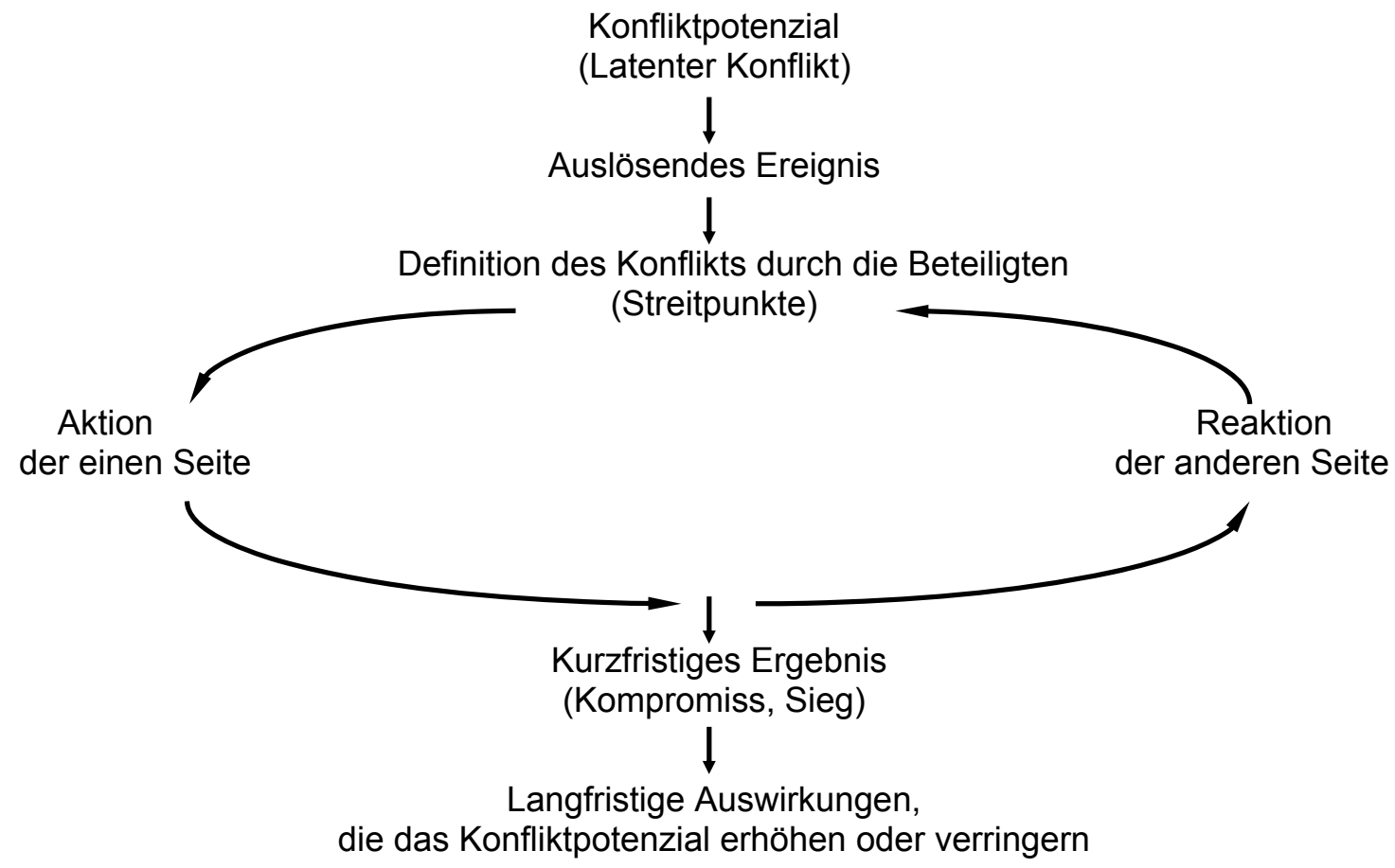

Abbildung 7: Konflikt als Prozess (nach Berkel).

Quelle: BERKEL 1997, S. 40, verändert.

Die Konfliktbewältigung, -beilegung oder -lösung bildet aus dieser Sicht einen integralen Bestandteil des Konfliktprozesses, der sofort mit der Konfliktaustragung beginnt. Die Konfliktlösung ist somit nicht das Ergebnis des Konflikts, sie stellt vielmehr selbst einen Prozess dar. 
Eine endgültige Konfliktlösung, die den Konflikt vollständig beseitigt, kann dabei häufig nicht erreicht werden, weil ein Teil der Konfliktpotenziale weiter bestehen bleiben. Der Konflikt ist vielmehr in den Griff zu bekommen. Dieses Aktiv-Werden in einem Konflikt wird mit Begriffen wie Konfliktbehandlung oder Konflikthandhabung charakterisiert. Situationsbedingt bestehen diese aus unterschiedlichen Interventionsmaßnahmen wie Konfliktlösung, Konfliktreduktion, Konfliktregelung, Konfliktmanagement und Konfliktvermeidung (vgl. Berkel, 1984; Glasl, 1999). Das Konfliktergebnis stellt einen gewissen Abschluss für den Konfliktprozess dar und kann sehr unterschiedlich sein. In Raumnutzungskonflikten reicht das Spektrum von gesetzlichen oder vertraglich fixierten Regelungen und gerichtlichen Entscheidungen bis zu gemeinsamen Nutzungskonzepten sowie informellen Absprachen und Vereinbarungen. Die Qualität des Konfliktergebnisses wird nicht durch die Verbindlichkeit der Regelungen, sondern durch deren Akzeptanz durch die Konfliktparteien bestimmt. Die getroffenen Kompromisse und Regelungen können zeitweilig, vorläufig oder dauerhaft sein. In komplexen Konfliktsituationen haben Regelungen häufig den Charakter von Teillösungen oder Etappensiegen. Bei einer unbefriedigenden oder unzureichenden Konfliktlösung kann der Konflikt wiederum in einen latenten Konflikt übergehen. Die Konfliktwirkungen sind weitaus vielfältiger (vgl. Ziener, 2005). Sie reichen von den direkten Wirkungen auf die Konfliktparteien sowie auf unbeteiligte Zuschauer bis zu den eher indirekten Auswirkungen auf die Landschaft, die Freizeitnutzung, das Wildtiermanagement oder die Region.

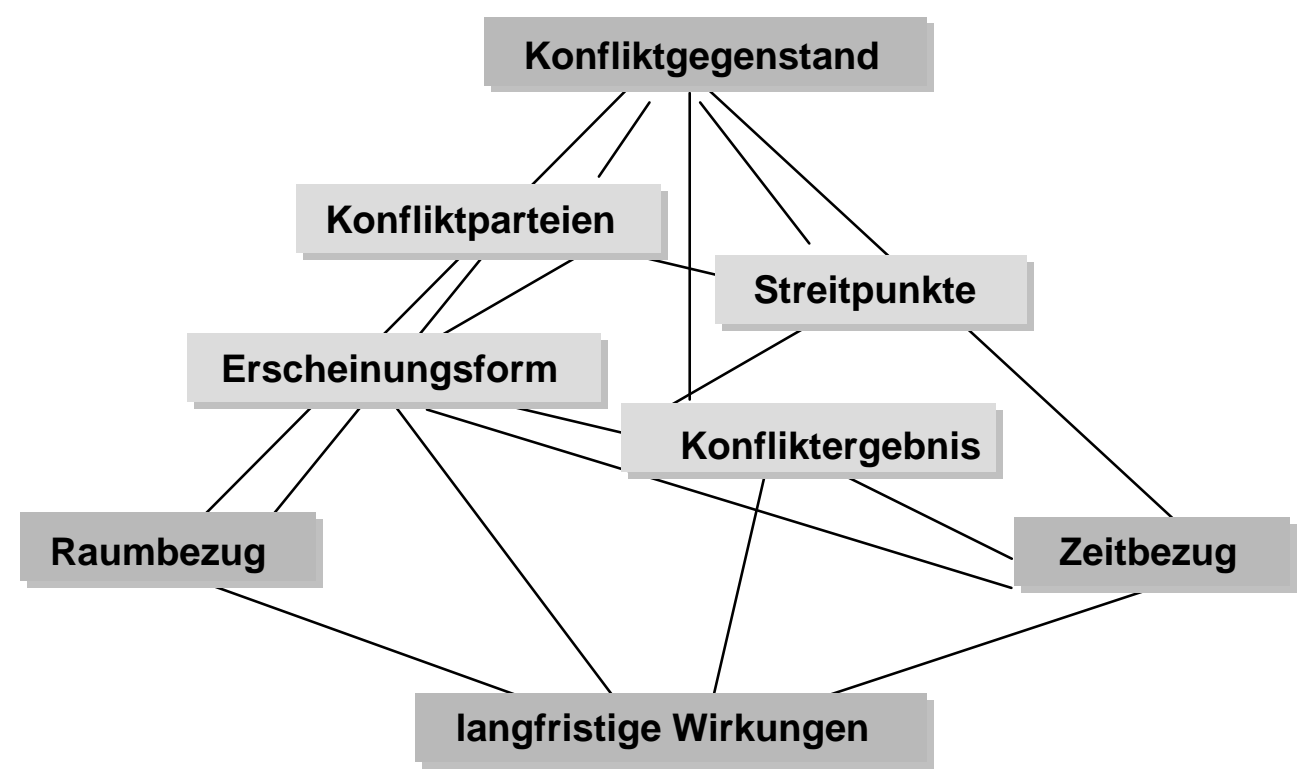

Abbildung 8: Konfliktstruktur [Quelle: Ziener, 2003; S. 81, verändert]

Neben dem Konfliktprozess bildet die Konfliktstruktur die Grundlage für eine detaillierte Konfliktanalyse. Basierend auf Berkel, der in seiner Konfliktdiagnose fünf Merkmale eines Konfliktes verknüpft - Streitpunkte, Konfliktparteien, Erscheinungsform, Verlauf und Ergebnis (Konflikt als Struktur, vgl. Berkel, 1997) - ist die Konfliktstruktur von Raumnutzungskonflikten etwas zu modifizieren (siehe Abbildung 8). Die Strukturelemente der Konfliktaustragung werden durch den Konfliktgegenstand sowie den Raum- und Zeitbezug als äußerem Rahmen ergänzt. Während die Streitpunkte der Konfliktparteien entscheidend sind, wenn es darum geht, Ansatzpunkte für eine Konfliktbewältigung zu finden, stellt der objektivierte Konfliktgegenstand die Verbindung zum Konfliktpotenzial und damit zu den Landschafts- und Nutzungseigenschaften her. Aus dieser Sicht wurden auch die langfristigen Wirkungen dem Konfliktrahmen zugeordnet, wenngleich diese natürlich auch die Konfliktparteien und die Be- 
ziehungen zwischen innen betreffen. Der Raumbezug eines Konflikts ergibt sich zum einen aus der Verortung des Konfliktgegenstands (z. B. Nutzungskonkurrenzen) und zum anderen aus dem räumlichen Wirkungsbereich der Konfliktparteien (z.B. Gemeinde, Region, Biosphärenpark), so dass auch Interessen- und Zielkonflikte einen gewissen Raumbezug haben. Der Zeitbezug umfasst die Dauer des Konfliktprozesses vom auslösenden Ereignis bis zu einem zeitweiligen oder endgültigen Ergebnis, drückt sich aber auch in einem Wechsel von Eskalations- und Ruhephasen (latenter Konflikt) aus. Die Erscheinungsform des Konflikts beinhaltet auch Maßnahmen der Konfliktbehandlung und -bewältigung (vgl. Ziener, 2005).

Ein Teil der Konflikte zwischen Wildtiermanagement und Freizeitnutzung stellt komplexe Konfliktsituationen dar. Als komplexe Konfliktsituationen können Sachverhalte charakterisiert werden, in denen sich mehrere Konflikte und Konfliktfelder bündeln und wechselseitig beeinflussen. Komplexe Konfliktsituationen sind durch eine große Zahl von Beteiligten und eine Vielfalt von Interessen und Ansprüchen gekennzeichnet, so dass für ihre Bewältigung eine umfassende Konfliktanalyse und komplexe Lösungsansätze notwendig sind. (vgl. Ziener, 2003, S. 96). Im Wienerwald entstehen komplexe Konfliktsituationen beispielsweise, wenn Konflikte zwischen Wildtiermanagement und Freizeitnutzung mit Konflikten zwischen verschiedenen Freizeitnutzergruppen, zwischen Freizeitnutzung und Jagd oder zwischen Wildtiermanagement und Siedlungsentwicklung verknüpft sind und somit eine Reihe weiterer Interessengruppen involviert sind. Dabei spielen neben Raumnutzungskonflikten häufig auch Interessen- und Zielkonflikte eine Rolle. Konfliktlösungsansätze sollten daher bei den unterschiedlichen Interessen und Zielen der Konfliktbeteiligten ansetzen, die Komplexität verdeutlichen und vor allem gemeinsame Interessen aufzeigen, die Kooperationen und Partnerschaften ermöglichen. Wechselwirkungen von Konflikten können darüber hinaus auch auf den Konflikterfahrungen der Konfliktparteien beruhen. Das betrifft sowohl die grundlegenden Einstellungen zu Konflikten und die individuellen Herangehensweisen an deren Bewältigung als auch die konkreten Erfahrungen mit den anderen Konfliktparteien aus früheren Konfliktprozessen. Probleme bei der Konfliktlösung sind daher nicht selten bereits auf frühere Konflikte zurückzuführen. Umgekehrt können aber auch positive Erfahrungen bei der Konfliktbehandlung zu konstruktiven Konfliktstrategien führen.

\subsubsection{Beispiele für die Anwendung Multi-Kriterien-gestützter Bewertungsverfahren zur Konfliktpotenzialanalyse}

Mit einer für Österreich eher fremden Landschaft beschäftigen sich Claudet et al. (2010). So stehen bei diesen die Fische und Algen im Cerbere-Banyuls Natural Marine Reserve (französisches Mittelmeer), einem MPA (Multiple-Use Marine Protected Area) und der Einfluss von Schnorchlern auf dieses im Mittelpunkt der Untersuchung. Dennoch stellt dieses Projekt einen interessanten Ansatz für das IESP Projekt dar, da sich Claudet et al. (2010) mit der Landschaft, dem Schutz der Biodiversität und Nutzergruppen beschäftigen. So werden MPAs dazu herangezogen, marine Ressourcen hinsichtlich einer Zuteilung des Raumes an die unterschiedlichen Nutzer und bezüglich der Vermeidung von Konflikten bei gleichzeitigem Schutz der Biodiversität zu managen. Da die Einflüsse des Schnorchelns auf das Ökosystem ziemlich unbekannt sind, wurde ein Unterwasser-Schnorchel-Pfad eingerichtet, um die Schnorchler auf ein Gebiet zu konzentrieren und deren Bewusstsein für marine Lebensräume und Arten zu schärfen. Claudet et al. (2010) nutzten ein „vorher- nachher-EinflussKontrolldesign" (BACl), um zu analysieren, ob die hohe Frequentierung im Sommer einen negativen Einfluss auf das umgebende Ökosystem hat. Dazu wurden Makroalgen und die Fischartenausstattung als Indikatoren verwendet. 
Anschließend wurden permutativ multivariate Varianzanalysen (PERMANOVA) durchgeführt, um mögliche zeitliche und räumliche Veränderungen der Indikatoren zwischen dem Pfad und der Kontrollstelle im angrenzenden no-take / no-use Bereich bewerten zu können.

Einen weiteren Multi- Kriterien Ansatz verfolgen Carr \& Zwick (2005) in ihrer Fallstudie „Using GIS suitability analysis to identify potential future landuse conflicts in North Central Florida“. Wie der Titel bereits erahnen läßt, gehen die Autoren auf die Landnutzungskonflikte der drei Hauptnutzungen (Landwirtschaft, städtische Nutzung und Schutz-/ Erhaltungsfunktion) mittels einer Landnutzungskonfliktpotentialidentifikationsstrategie („Land Use Conflict Identification Strategy“- LUCIS) ein. Dafür wurde aus dem E. Odum's Compartment Model ein einfaches Landnutzungsklassifikationssystem generiert (Abbildung 9), um so die Landnutzungseignungen zu organisieren und die Landnutzungspräferenzen vergleichen zu können (Odum 1969, zitiert in Carr \& Zwick, 2005).

\begin{tabular}{|c|c|}
\hline \multicolumn{2}{|c|}{$\begin{array}{l}\text { A Strategic Approach to Future Land Use } \\
\text { Allocation Derived from the Work of Eugene P. Odum } \\
\text { in "Strategy of Ecosystem Development" }\end{array}$} \\
\hline $\begin{array}{c}\text { Odum's } \\
\text { Strategic } \\
\text { Ecosystem } \\
\text { Development } \\
\text { Land Use } \\
\text { Classes } \\
\end{array}$ & $\begin{array}{l}\text { LUCIS Future Land Use } \\
\text { Allocation Classifications }\end{array}$ \\
\hline Productive & $\begin{array}{l}\text { Agriculture - The Protection of } \\
\text { Productive Agricultural Lands }\end{array}$ \\
\hline Compromise & \multirow{2}{*}{$\begin{array}{l}\text { Conservation - The Protection of } \\
\text { Environmentally Significant Lands }\end{array}$} \\
\hline Protective & \\
\hline Urban/Industrial & $\begin{array}{l}\text { Urban-Aesthetically pleasing \& } \\
\text { functional with limits }\end{array}$ \\
\hline
\end{tabular}

Abbildung 9: Ein strategischer Ansatz zur zukünftigen Zuweisung der Landnutzung, abgeleitet von der Arbeit von Eugene P. Odum in "Strategy of Ecosystem Development" (Quelle: Carr \& Zwick, 2005).

Der Strategieprozess umfaßt sechs Schritte:

1) Die Entwicklung einer hierarchischen Reihe von Zielen und Zielsetzungen, die als Eignungskriterien fungieren.

2) Eine Bestandsaufnahme der verfügbaren Daten.

3) Die Bestimmung der Eignungen.

4) Die Kombination von Eignungen, um so Präferenzen darzustellen.

5) Die Reklassifizierung der Präferenzen in die drei Kategorien "hoch", "mittel" und "niedrig".

6) Der Vergleich der Präferenzbereiche, um die Menge und die räumliche Verteilung der möglichen Landnutzungskonflikte zu bestimmen.

Ein Ergebnis dieser Studie stellt somit die Karte der Gebiete mit und ohne potentiellen, städtischen Konflikten dar (Abbildung 10). 


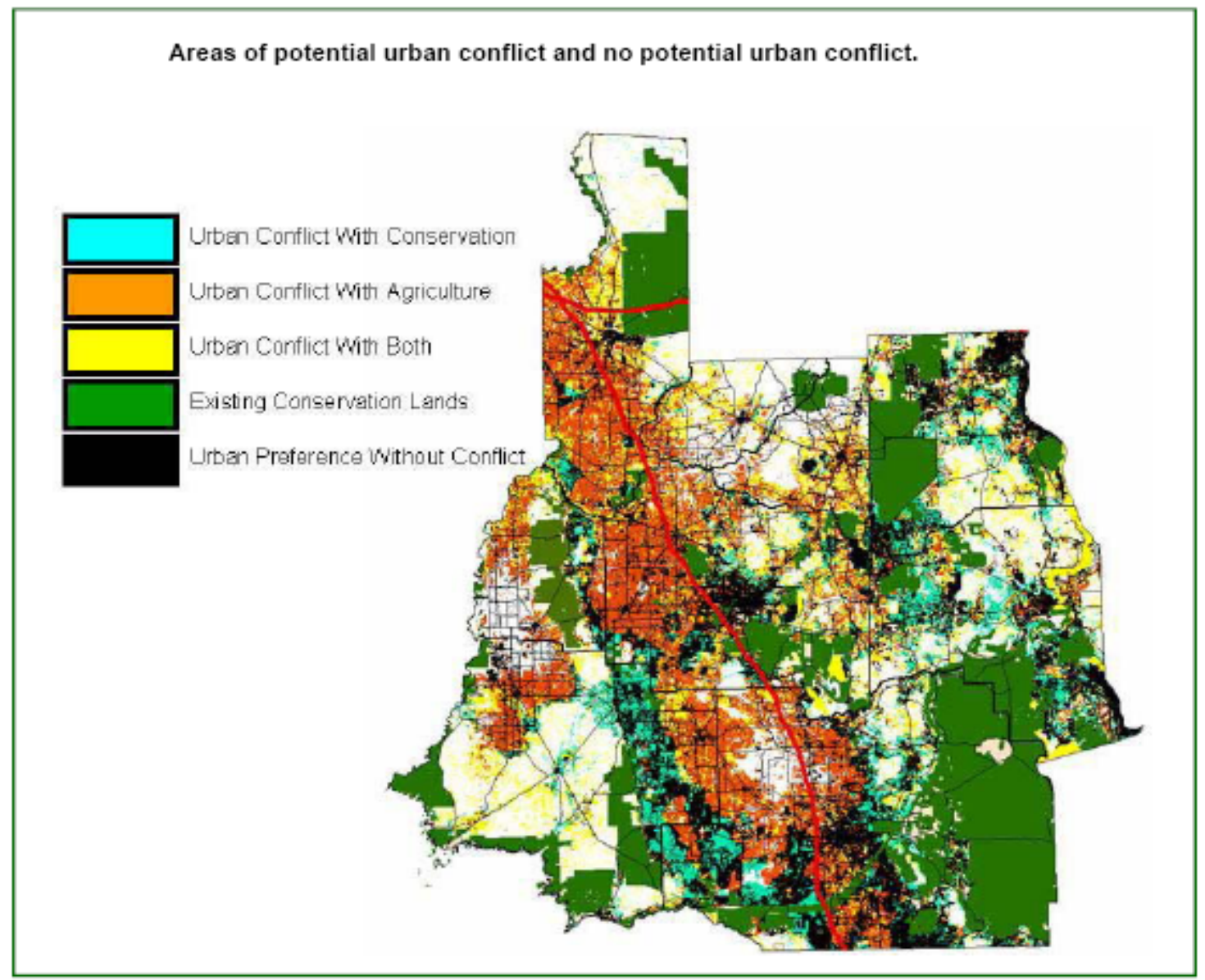

Abbildung 10: Gebiete mit und ohne potentiellen städtischen Konflikten (Quelle: Carr \& Zwick, 2005).

Ebenfalls mit mehreren Hauptkriterien beschäftigen sich Boldt \& Magun (2009) bei der „Berechnung eines integralen und artspezifischen Konfliktpotentials als Grundlage der Störungsvermeidung". Auf dieses Projekt wird detaillierter eingegangen, da dieses für das IESP Projekt von großem Interesse ist. Boldt \& Magun (2009) gehen bei ihrer Methode, die im Rahmen der Überarbeitung der Wildschutzgebiete im Kanton Bern angewendet wird, auf die Notwendigkeit von aktuellen Grundlagen für die Formulierung von Maßnahmen zur Vermeidung von Störungen der Wildtiere durch menschliche Nutzungen ein. Um diese Grundlagen zu erhalten, wurde eine Konfliktpotentialanalyse durchgeführt. So wurde eine semi- quantitative Analyse entwickelt, die in 5 Phasen (Boldt et al., 2010) auf alle Nutzungsformen eingeht und das Konfliktpotential für die wichtigsten Wildtierarten dokumentiert.

In der ersten Phase wurden die aktuellen und potentiellen Lebensräume der Wildtiere erfasst, saisonal differenziert dokumentiert und kommentiert, wobei die Auswahl der Arten für jeden Wildraum separat vorgenommen wurde. Bei den Wildtieren wurden prioritäre Arten festgelegt, die somit in die Konfliktanalyse einflossen. Dazu zählten Gämse, Steinbock, Rothirsch, Reh, Wildschwein, Feldhase, Biber, Auerhuhn, Birkhuhn, Schneehuhn, Steinadler und Wasser-/ Watvögel. Ebenfalls behandelt wurden die Arten Luchs, Wildkatze, Schneehase, Murmeltier, Haselhuhn, Bartgeier, Wanderfalke, Uhu, Kiebitz, Waldschnepfe, Weißstorch, Steinhuhn und Eisvogel. 
Das Ergebnis der ersten Phase stellten Informationen und Daten von Wildhütern und anderen lokalen Kennern, sowie aus Literatur und Datenbanken dar, die in Form von einem Bericht mit Karten und Faktenblätter (Abbildung 11) digitalisiert und visualisiert werden konnten.

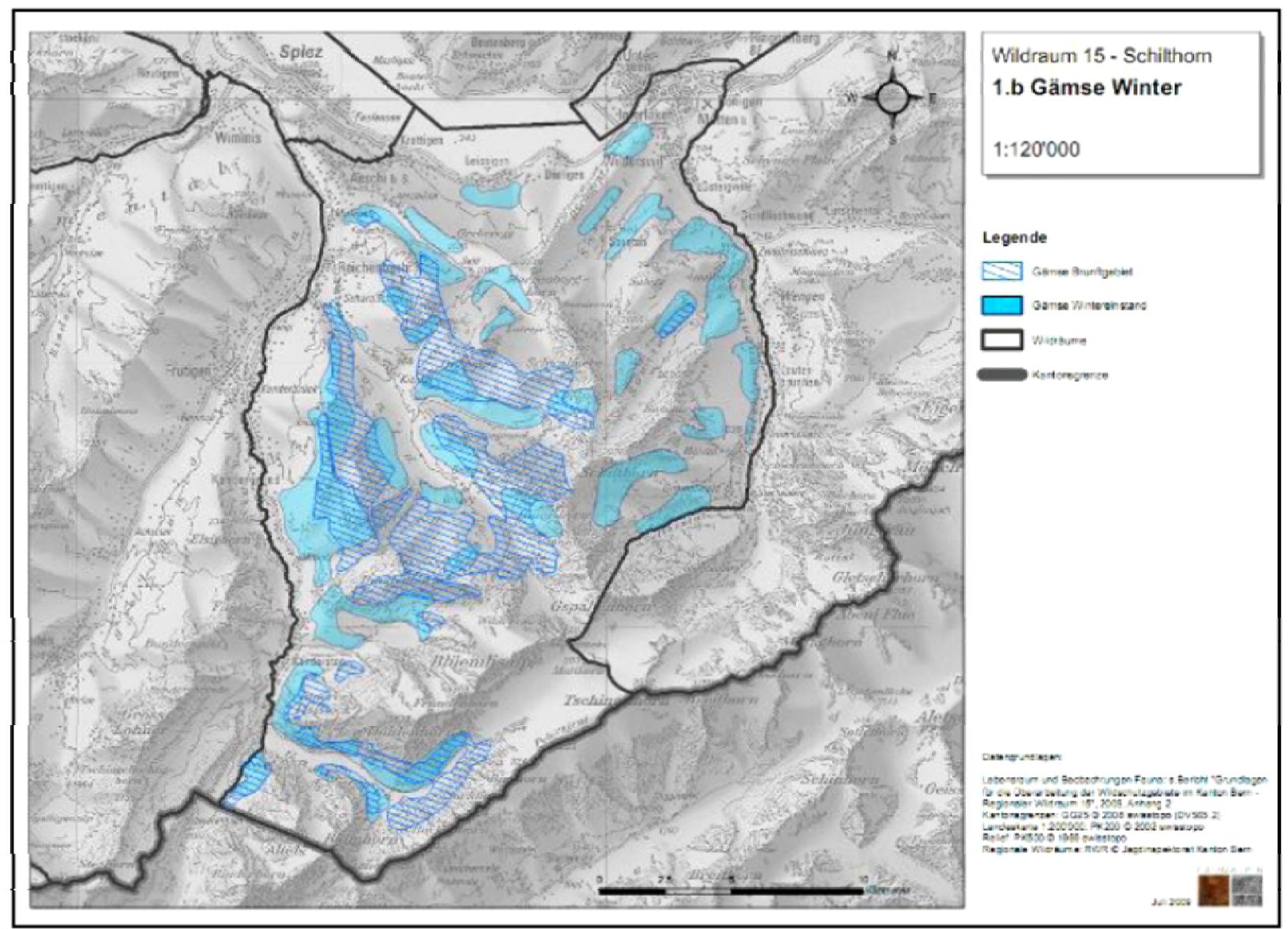

Abbildung 11: Karte über die Wintereinstände der Gämse im Wildraum 15 (Quelle: @ FaunAlpin, Bern, 2010).

In der zweiten Phase wurde die aktuelle Verbreitung und Intensität der verschiedenen Nutzungsformen semi-quantitativ erfasst, und ebenso wie die Wildtierarten saisonal differenziert dokumentiert und kommentiert. Auch hier wurde die Auswahl der Nutzungsformen für jeden Wildraum separat vorgenommen. In die Konfliktpotentialanalyse flossen die intensiv bearbeiteten Nutzungsarten Wandern, Mountainbiken, Hochtouren Klettern, Skitouren, Schneeschuhwandern, Freeriden, Wassersport und allgemeine menschliche Präsenz (inkl. Hunde) ein, weiters behandelt wurden Skifahren, Langlaufen, Winterwandern, Eisklettern, Hundeschlittenfahren, Straßenverkehr, Bahnen, Motorschlitten, Basejumping, Speedflying, Luftverkehr, Campingplätze, Siedlungen, Golfplätze, Schießplätze und Armee.

Die Datengrundlage wurde gleich wie die der Wildtiere erarbeitet, jedoch flossen hier weiters Daten aus dem touristischen Angebot, von Veranstaltern und Anbietern und aus eigenen gutachterlichen Einschätzungen ein. Das Produkt aus der zweiten Phase ist wie bei Phase Eins ein Bericht mit einer Serie von Karten und Faktenblättern. Exemplarisch stellt Abbildung 12 die Verbreitung und Intensität der Nutzungsart Wintersport im Wildraum 15 dar. 


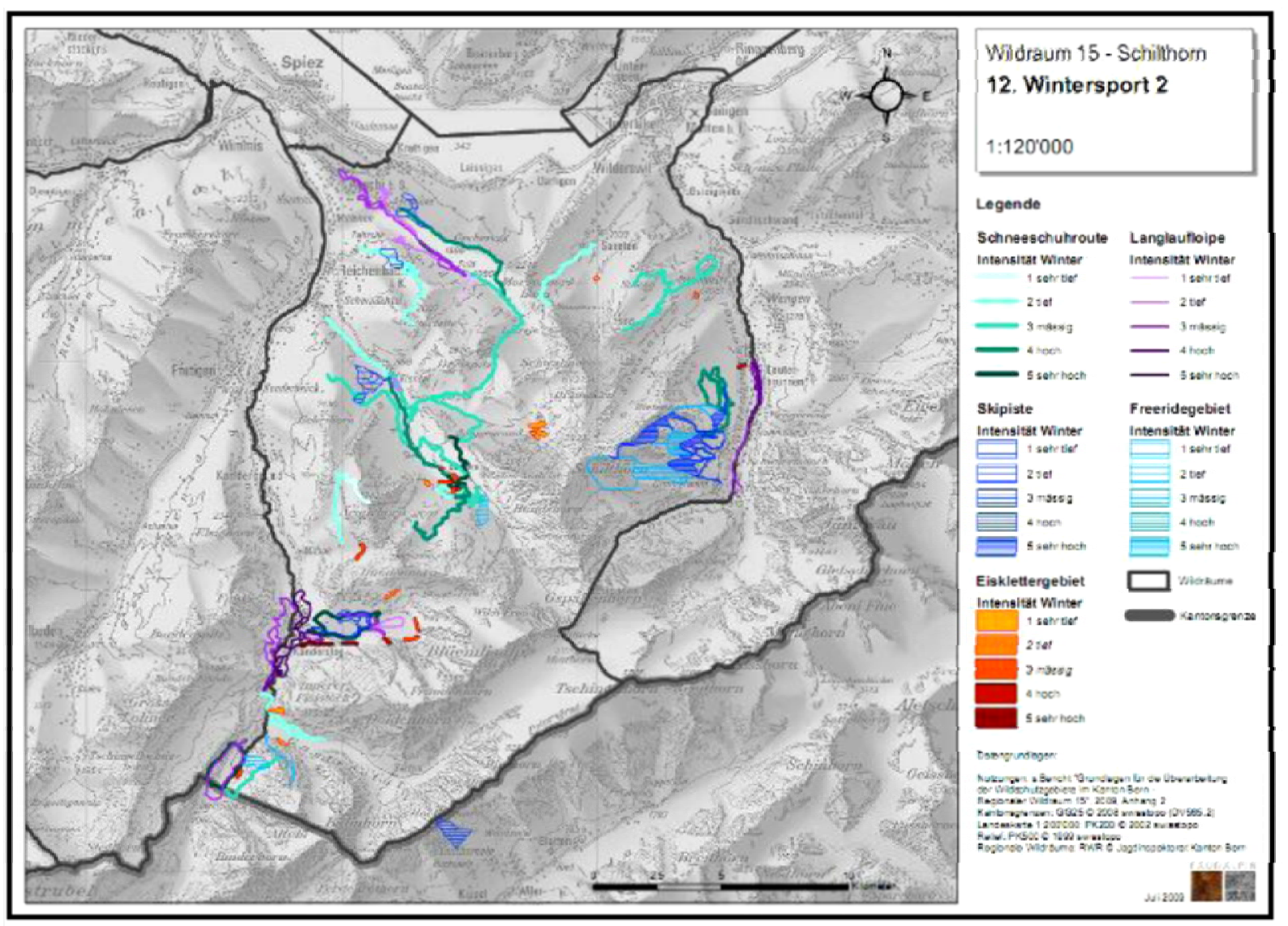

Abbildung 12: Karte über die Verbreitung und Intensität der Nutzungsart Wintersport im Wildraum 15, (Quelle: @ FaunAlpin, Bern, 2010).

Die dritte Phase umfasst die Analyse und Beurteilung mittels einer Konfliktpotentialanalyse. Hierbei wurden in einer quantitativen Analyse die Daten im GIS überlagert, wobei folgende Faktoren berücksichtigt wurden:

- Die Vorkommen der prioritären Wildtierarten

- Die Verbreitung und Intensität der wichtigsten Nutzungsformen

- Nutzungsspezifische Pufferzonen (mit zunehmender Distanz abnehmende Wirkung)

- Nutzungsspezifische Reduktionen des Potentials wie etwa des Luftverkehrs im Wald

- Art- und nutzungsspezifische Gewichtungsfaktoren (unterschiedliche Empfindlichkeit)

- Jahreszeit (Sommer/Winter)

- Räumliche Glättung und Klassifizierung in einer vierstufigen Skala

Aus dieser Überlagerung sind Konfliktkarten entstanden, die Gebiete mit hohem Konfliktpotential, sowie noch ungestörte wichtige Wildeinstände (Bsp. Gämse im Winter im Wildraum 15; Abbildung 13) zeigen. 


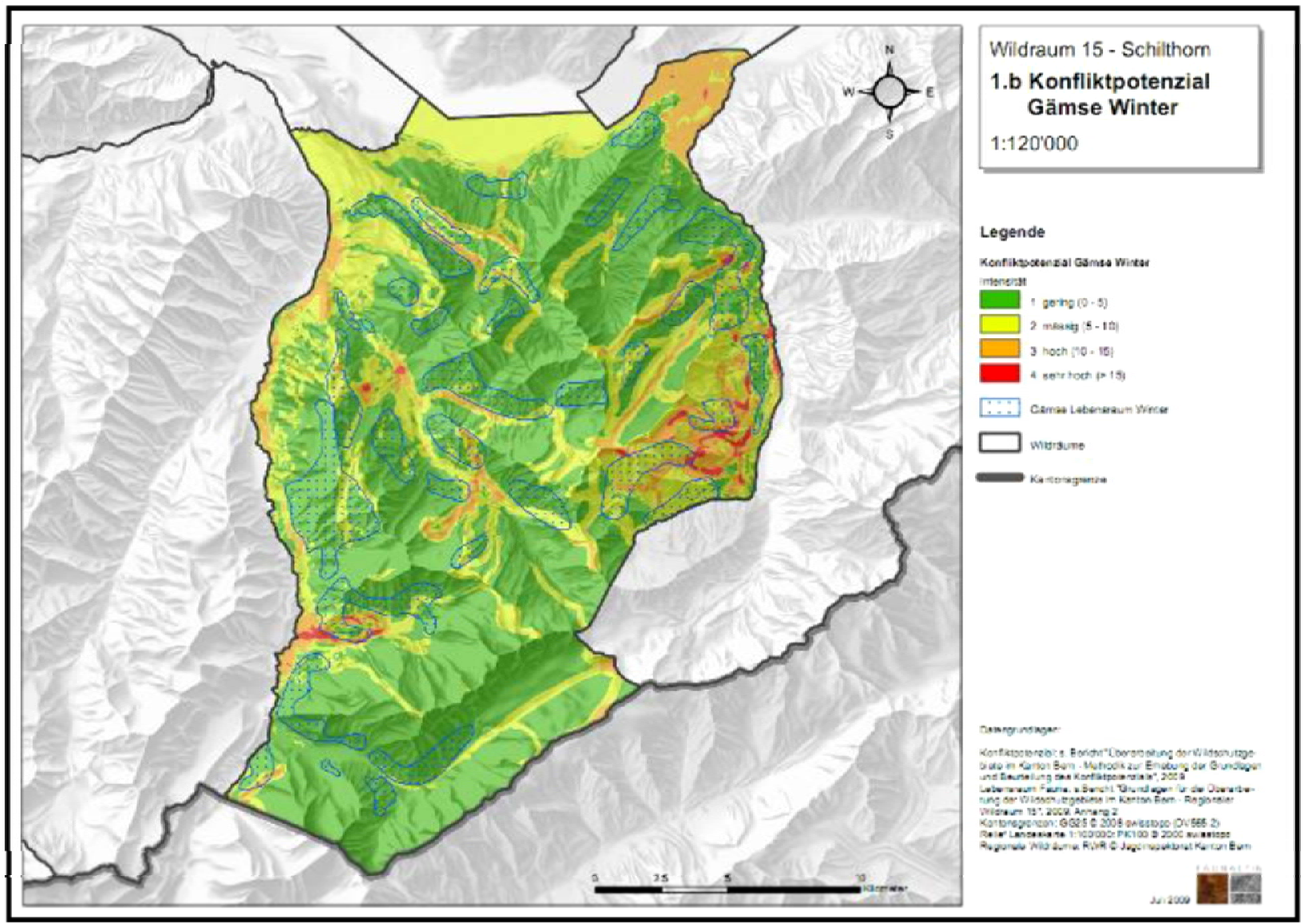

Abbildung 13: Karte über die Gämse im Winter im Wildraum 15 (Quelle: (c) FaunAlpin, Bern, 2010).

Auf Basis der Konfliktpotenzialanalyse und der Grundlagen wurden weiters Schwerpunktgebiete (SPG) innerhalb des Wildraums bezeichnet, für die gutachterliche Empfehlungen für Ziele und Maßnahmen formuliert wurden.

Anschließend wurden in Phase 4 Maßnahmen erarbeitet, die in Phase 5 in den Wildschutzgebieten im Kanton Bern umgesetzt wurden/ werden.

Die Projekte und Methoden von Boldt \& Magun (2009) und Carr \& Zwick (2005) können für das IESP Projekt interessant sein, da diese es mit Hilfe von GIS gestützten Verfahren schaffen, zum einen großräumige Gebiete auf ihre Erholungseignung hin zu bewerten und zum anderen jedoch auch intensiver auf kleinere Gebiete einzugehen. Ein weiterer positiver Aspekt dieser Projekte ist die vielfältige Erarbeitung der Datengrundlagen anhand von Literatur, Datenbanken, vorhandenem Wissen von lokalen Kennern und der Adaption von bereits bestehendem Kartenmaterial. Ebenfalls interessant für das IESP Projekt dürfte die Miteinbeziehung von mehreren Kriterien sein. 


\subsection{Grundlagen- und Datenerfassung}

\subsubsection{Experteninterviews und -konsultationen}

Die Nutzung von Expertenwissen, sowohl in regionaler als auch in fachlicher Hinsicht, spielt in dem vorliegenden Forschungsprojekt eine wichtige Rolle. Experteninterviews gehören aufgrund ihrer geringen Strukturiertheit und der offenen Befragungssituation zu den qualitativen Interviews. Anders als bei Intensivinterviews (Tiefeninterviews) steht aber nicht der Interviewpartner im Mittelpunkt der Untersuchung, sondern dessen Wissen über bestimmte Sachverhalte, die Gegenstand der jeweiligen Forschung sind. Als Experten gelten dabei beispielsweise Personen die aufgrund Ihrer Ausbildung und ausgeübten Tätigkeit über ein spezifisches Fachwissen verfügen oder die entsprechend ihrer Stellung in einem Unternehmen, einer Institution oder Organisation an Entscheidungsprozessen beteiligt sind (Mieg \& Näf, 2006). Das Interview erfolgt zumeist nach einem strukturierenden Leitfaden, der in der konkreten Interviewsituation modifiziert und ergänzt werden kann. Der Interviewer kann sich bei der Gesprächsführung auf seinen Gesprächspartner einstellen (z.B. Terminologie, Frageformulierung) und der Interviewte hat die Möglichkeit den Verlauf des Interviews mitzubestimmen. Dabei beschränken sich Experteninterviews nicht auf die reine Informationsgewinnung, sie können auch Analysen, Bewertungen oder Diskussionen beinhalten. Zu berücksichtigen ist hierbei, dass auch Experteninterviews eine Form der sozialen Interaktion darstellen. Die Ergebnisse des Experteninterviews werden somit auch durch die Beziehung zwischen Interviewer und Interviewtem sowie die Interviewsituation bestimmt.

Die im vorliegenden Projekt durchgeführten Experteninterviews bzw. -konsultationen haben eine unterschiedliche Funktion und Zielstellung. Dementsprechend wurden zumeist individuelle Interviewleitfäden und Fragenkataloge aufgestellt. Im Folgenden werden jeweils die Zielstellung und Methodik der eingesetzten Experteninterviews erläutert.

\subsubsection{Experteninterviews zu den Intensivuntersuchungsgebieten}

Bei der Bearbeitung der Intensivuntersuchungsgebiete bildeten mehrstündige Interviews mit lokalen Experten die zentrale Methode der Datenerhebung. Als Hauptinterviewpartner fungierten Managementverantwortliche auf Grundeigentümer- bzw. Bewirtschafterseite, da diese über die beste Gebietskenntnis verfügen. Darüber hinaus wurden für einige der Gebiete weitere Auskunftspersonen befragt, u. a. weitere Grundeigentümer bzw. Bewirtschafter sowie andere Personen mit Ortskenntnissen. Dabei wurden zu einem Gebiet teilweise mehrere Personen konsultiert bzw. ergänzende Interviews geführt. Der überwiegende Teil der Interviews wurde am Arbeitsort des Interviewpartners bzw. im Gebiet selbst durchgeführt, so dass im Anschluss eine Geländebegehung erfolgen konnte.

Ziel der Interviews war es, Informationen über die im Gebiet vorkommenden IndikatorWildtierarten und die ausgeübten Indikator-Freizeitaktivitäten zu gewinnen, die es ermöglichen Konfliktpotenziale zwischen Wildtiermanagement und Freizeitnutzung zu ermitteln. Hauptaufgabe der Interviews war die Darstellung der Vorkommen der einzelnen Wildtierarten und der Nutzungsintensität der ausgewählten Freizeitaktivitäten in Arbeitskarten, wobei von den Interviewpartnern jeweils eine dreistufige Graduierung vorgenommen werden sollte (vgl. Kapitel 3.3). Grundsätzlich wurden für jede Wildtierart das Hauptvorkommensgebiet und die Gebiete mit geringem Vorkommen farblich unterschieden. Die Bereiche dazwischen gelten damit als Flächen mit durchschnittlicher Vorkommensdichte und wurden ebenfalls farblich markiert. In einer weiteren Karte wurden Potentialflächen für die besprochene Art eingezeichnet. Weiterhin wurden in den Karten bekannte Barrieren und Korridore sowie Problembereiche (z. B. Wildschäden) verortet und beschrieben. Beim Freizeitbereich wurden in ähn- 
licher Weise, sowohl für die Freizeitnutzung insgesamt als auch für die einzelnen IndikatorFreizeitaktivitäten, die Areale mit intensiver Nutzung und die Areale, in denen keine oder kaum Freizeitnutzung stattfindet, dargestellt. In allen anderen Gebieten wird eine dazwischen liegende Freizeitnutzungsintensität unterstellt. Bei Bedarf wurden ebenfalls zusätzliche Informationen wie Parkplätze oder Hundeauslaufzonen erfasst. Als Kartengrundlage diente ein Ausschnitt der ÖK 50000 (ausgedruckt im Format A3), in dem die Umgrenzung des Intensivuntersuchungsgebietes und die auf diesem Kartenausschnitt befindlichen Grenzen des Biosphärenparks Wienerwald eingezeichnet waren. Der Kartenausschnitt wurde jeweils so gewählt, dass bei Bedarf auch das Umfeld des Intensivuntersuchungsgebietes einbezogen werden kann.

Eine zweite Aufgabe bestand in der Erfassung weiterer Informationen über die Wildtierarten und Freizeitaktivitäten, die für die Charakterisierung der Konfliktpotenziale von Bedeutung sind. Bei den Wildtierarten betraf dies insbesondere tages- und jahreszeitliche Unterschiede im Verhalten und die Störanfälligkeit der Wildtiere, die Rolle von Korridoren und Barrieren, Problembereiche sowie die damit verbundenen Ursache-Wirkungsgefüge. Bei den Freizeitaktivitäten wurden insbesondere tages- und jahreszeitliche Differenzierungen sowie Unterschiede in den Nutzungsmustern während der Woche und am Wochenende, Quellgebiete der Freizeitnutzer, die Störwirkung der Freizeitaktivitäten auf die Wildtiere sowie die Einhaltung von Nutzungsrestriktionen und Regelungen besprochen. Einen weiteren Aspekt bildeten Wechselwirkungen mit anderen Bereichen wie Jagd, Siedlungsentwicklung und Landwirtschaft. Ergänzend zu diesen Informationen über die untersuchten Wildtierarten und Freizeitaktivitäten wurden in den Interviews bereits ausgetragene Konflikte bzw. Konfliktpotenziale, die die Stufe eines Konfliktes erreicht haben, erfragt und in ihren grundlegenden Merkmalen (z.B. Konfliktparteien, Konfliktverlauf, Ergebnisse) beschrieben.

Der Interviewleitfaden zu den Intensivuntersuchungsgebieten besteht aus einem Wildtier-Teil und Freizeit-Teil und beinhaltet folgende Themen:

\section{Wildtier-Teil:}

- Vorkommen der Indikator-Arten im Gebiet, einschließlich jahreszeitlicher Unterschiede

- Potenziale für die betreffenden Arten

- Probleme mit den betreffenden Arten (Forstwirtschaft, Landwirtschaft, Jagd, Naturschutz, andere Bereiche), jeweils differenziert nach Jahreszeiten

- Jagdliche Aspekte (rechtliche Verhältnisse, Jagdmethoden, Jagderfolg)

\section{Freizeit-Teil}

- Intensität der Freizeitnutzung im Gebiet (ohne Differenzierung nach Freizeitaktivitäten)

- Informationen über die Erholungsuchenden und Freizeitnutzer im Gebiet (Quellgebiete, Verkehrsmittel, ...)

- Freizeitaktivitäten im Gebiet und Tendenzen ihrer Entwicklung

- Nutzungsintensitäten der Indikator-Aktivitäten im Gebiet, einschließlich jahres- und tageszeitlicher Unterschiede sowie Differenzierung zwischen Wochentag und Wochenende

- Schätzung der Nutzungshäufigkeit bei den betreffenden Freizeitaktivitäten pro Tag (in der Woche, am Wochenende)

- Informationen über die Freizeitaktivitäten bzw. Freizeitnutzer (z.B. Quellgebiete, Verkehrsmittel, Nutzungsmuster) 
- Nutzungsrestriktionen und Regelungen für die betreffenden Freizeitaktivitäten und Verhalten der Freizeitnutzer (z.B. ausgewiesene Mountainbike-Routen, nicht angeleinte Hunde, Verlassen von Wegen)

- Entwicklungspotenziale für die betreffenden Freizeitaktivitäten (Ursachen, Begründung)

- Probleme mit den betreffenden Freizeitaktivitäten aus Sicht der Wildtierarten (Störwirkungen, Verdrängungsprozesse, Maßnahmen)

- Konflikte mit den betreffenden Freizeitaktivitäten (Konfliktparteien, Konfliktprozess, Konfliktlösung)

- Konflikte der betreffen Freizeitaktivitäten mit anderen Freizeitnutzern

- Probleme der Jagd mit den betreffenden Freizeitaktivitäten

Der Ablauf der Interviews wurde so gestaltet, dass nach einer kurzen Einführung zunächst alle im Gebiet vorkommenden Wildtierarten und dann der gesamte Komplex Freizeitnutzung behandelt wurden. Die Befragungssituation war dadurch geprägt, dass in der Regel mehrere Projektmitarbeiter beteiligt waren und die beiden Teile des Interviews von unterschiedlichen Interviewern befragt wurden. Dadurch ist eine eintönige Frage-Antwort-Abfolge vermieden worden und teilweise sogar eine Diskussion entstanden. Einleitend wurden die Aufgabe des Interviews erläutert und die Arbeitskarten vorgestellt. Es erwies sich als günstiger, eine Wildtierart entsprechend des Leitfadens zunächst vollständig zu bearbeiten und danach mit der nächsten zu beginnen. Vergleiche waren allerdings jederzeit möglich. Im Freizeit-Teil wurden zunächst die Freizeitnutzung insgesamt eingeschätzt und die im Gebiet ausgeübten Freizeitaktivitäten erhoben. Erst danach wurden die sechs Indikator-Freizeitaktivitäten nacheinander behandelt. Da einerseits der Informationsbedarf in diesem Bereich sehr hoch war und andererseits die Fragen entsprechend der Spezifika der einzelnen Freizeitaktivitäten (z.B. wegebezogene Aktivitäten Mountainbiking und Joggen, Ballonfahren als Luftsport) modifiziert werden mussten, wurde im Leitfaden zwischen Leitfragen und ergänzenden Fragen unterschieden. Letztere wurden entsprechend des Interviewverlaufs einbezogen und angepasst. Die Interviews wurden protokolliert, in GIS-Arbeitskarten dokumentiert und in einer Feedback-Schleife von den ExpertInnen noch einmal kontrolliert und ggf. konkretisiert oder ergänzt.

\subsubsection{Experteninterviews zu den Indikator-Wildtierarten im Wienerwald}

Auf der Ebene des gesamten Biosphärenparks wurde die Vorgehensweise zur Erfassung der Wildtiervorkommen im Wesentlichen beibehalten. Das sichert eine Vergleichbarkeit der Informationen mit den Interviews in den Intensivuntersuchungsgebieten. Für ein Upscaling auf Bezirksebene wurden Interviews mit den Bezirksjägermeistern (Baden, Mödling, Lilienfeld, St.Pölten, Tulln, Wien-Umgebung) geführt. Die Interviews folgten dem gleichen Leitfaden wie in den Intensivuntersuchungsgebieten. Sie beinhalteten sowohl die Aufnahme der Wildtiervorkommen und weiterer raumrelevanter Informationen (z.B. Barrieren, Korridore) in Arbeitskarten als auch die Erfassung weiterer Informationen zu den Wildtierarten (z.B. Schadensgebiete, Vorkommensschwerpunkte). Einzig das Wirkungsgefüge mit dem Umland (um den besprochenen Bezirk) wurde bei diesen Interviews ausführlicher behandelt. Die Arbeitskarten wurden digitalisiert, so dass die Interviewergebnisse in die Modellierung der Vorkommenswahrscheinlichkeiten der Wildtierarten im Biosphärenpark integriert werden können.

Ergänzend hierzu wurden zu einzelnen Wildtierarten (Rotwild, Schwarzstorch, Ziesel) Fachexperten (Ornithologen, Biologen etc.) konsultiert, um Informationslücken zu schließen bzw. 
weitere Datengrundlagen zu erschließen. Diese dienten ebenfalls der Modellierung der Vorkommenswahrscheinlichkeiten der Wildtierarten. Für die Interviews wurde jeweils ein individueller Interviewleitfaden erstellt. Sie basierten zudem auf dem bereits für die Bezirksjägermeister verwendeten Kartenmaterial.

\subsubsection{Experteninterviews zu den Indikator-Freizeitaktivitäten im Wienerwald}

Im Hinblick auf die Modellierung der Nutzungswahrscheinlichkeiten der einzelnen Freizeitaktivitäten im Biosphärenpark Wienerwald wurden ergänzend zur Literaturrecherche und den Befragungsergebnissen aus dem Vorgängerprojekt (Reimoser et al., 2008) ebenfalls einige Experteninterviews durchgeführt. Diese Interviews dienten insbesondere der Beschaffung zusätzlicher Informationen über die Nutzerprofile für die einzelnen Freizeitaktivitäten (Joggen, Mountainbiking, Ballonfahren, Geocaching) und die landschaftlichen Präferenzen der Freizeitnutzer als Basis für die Definition entsprechender Parameter in der Modellierung. Die Auswahl der Interviewpartner erfolgte aufgrund ihres Wissens über die Ausübung der jeweiligen Freizeitaktivität im Wienerwald (wissenschaftliche Experten, Privatwirtschaft, Sportbzw. Tourismusorganisation). Die Interviewleitfäden zum Joggen und Mountainbiking beinhalteten Fragen zum Anteil und den sozio-demographischen Merkmalen der Freizeitnutzergruppe, zur Art und Weise wie die Freizeitaktivität ausgeübt wird, der Unterscheidung verschiedener Typen von Joggern bzw. Mountainbikern sowie zu Präferenzen und Anforderungen an bestimmte Wege und Landschaften. Außerdem wurden die Rolle ausgewiesener Routen und die Wechselbeziehungen zu anderen Freizeitaktivitäten besprochen. Bei den Freizeitaktivitäten Ballonfahren und Geocaching, für die keine Daten aus den Nutzerbefragungen im Vorgängerprojekt vorliegen, wurden zunächst grundlegende Informationen erhoben (Windrichtungen, Fahrtrouten, Fahrthöhe, Ballonvereine, Start- und Landeplätze, Geocaching-Portal).

\subsubsection{Experteninterview zur Konfliktbehandlung auf der Perchtoldsdorfer Heide}

Ansätze für eine Konfliktanalyse und erste Maßnahmen zur Konfliktbewältigung wurden bereits in den Experteninterviews zu den Intensivuntersuchungsgebieten besprochen. Dabei kristallisierte sich die Situation auf der Perchtoldsdorfer Heide als ein Good Practice Beispiel für die Konfliktaustragung und -behandlung im Wienerwald heraus.

Die Konfliktsituation auf der Perchtoldsdorfer Heide und die Maßnahmen zur Konfliktbehandlung wurden daher in einem weiteren Interview mit lokalen Experten detaillierter analysiert und diskutiert. Ziel dieses Experteninterviews war es, die Bedingungen und Akteure der Konfliktlösungsprozesse auf der Perchtoldsdorfer Heide zu beleuchten und Möglichkeiten für die Anwendung der Konfliktstrategien und -maßnahmen in anderen Gebieten bzw. im gesamten Biosphärenpark Wienerwald zu diskutieren. Der Interviewleitfaden beinhaltete die Charakterisierung der Konflikte, die jeweiligen Konfliktparteien, die Streitpunkte, die Konfliktbehandlung sowie das Konfliktergebnis und die Konfliktwirkungen. Das Interview wurde ebenfalls protokolliert. 


\subsubsection{Indikator-Freizeitaktivitäten}

\subsubsection{Auswahl der Aktivitäten}

Die Funktion von Indikatoraktivitäten innerhalb des Projekts besteht darin, Wechselwirkungen charakteristischer Freizeitaktivitäten und der Sensitivität des Naturraums anzuzeigen. Die wesentlichste Anforderung an die Auswahl der Indikatoraktivitäten bildete das Vorhandensein von räumlichem und zeitlichem Konfliktpotenzial mit den relevanten Indikatorarten. Das heißt, von Indikatoraktivitäten soll eine signifikante Störwirkung auf Indikator-Arten ausgehen, und Indikatorarten sollen erhöhte Störungssensibilität gegenüber Indikator-Aktivitäten aufweisen. Beides erfordert räumlich-zeitliche Überlagerungen in denselben Teilräumen des Biosphärenparks.

Für die Auswahl der Indikator-Freizeitaktivitäten waren vor allem folgende Auswahlkriterien maßgeblich: Die ausgewählten Aktivitäten zeigen einen erhöhten Störeinfluss gegenüber den ausgewählten Indikatorarten auf. Zudem werden die Aktivitäten häufig bis zunehmend häufig sowohl im Sommer als auch im Winter im Biosphärenpark Wienerwald ausgeführt. Die ausgewählten Aktivitäten decken linear (weggebundene), flächig (off-road) und punktuell ausgeübte Aktivitäten ab und werden vorrangig im Wald bzw. im Offenland ausgeübt. Ein weiteres Auswahlkriterium ist, dass die ausgewählten Aktivitäten auf unterschiedlichen räumlichen und (tageszeitlichen) Skalenebenen stattfinden und für die Ausübung der Aktivitäten ein unterschiedlich hoher Infrastrukturbedarf notwendig ist.

Im Einzelnen wurden folgende Auswahlkriterien definiert:

- häufig oder zunehmend häufig ausgeführte Aktivitäten

- linear (weggebunden), flächig (off-road) und punktuell ausgeübte Aktivitäten

- überwiegend im Wald und überwiegend im Offenland ausgeübte Aktivitäten

- im Sommer- und im Winterhalbjahr ausgeübte Aktivitäten

- auf unterschiedlichen räumlichen und (tages)zeitlichen Skalenebenen stattfindende Aktivitäten

- Aktivitäten mit hohem Infrastrukturbedarf

- ausreichende Datenverfügbarkeit.

Bezüglich des Infrastrukturbedarfs haben Pröbstl \& Prutsch (2009) drei Typen von Freizeitaktivitäten und Sportarten definiert, deren Ausübung sich in der Abhängigkeit von Natur und Landschaft unterscheiden. So differenzieren Pröbstl \& Prutsch (2009) zwischen:

- infrastrukturabhängigen Aktivitäten in der freien Landschaft, wie etwa der Golfsport oder der Luftsport mit speziellen Einrichtungen für den Flugbetrieb;

- auf besondere Eigenschaften der Natur und Landschaft angewiesene Aktivitäten wie etwa Downhill biking oder Klettern;

- und Aktivitäten, denen ohne spezielle Anlagen, beziehungsweise auch durch eine Mitbenutzung bestehender Infrastrukturen wie etwa von Forstwegen nachgegangen werden kann. Hierzu gehören etwa Laufen oder Langlaufen.

Durch diese unterschiedliche Abhängigkeit von Natur und Landschaft variiert auch die Wahrscheinlichkeit einer Überschneidung zwischen attraktiven Räumen für Sport und Wildtiere. 
Um auf diese Unterschiede bestmöglich einzugehen, wurden Freizeitaktivitäten aller drei von Pröbstl und Prutsch (2009) charakterisierten Typen ausgewählt.

Zudem wurde bei der Auswahl der Aktivitäten darauf geachtet, dass ausreichend Daten zu den einzelnen Aktivitäten im Biosphärenpark verfügbar sind.

Basierend auf den genannten Auswahlkriterien wurde eine erweiterte Vorauswahl von 16 potenziell geeigneten Indikator-Freizeitaktivitäten erstellt, von denen gemeinsam mit den Stakeholdern sechs Aktivitäten ausgewählt wurden:

- Mountainbiken

- Joggen

- Ballon fahren

- Geocachen

- Aktivitäten mit Hund

- Lagern und Picknicken

\section{Mountainbiken}

Diese zu allen Jahres- und Tageszeiten ausgeübte Aktivität wird sowohl linear als auch durch das Offroadfahren räumlich dispers durchgeführt. Mountainbiker nutzen sowohl Wald als auch Offenland. Das Konfliktpotenzial mit den verschiedenen Nutzergruppen des Wienerwaldes (Land- und Forstwirtschaft, Jagd, Naturschutz und auch anderen Freizeit- und Erholungssuchenden) ist hoch und auch gegenüber der Mehrheit der Indikatorarten besteht eine potenziell hohe Störintensität. Diese wird vor allem durch die Unvorhersehbarkeit der genutzten Route induziert, wodurch es zu Überraschungseffekten anstelle von Gewöhnungseffekten für die Wildtiere kommt. Mountainbiken steht als Indikatoraktivität für Schneeschuhwandern und Orientierungslauf, da sich die räumlichen Bewegungsmuster stark ähneln.

\section{Joggen}

Joggen wird zu allen Tages- und Jahreszeiten ausgeübt und findet vorrangig weggebunden statt. Landschaft und Naturnähe spielen eine wichtige Rolle bei der Wahl der Wege, wobei sowohl Wald als auch Offenland attraktiv sind. Vor allem durch Ausübung der Aktivität in der Dämmerung und in der kalten Jahreszeit kommt es zu einer Störung der Wildtiere. Joggen steht als Indikatoraktivität für Wandern, Nordic Walken und Reiten.

\section{Ballonfahren}

Ballonfahren ist nur zu bestimmten Jahres- und Tageszeiten möglich. Ballonfahrten werden ausschließlich von lizensierten Unternehmen durchgeführt. Während die Startplätze für Ballonfahrten von der Behörde frei gegeben werden müssen, sind Landungen überall erlaubt, sofern es zu keiner Beschädigung von Grund und Boden kommt. Besonders konfliktträchtig in Bezug auf Wildtiere sind Überfahrten in geringen Flughöhen sowie der Start und Ladung, da die Störeinwirkung durch Lärm und Optik am größten ist. Ballonfahren ist als Indikatoraktiviät für verschiedene Freizeitaktivitäten im Luftraum wie Drachensteigenlassen, Segelfliegerei sowie Modellflug anzusprechen. 


\section{Geocachen}

Geocaching stellt eine lineare und punktuelle und auch flächenbezogene Aktivität dar, die sowohl ontrail als auch offtrail das ganze Jahr über durchgeführt wird. Geocachen ist sowohl im Wald als auch im Offenland attraktiv, wobei Nutzer häufig in Gebiete vordringen, die bis dato vom Menschen unbeeinflusst gewesen sind. Vor allem die Suche nach dem Geocache Versteck ist durch eine lange Verweildauer in einem räumlich mehr oder weniger kleinen Bereich verbunden. Geocachen hat einen hohen Störeinfluss auf Wildtiere, da es zu keinerlei Gewöhnungseffekten kommen kann und unter Umständen sogar Lebensraum im Rahmen der Suchaktion zerstört werden kann. Geocaching steht als Indikatoraktivität für Pilze und Beeren sammeln, Blumen pflücken sowie Birdwatching und Naturfotografie.

\section{Aktivitäten mit Hund}

Unabhängig vom Wetter und der Jahreszeit müssen Hundebesitzer täglich mehrmals mit ihrem Hund ins Freie gehen. Aktivitäten mit Hund werden bevorzugt in Wohnumfeldnähe ausgeübt und weisen eine zeitliche und räumliche Regelmäßigkeit auf. Während sich die Hundeführer vorwiegend auf den Wegen bewegen, kommt es durch unangeleinte Hunde zu einem hohen Konfliktpotential mit Wildtieren, welche in Folge von Setzen von Duftmarken und auch durch Verfolgung von Wildtieren entstehen. Da Hundeführer überwiegend zu Fuß unterwegs sind, wurden für weitere Analysen nur Wanderer mit Hund berücksichtigt.

\section{Lagern und Picknicken}

Diese Freizeitaktivität konzentriert sich auf wenige Tage und Nächte im Jahr; wird dann aber von einer hohen Anzahl an Personen durchgeführt. Die Anforderungen an bevorzugt aufgesuchte Lager- und Picknickplätze sind hoch; ausgedehnte Wiesen in Waldrandnähe werden bevorzugt. Obwohl der Störradius gering ist, überlagern sich bevorzugt genutzte Picknickplätze und sämtliche Wildtierlebensräume. Diese Indikatoraktivität wurde zwar in die Überlegungen miteinbezogen, konnte jedoch nicht für die Berechnungen und Konfliktpotentialkarten herangezogen werden, da die Datengrundlage hierfür zu gering war. Lagern und Picknicken steht als Indikatoraktivität für Zelten, Rodeln, Nutzung von Spielplätzen und Lagerfeuer machen.

Eine literaturgestützte, vertiefende Betrachtung der einzelnen Indikatoraktivitäten folgt in Kapitel 3.2.2.

Eine Übersicht zur Charakterisierung der ausgewählten Indikatoraktivitäten hinsichtlich projektrelevanter Aspekte ist der nachstehenden Tabelle 4 zu entnehmen. 
Tabelle 4: Charakterisierung der Indikatoraktivitäten hinsichtlich projektrelevanter Aspekte.

\begin{tabular}{|c|c|c|c|c|c|c|c|}
\hline Indikator-Aktivitäten & $\begin{array}{l}\text { Indikatorarten } \\
\text { (mit potenziell hoher } \\
\text { Störungsintensität) }\end{array}$ & Störungsrelevanz & $\begin{array}{l}\text { indizierte } \\
\text { Konfliktaspekte }\end{array}$ & Nutzermerkmale & $\begin{array}{l}\text { Erfüllung } \\
\text { Auswahlkriterien }\end{array}$ & Schirm-Aktivität für & Anmerkung \\
\hline Mountainbiking & $\begin{array}{l}\text { - Ziesel } \\
\text { - Auerhahn } \\
\text { - Schwarzstorch (nicht im } \\
\text { Winter) } \\
\text { - Rotwild } \\
\text { - Wildschwein }\end{array}$ & 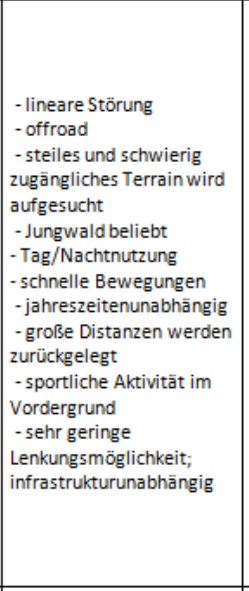 & \begin{tabular}{|l|} 
- Lebensraum- \\
zerschneidung durch Wege \\
- Uberraschungseffekt \\
\end{tabular} & 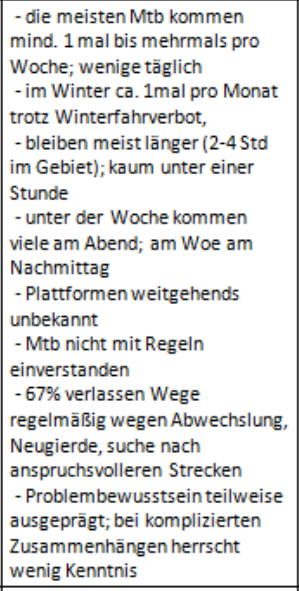 & 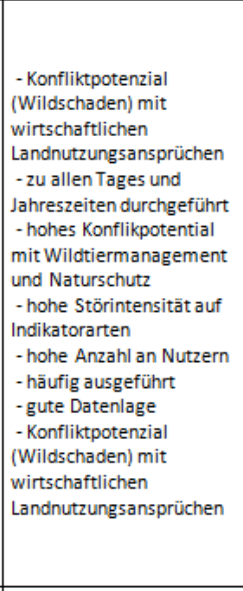 & $\begin{array}{l}\text { - Orientierungslauf } \\
\text {-Schneeschuhwandern }\end{array}$ & \\
\hline Geocaching & $\begin{array}{l}\text { - Auerhahn } \\
\text {-Schwarzstorch (nichtim } \\
\text { Winter) } \\
\text { - Rotwild } \\
\text { - Wildschwein }\end{array}$ & 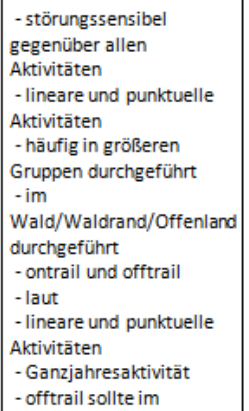 & $\begin{array}{l}\text { - es kann zu keinen } \\
\text { Gewöhnungseffekten } \\
\text { kommen, da Caches } \\
\text { vermutich immer zu } \\
\text { anderer Tageszeit und } \\
\text { anderer Routenwahl } \\
\text { aufgesuchth werden } \\
\text { - Lebensraumzerstörung } \\
\text { von Kleinsäugern und } \\
\text { wertvollen Kleinhabitaten } \\
\text { (Baumrinde, Altholz, } \\
\text { Spalten,..) durch Suche } \\
\text { und Versteck der Caches } \\
\text { - einige Caches können } \\
\text { nur in Nacht gesucht } \\
\text { werden (Reflektoren) }\end{array}$ & \begin{tabular}{|l|} 
- Zahl der Teilnehmer steigt \\
stark \\
- Teilnehmerzahl wird mit neuer \\
Mobilfunktechnologie sehr \\
rasant steigen \\
- werschiedene Plattformen für \\
WW relevant \\
- je exotischer und unüblicher \\
das Versteck desto besser - \\
- keine Lenkungsmöglichkeiten
\end{tabular} & $\begin{array}{l}\text { - hohe räumliche } \\
\text { Anforderungen } \\
\text { - zu allen Tages und } \\
\text { Jahreszeiten durchgeführt } \\
\text { - hohe Störintensitata auf } \\
\text { Indikatorarten } \\
\text { - hohes Konflikpotential } \\
\text { mit Wildtiermanagement } \\
\text { und Naturschutz }\end{array}$ & $\begin{array}{l}\text { - Pilze und Beeren sammeln } \\
\text { - Blumen pflücken } \\
\text { - Birdwatching (ev.) } \\
\text { - Naturphotografie (ev.) }\end{array}$ & $\begin{array}{l}\text { Birdwatching und } \\
\text { Naturphotografie sind } \\
\text { von der Bewegung und } \\
\text { dem Verhalten gleich; } \\
\text { allerdings ist } \\
\text { anzunehmen, dass diese } \\
\text { beiden Nutzergruppen } \\
\text { ein öhheres } \\
\text { Problembewusstsein } \\
\text { gegenüber } \\
\text { Wildtierstörungen } \\
\text { haben als Geocacher }\end{array}$ \\
\hline
\end{tabular}

IESP - Towards Integrated Ecological Spatial Planning for the Wienerwald Biosphere Reserve 


\begin{tabular}{|c|c|c|c|c|c|c|c|}
\hline Indikator-Aktivitäten & \begin{tabular}{|l} 
Indikatorarten \\
(mit potenziell hoher \\
Störungsintensität)
\end{tabular} & Störungsrelevanz & $\begin{array}{l}\text { indizierte } \\
\text { Konfliktaspekte }\end{array}$ & Nutzermerkmale & $\begin{array}{l}\text { Erfüllung } \\
\text { Auswahlkriterien }\end{array}$ & Schirm-Aktivität für & Anmerkung \\
\hline & & $\begin{array}{l}\text { Allgemeinen gut begehbar } \\
\text { sein durchgeführt } \\
\text { - Zerstörung von Höhlen, } \\
\text { Bäumen usw. möglich } \\
\text { - keine } \\
\text { Lenkungsmöglichkeiten } \\
\text { derzeit } \\
\text { - längeres Verharren auf } \\
\text { selber Stelle }\end{array}$ & $\begin{array}{l}\text { - Teilnehmer lernen neue } \\
\text { Platze abseits der Wege } \\
\text { kennen und verbreiten } \\
\text { diese im Web wodurch } \\
\text { Frequenz erhöht wird }\end{array}$ & & & & \\
\hline Aktivität mit Hund & $\begin{array}{l}\text {-Ziesel } \\
\text { - Auerhahn } \\
\text {-Schwarzstorch (nicht im } \\
\text { Winter) } \\
\text { - Rotwild } \\
\text { - Wildschwein (nur } \\
\text { Jagdhunde) }\end{array}$ & $\begin{array}{l}\text { - bei allen Nutzungen wird } \\
\text { Hund häufig } \\
\text { mitgenommen } \\
\text { - oft nicht an der Leine } \\
\text { - Ganzjahresaktivität } \\
\text { - zu allen Jahreszeiten }\end{array}$ & $\begin{array}{l}\text { - Störradius entlang der } \\
\text { Wege erhöht sich } \\
\text { - Aufschnüffeln// } \\
\text { Verfolgung von Wildtieren }\end{array}$ & $\begin{array}{l}\text { - ca. } 8 \% \text { der Jogger haben Hund } \\
\text { mit-nur wenige davon sind } \\
\text { angeleint } \\
\text { - rund } 17 \% \text { der Wanderer haben } \\
\text { Hund mit; Halfte davon } \\
\text { unangeleint } \\
\text { - tw auch Reiter und Mtb mit } \\
\text { Hund unterwegs } \\
\text { - geringes Problembewusstsein }\end{array}$ & $\begin{array}{l}\text { - zu allen Tages und } \\
\text { Jahreszeiten durchgeführt } \\
\text { - hohes Konflikpotential } \\
\text { mit Wildtiermanagement } \\
\text { und Naturschutz } \\
\text { - hohe Störintensitāt auf } \\
\text { Indikatorarten } \\
\text { - hohe Anzahl an Nutzern } \\
\text { - häufig ausgeführt } \\
\text { - mäßßsig bis gute Datenlage }\end{array}$ & $\begin{array}{l}\text { - Joggen mit Hund } \\
\text { - Mountainbiken mit Hund } \\
\text { - Reiten mit Hund } \\
\text { - Geocaching mit Hund } \\
\text { - Lagern, Picknicken mit } \\
\text { Hund }\end{array}$ & \\
\hline Lagern, Picknicken & $\begin{array}{l}\text {-Ziesel } \\
\text {-Auerhahn } \\
\text {-Schwarzstorch }\end{array}$ & $\begin{array}{l}\text { - konzentriert auf wenige } \\
\text { Tage im Jahr } \\
\text { - eingeschränkte Lenkung } \\
\text { möglich } \\
\text { - halten sich am liebsten } \\
\text { am Waldrand auf } \\
\text { - eher Sommeraktivitāta } \\
\text { - Tag und Nacht } \\
\text { - laut } \\
\text { - Müllverschmutzung, } \\
\text { Eutropisierung, }\end{array}$ & 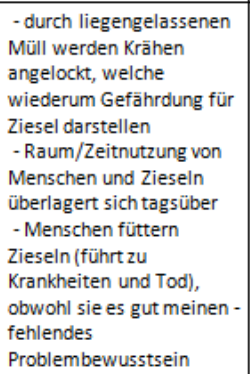 & $\begin{array}{l}\text { - kein Nutzerprofil vorhanden } \\
\text {-keine Nutzerplattformen } \\
\text { vorhanden } \\
\text { - kaum Lenkungsmöglichkeiten }\end{array}$ & $\begin{array}{l}\text { - zu allen Tageszeiten } \\
\text { durchgeführt } \\
\text { - hohe Strörintensitāta auf } \\
\text { Indikatotoraten } \\
\text { - hohe Anzahl an Nutzern } \\
\text { - hāufig ausgeführt } \\
\text { - hohe rāumliche } \\
\text { Anforderungen }\end{array}$ & $\begin{array}{l}\text { - Lagerfeuer } \\
\text { - - - Spachensteigen } \\
\text { - Bob fatatz } \\
\text { - Drachensteigen } \\
\text { - - - pielplatz } \\
\text { - -elten } \\
\text { - Zelffeste }\end{array}$ & \\
\hline
\end{tabular}




\subsubsection{Angewandte Methoden zur Datenerhebung}

Zu den ausgewählten Freizeitaktivitäten wurden Literaturrecherchen durchgeführt. Ziel der Literaturrecherche war, die Erstellung eines soziodemografischen Nutzerprofils sowie die Darstellung der räumlichen und zeitlichen Anforderungen für jede Indikatoraktivität. Des Weiteren sollte anhand der Literatur aufgearbeitet werden, welche Nutzermotive den Erholungssuchenden der jeweiligen Freizeitaktivität zu Grunde liegen und welches Problembewusstsein die Nutzer gegenüber möglichen Störeinflüssen in Bezug auf die Wildtiere haben.

Weiteres wurden die Ergebnisse der Nutzerbefragung, welche im Rahmen des Vorgängerprojekts „Integriertes nachhaltiges Wildtiermanagement im Biosphärenpark Wienerwald“ (Reimoser et al., 2008) durchgeführt worden ist, herangezogen, um die Nutzerprofile der Jogger und Mountainbiker sowie Personen, die mit Hunden unterwegs sind, mit biosphärenparkbezogenen Angaben zu ergänzen bzw. konkretisieren zu können. Für die Indikatoraktivitäten Geocachen, Picknicken und Lagern sowie Ballonfahren stehen keine Daten aus der durchgeführten Nutzerbefragung zur Verfügung.

Zur Ergänzung der Literaturrecherchen wurden mehrere Interviews mit ausgewählten Experten durchgeführt. Die Experten wurden vor allem hinsichtlich ihres Wissens in Bezug auf die jeweilige Indikatoraktivität ausgewählt und stellten sowohl wissenschaftliche Experten, Behördenvertreter als auch Fachkundige aus der Privatwirtschaft dar. Die Experteninterviews wurden mit Hilfe strukturierter, nicht standardisierter Interviewleitfäden durchgeführt. Die Interviews waren vor allem dahingehend ausgerichtet, noch fehlende Informationen aus den Literaturrecherchen zu ergänzen und eine gute Datenbasis für die weitere Projektbearbeitung herzustellen.

\subsubsection{Modellierung der Nutzungswahrscheinlichkeiten der Indikatoraktivitäten mit- tels GIS}

Grundlage für die biosphärenparkweite Konfliktpotenzialanalyse bildet die Abschätzung der räumlichen Verteilung von Nutzungsintensitäten der Freizeit-Indikatoraktivitäten für den gesamten Biosphärenpark. Eine Vielzahl von Eignungskriterien beschreibt dabei die Zielsetzung, nämlich die Wahrscheinlichkeit der Nutzerwahrscheinlichkeit der Erholungssuchenden im Biosphärenpark Wienerwald. Für jede Erholungsaktivität wird eine eigene Analyse durchgeführt.

Die Eignungskriterien der einzelnen Indikatoraktivitäten setzen sich aus Parametern der Quell- und Zielgebiete der einzelnen Indikatoraktivitäten zusammen. Zu den Quellgebietsparametern zählen die demografische Verteilung, der Aktionsradius der einzelnen Indikatoraktivitäten sowie die Erschließung des Zielgebiets vom Quellgebiet ausgehend, während zu den Zielgebietsparametern sozio-psychografische Kriterien und Landschaftskriterien zählen. Die Parameter wurden auf Basis von Literaturrecherchen, den Ergebnissen aus Befragungen (vgl. Reimoser et al., 2008) sowie mit Hilfe von Experteninterviews bestimmt.

Die definierten Parameter wurden in weiterer Folge im GIS aufbereitet, nach deren Bedeutung für die Nutzungswahrscheinlichkeit gewichtet und miteinander überlagert. Das Ergebnis der Überlagerung ist eine abgestufte Darstellung der Nutzungswahrscheinlichkeit für jede betrachtete Indikatoraktivität.

Um durch die Bildung von scharfen Grenzen in Übergangsbereichen Fehlinterpretationen auszuschließen, wurde bei der graphischen Darstellung fließenden Grenzen gegenüber einer starren Klasseneinteilung der Vorzug gegeben. 
Die resultierenden Ergebniskarten wurden über die Referenzdaten der Intensivuntersuchungsergebnisse überprüft.

Als Ergebnis dieses Arbeitsschrittes liegen Kartensätze vor, wobei die einzelnen Karten die Darstellung der Nutzungswahrscheinlichkeit für jede Indikator-Freizeitaktivität für den Biosphärenpark zeigen. Je nach Indikatoraktivität wird entweder die Nutzungswahrscheinlichkeit für das Wochenende oder für „unter der Woche“ dargestellt. Die Aktivitäten Mountainbiken, Joggen und Aktivität mit Hund zeigen die Nutzung unter der Woche, während Geocachen für alle Wochentage und Ballonfahren für das Wochenende modelliert wurden. Die Darstellung der Nutzungswahrscheinlichkeiten von Mountainbiken, Joggen und Aktivitäten mit Hund für das Wochenende ist mit den im Projekt zur Verfügung stehenden Daten nicht möglich, da unter anderem Daten über Besucher- und Verkehrsströme für den Biosphärenpark und darüber hinausgehend notwendig wären.

\subsubsection{GIS Einsatz in der Modellierung der Erholungs- und Freizeitwirtschaft}

Seit ca. 20 Jahren werden GIS basierte Analysen zur Bewertung der Eignung unterschiedlicher Standorte eingesetzt. Mit Hilfe von GIS ist es möglich, Informationen räumlich verortet darzustellen und zu analysieren. GIS stellt somit ein leistungsfähiges Werkzeug von raumbezogenen Daten dar, die in einem räumlichen, thematischen oder zeitlichen Bezug erfasst sind.

Vor allem im Rahmen der Bearbeitung regionalanalytischer Fragestellungen (u.a. Darstellung der Erholungsnutzung) spielt GIS eine große Rolle. Da die Erholungsnutzung eines Raums eng mit den räumlichen Gegebenheiten verzahnt ist (Anreisedauer und -zeit, Attraktivität eines Raums, Geomorphologie...) (Bhat G. \& Bergstom J., 1997), stellt die Nutzung von GIS ein anerkanntes und gut etabliertes Werkzeug dar, um die Bearbeitung von Managementfragen in Bezug auf die Erholungsnutzung in Schutzgebieten zu unterstützen (Longley et al., 2001; Gimblett, 2002). Am häufigsten werden in der Analyse von Erholungsnutzung bzw. -nachfrage folgende GIS-basierte Methoden angewendet: Abfrageanalyse (Query Analysis), Überlagerungsanalysen (Overlay Analysis), Routenanalysen (Routing Analysis), Dichteanalysen (density analysis) sowie Modellierung und Simulation.

Diese verschiedenen Analysemethoden werden in der Regel kombiniert angewendet und beinhalten sowohl die Überlagerung räumlicher Daten als auch die Verknüpfung von räumlichen Daten über Datenbanken. Mit Hilfe dieser angeführten Methoden können unter anderem Eignungsanalysen in Bezug auf die Erholungsnutzung durchgeführt werden, in welchen die Eignung eines Raums für die Erholungsnutzung abgebildet wird. Durch die Analyse komplexer Wirkungsgefüge und Interaktionen zwischen den verschiedenen einflussnehmenden Parametern wird somit Entscheidungsträgern und Planern ein hilfreiches Tool im Planungsprozess gegeben (Steiner et al, 2000).

Taczanowska et al. (2010) haben sämtliche GIS basierte Methoden zusammengetragen, die für die räumliche Analyse von Erholungssuchenden eingesetzt werden (Tabelle 5): 
Tabelle 5: Übersicht über GIS basierte Methoden zur Analyse von Besucherströmen (Quelle: verändert nach Taczanowska et al., 2010).

\begin{tabular}{|c|c|c|}
\hline \multicolumn{2}{|l|}{ Analyse Methode } & \multirow{2}{*}{$\begin{array}{l}\text { Anwendungsbeispiel } \\
\text { Darstellung der räumlichen Verteilung einer } \\
\text { bestimmten Nutzergruppe (Erholungsnutzende } \\
\text { in der Früh, Mountainbiker,...) }\end{array}$} \\
\hline Auswahl & thematische Auswahl & \\
\hline & räumliche Auswahl & $\begin{array}{l}\text { Identifikation von Konfliktzonen durch Überla- } \\
\text { gerung von intensiv genutzten Gebieten und } \\
\text { ökologisch sensiblen Gebieten }\end{array}$ \\
\hline \multirow[t]{3}{*}{ Aggregation } & \multirow[t]{2}{*}{ Summenbildung } & $\begin{array}{l}\text { Berechnung der Gesamtnutzerzahl pro Ab- } \\
\text { schnitt }\end{array}$ \\
\hline & & Berechnung individueller Routenlängen \\
\hline & Überlagerung & $\begin{array}{l}\text { Identifikation von Konfliktzonen durch Überla- } \\
\text { gerung von intensiv genutzten Gebieten und } \\
\text { ökologisch sensiblen Gebieten }\end{array}$ \\
\hline \multirow[t]{4}{*}{$\begin{array}{l}\text { Distanz basierte } \\
\text { Analyse }\end{array}$} & \multirow[t]{2}{*}{ Netzwerkanalyse } & $\begin{array}{l}\text { Berechnung eines Einzugsgebietes eines Er- } \\
\text { holungsgebietes }\end{array}$ \\
\hline & & $\begin{array}{l}\text { Distanzberechnungen zwischen Quell- und } \\
\text { Zielgebiet }\end{array}$ \\
\hline & Distanzanalyse & $\begin{array}{l}\text { Berechnung der Distanz zu einem spezifischen } \\
\text { Objekt (Wegstück, Fluss, Infrastruktur, etc.) }\end{array}$ \\
\hline & Pufferzonen & $\begin{array}{l}\text { Positionieren eines Puffers um ein spezifisches } \\
\text { Objekt (Weg, Fluss, etc.) }\end{array}$ \\
\hline Dichteanalyse & $\begin{array}{l}\text { Dichteanalyse } \quad \text { Ker- } \\
\text { nel/einfache Analyse) }\end{array}$ & $\begin{array}{l}\text { Dichteberechnung von GPS Wegpunkten, } \\
\text { Nächtigungen, etc. }\end{array}$ \\
\hline $\begin{array}{l}\text { Räumlich- } \\
\text { zeitliche Analyse }\end{array}$ & Streckennachverfolgung & $\begin{array}{l}\text { Aufzeigen der räumlich-zeitlichen Bewe- } \\
\text { gungsmuster von Erholungssuchenden }\end{array}$ \\
\hline
\end{tabular}

\subsubsection{Vorhandene Daten}

Für die Modellierung der Nutzungswahrscheinlichkeit standen GIS Daten des Landes Niederösterreich, Daten der Bundesforste, Daten des Biosphärenparks Wienerwald sowie Datensätze, die im Zuge des Projekts „Detailplanung Biosphärenpark Wienerwald“ (Kirchmeir et al., 2004) entstanden sind, zur Verfügung.

Die verwendeten GIS Daten wurden zuerst in dasselbe Referenzsystem (MGI 34) transformiert und bereinigt (einzelne Layer wurden zusammengeführt, doppelte Einträge gelöscht, Attribute zusammengefasst). Weiteres wurden die Datensätze teilweise adaptiert und ver- 
vollständigt sowie mit demografischen Informationen der Statistik Austria „Ein Blick auf die Gemeinde" (www.statistik.at, 2010) verknüpft.

Die Herkunft der sonstigen Daten und Informationen, die sich auf die einzelnen Indikatoraktivitäten beziehen und in die Analyse mit einbezogen wurden ist in der Tabelle 6 ersichtlich.

Tabelle 6: Datengrundlagen

\begin{tabular}{|c|c|c|c|}
\hline Inhalt & Format I & Format II & Herkunft \\
\hline $\begin{array}{l}\text { Grenzen } \\
\text { BPWW Aussengrenze } \\
\text { Gemeindegrenzen NÖ } \\
\text { Bezirksgrenzen Wien }\end{array}$ & $\begin{array}{l}\text { Vektor } \\
\text { Vektor } \\
\text { Vektor }\end{array}$ & $\begin{array}{l}\text { Polygon } \\
\text { Polygon } \\
\text { Polygon }\end{array}$ & $\begin{array}{l}\text { BPWW } \\
\text { Land Niederösterreich } \\
\text { Stadt Wien }\end{array}$ \\
\hline $\begin{array}{l}\text { Flächennutzung, Widmung } \\
\text { Baulandumhüllende }\end{array}$ & Vektor & Polygon & Land Niederösterreich \\
\hline $\begin{array}{l}\text { Wegenetz } \\
\text { Strassennetz } \\
\text { Forststraßen }\end{array}$ & $\begin{array}{l}\text { Vektor } \\
\text { Vektor }\end{array}$ & $\begin{array}{l}\text { Linie } \\
\text { Linie }\end{array}$ & $\begin{array}{l}\text { E.C.O. } \\
\text { E.C.O. }\end{array}$ \\
\hline $\begin{array}{l}\text { Gewässer } \\
\text { Flüsse } \\
\text { stehende Gewässer }\end{array}$ & $\begin{array}{l}\text { Vektor } \\
\text { Vektor }\end{array}$ & $\begin{array}{l}\text { Linie } \\
\text { Polygon }\end{array}$ & $\begin{array}{l}\text { Land Niederösterreich } \\
\text { Land Niederösterreich }\end{array}$ \\
\hline $\begin{array}{l}\text { Vegetation } \\
\text { Waldbedeckung }\end{array}$ & Vektor & Polygon & E.C.O. \\
\hline $\begin{array}{l}\text { Aktivitäten } \\
\text { Lage Geocaches } \\
\text { Mountainbikerouten } \\
\text { Wanderwege } \\
\text { Joggingstrecken }\end{array}$ & $\begin{array}{l}\text { Vektor } \\
\text { Vektor } \\
\text { Vektor } \\
\text { Vektor }\end{array}$ & $\begin{array}{l}\text { Punkt } \\
\text { Linie } \\
\text { Linie } \\
\text { Linie } \\
\end{array}$ & $\begin{array}{l}\text { http://aj-gps.net/ } \\
\text { www.mtbwienerwald.at } \\
\text { E.C.O. } \\
\text { www.runmap.net }\end{array}$ \\
\hline $\begin{array}{l}\text { Gelände } \\
\text { Digitales Geländehöhenmodell }\end{array}$ & Raster & $25 \times 25$ & $\begin{array}{l}\text { Bundesamt für Eich- und Ver- } \\
\text { messungswesen (BEV) }\end{array}$ \\
\hline $\begin{array}{l}\text { Demographische Daten } \\
\text { Altersverteilung } \\
\text { Bildungsgrad } \\
\text { Geschlecht }\end{array}$ & & & $\begin{array}{l}\text { Statistik Austria } \\
\text { Statistik Austria } \\
\text { Statistik Austria }\end{array}$ \\
\hline $\begin{array}{l}\text { Sonstige Daten } \\
\text { Registrierte Hunde } \\
\text { Niederöstereich } \\
\text { Registrierte Hunde Wien } \\
\text { Windverteilung Wienerwald }\end{array}$ & & & $\begin{array}{l}\text { Heimtierdatenbank } \\
\text { Stadt Wien, Magistratsabteilung } \\
6 \\
\text { www.zamg.at }\end{array}$ \\
\hline
\end{tabular}




\subsubsection{Methode}

Jeder Quell- und Zielgebietsparameter für die Indikatoraktivitäten wurde für die weitere Bearbeitung in einer Rasterkarte dargestellt. Eine Rasterkarte ist eine Matrix aus einzelnen Rasterelementen (Pixel), welchen ein Wert zugeordnet werden kann. Jeder Pixel entspricht dabei einer zu definierenden Fläche in der Natur, wobei in der vorliegenden Studie ein Pixel einer Fläche von 25x25 m entspricht. Jene Flächen, welche für einen Quell- oder Zielgebietsparameter auf eine hohe Nutzungswahrscheinlichkeit schließen lassen (z. B.: Nähe zu bebauter Fläche mit vielen Einwohnern, hohe Dichte an Mountainbikerouten, hohe Dichte an Geocaches,...) bekommen einen größeren Wert zugewiesen als jene auf denen eine geringere Nutzungswahrscheinlichkeit zu erwarten ist.

Um die einzelnen Parameter (z. B. Hangneigung und soziodemographische Daten, wie Bevölkerungsdichte, Alter, Bildung, etc.) miteinander vergleichbar zu machen, wurde den einzelnen Bildpunkten jedes Layers eine numerische Indexskala von 1-100 zugewiesen. Je höher der zugewiesene Wert für einen Bildpunkt ist, desto höher ist die Eignung für die jeweilige Aktivität an der betreffenden Stelle.

Das folgende Beispiel soll die Vorgehensweise verdeutlichen:

Je näher sich ein Punkt an der bebauten Fläche (die rote Fläche in Abbildung 14 stellt jene bebauten Gebiete dar, in denen zwischen 3001 und 4000 männliche Bewohner im Alter zwischen 15 und 30 Jahren leben) befindet, desto größer ist die Wahrscheinlichkeit (dunkle Flächen) Mountainbiker an diesem Punkt anzutreffen. Je weiter ein Punkt von der bebauten Fläche entfernt ist, desto unwahrscheinlicher (hellere Bereiche) ist die Nutzung durch Mountainbiker aus dem Quellgebiet. Nach überschreiten des generellen Aktionsradius von Mountainbikern ist die Aufenthaltswahrscheinlichkeit dementsprechend sehr gering (gelbe Bereiche).

Die so entstandenen Einzelkarten für alle Kriterien wurden im Anschluss daran nach deren Relevanz für die betrachtete Aktivität literaturgestützt gewichtet (z.B. Gemeinde mit einer niedrigen Einwohnerzahl erhalten einen geringe Gewichtung, Gemeinden mit hohen Einwohnerzahl einen Höhere) und nach der Methode der direkten Gewichtung aufsummiert. 


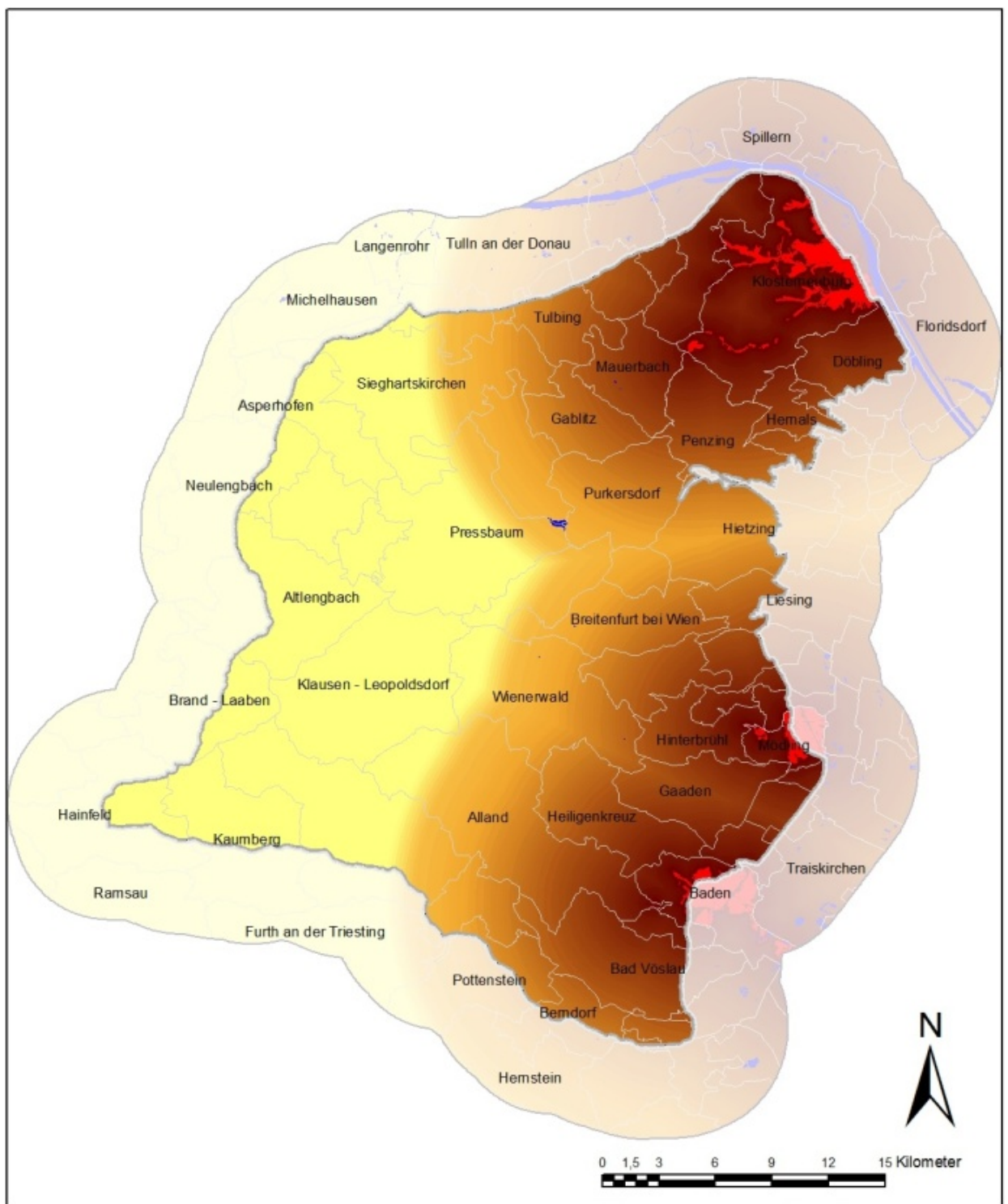

Abbildung 14: Beispiel für Distanzberechnung (Plangrundlage: BPWW, Land Niederösterreich, Statistik Austria)

Abbildung 15 verdeutlicht die Gewichtung und Aufsummierung der einzelnen Kriterien am Beispiel der Nutzungswahrscheinlichkeit für das Mountainbiken: 


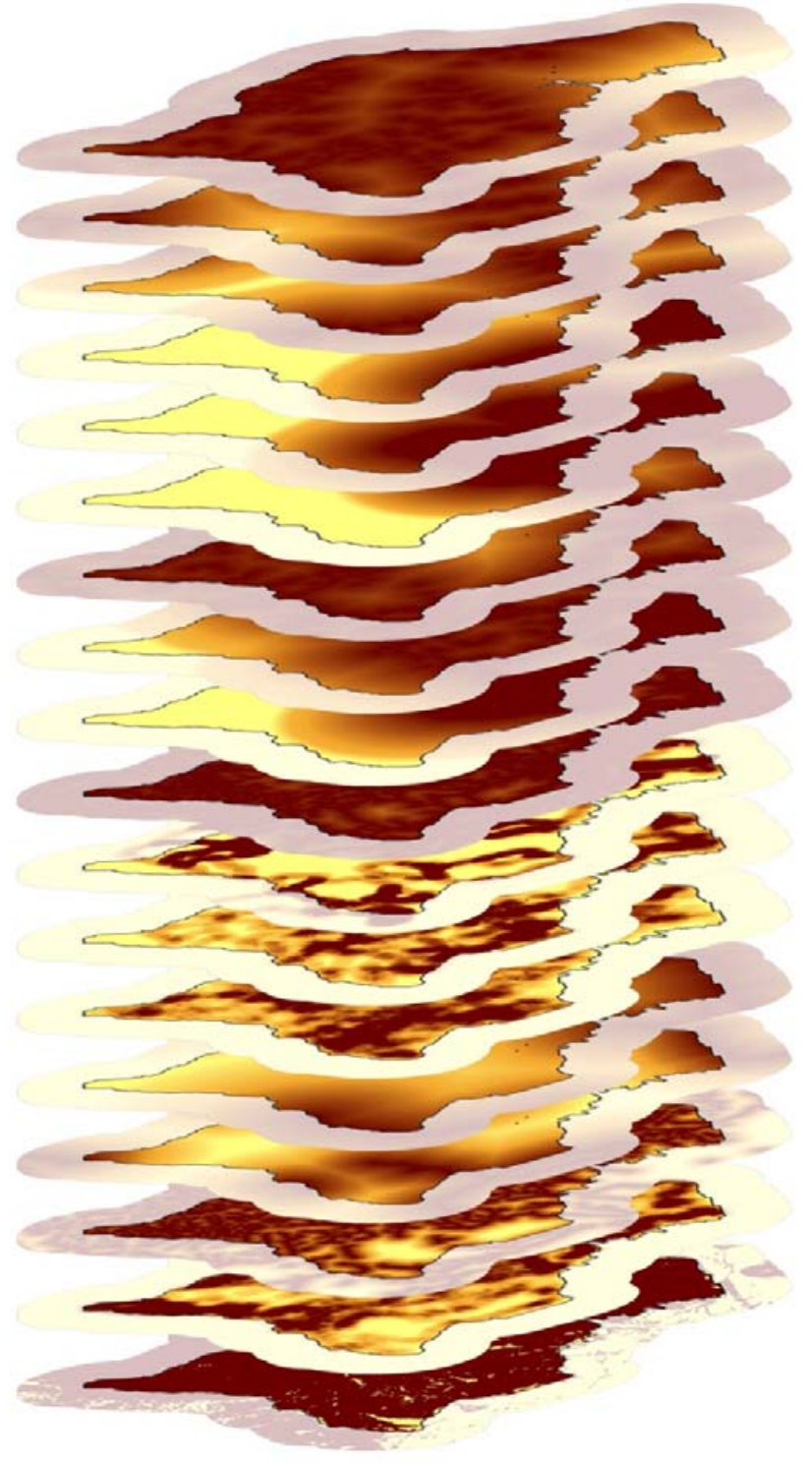

Distanz zu Bauland nach Alter und Geschlecht Klasse $1 * 0,008$

$+\quad$ Distanz zu Bauland nach Alter und Geschlecht Klasse $2 * 0,017$

$+\quad$ Distanz zu Bauland nach Alter und Geschlecht Klasse $3 * 0,025$

Distanz zu Bauland nach Alter und $+\quad$ Geschlecht Klasse $4 * 0,033$

Distanz zu Bauland nach Alter und

$+\quad$ Geschlecht Klasse $5 * 0.042$

Distanz zu Bauland nach Alter und

$+\quad$ Geschlecht Klasse $6 * 0,050$

$+\quad$ Distanz zu Bauland nach Bildungsgrad Klasse $1 * 0,029$

+ Distanz zu Bauland nach Bildungsgrad Klasse $2 * 0,058$

Distanz zu Bauland nach Bildungs$\operatorname{grad}$ Klasse $3 * 0,088$

+ Distanz Erschließungsstraßen * 0,150

$+\quad$ Dichte MTB- Routen * 0,120

$+\quad$ Dichte Wanderwege * 0,100

$+\quad$ Dichte Forststraßen * 0,080

$+\quad$ Distanz zu Flüssen Klasse $1 * 0,025$

$+\quad$ Distanz zu Flüssen Klasse $2 * 0,017$

$+\quad$ Distanz zu Flüssen Klasse $3 * 0,008$

$+\quad$ Dichte Waldrand * 0,050

$+\quad$ Mittlere Hangneigung zwischen 5 und $50 \% * 0,100$

$=$ „Nutzungswahrscheinlichkeit Mountainbike“"

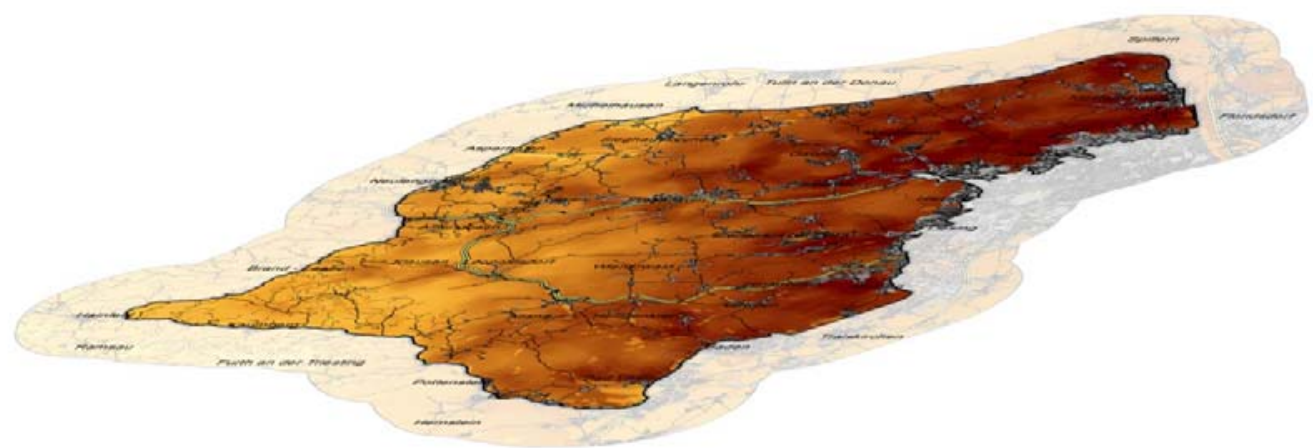

Abbildung 15: Aufsummierung der einzelnen Rasterkarten am Beispiel für das Mountainbiken, (Plangrundlage: BPWW, E.C.O., Land Niederösterreich, www.mtbwienerwald.at, Statistik Austria)

In Abhängigkeit der einzelnen Freizeitaktivitäten kamen sowohl Quell- als auch Zielgebietsparameter mit jeweils unterschiedlichen Gewichtungen zum Einsatz. Die einzelnen Parame- 
ter wurden primär durch Literaturrecherche ausgewählt, teilweise konnte auf die Einschätzung lokaler Experten zurückgegriffen werden. Einzelne Parameter konnten aufgrund fehlender bzw. für den Biosphärenpark Wienerwald nicht flächendeckend vorhandener Daten nicht berücksichtigt werden. Zu diesen zählen beispielsweise die Oberflächenbeschaffenheit der Wege, Aussichtspunkte, Picknickwiesen, Erholungsinfrastruktur wie Spielplätze, TischBankkombinationen, Joggingrouten, Fitnessparcours.

Im Folgenden werden die verwendeten Quell- und Zielgebietsparameter näher erläutert:

\section{Demografische Daten:}

Die demografische Struktur der einzelnen Gemeinden im Untersuchungsgebiet und seinem Umland hat auf die Nutzungswahrscheinlichkeit für die einzelnen Aktivitäten einen hohen Einfluss, da sämtliche Naherholungsaktivitäten eng an das räumliche Umfeld gekoppelt sind.

Die relevanten demografischen Daten wurden mit dem GIS Layer der Baulandumhüllenden auf Ebene der einzelnen Gemeinden verknüpft, um die Einwohnerzahlen, den Bildungsstand sowie das Alter in einen räumlichen Bezug zu setzen. In Abhängigkeit der jeweiligen Erholungsaktivität wurde eine Klasseneinteilung getroffen, um darauf basierend eine unterschiedliche Gewichtung vornehmen zu können.

Die demografischen Daten aus dem Umland des Biosphärenparks wurden bis zu einer Distanz von $12 \mathrm{~km}$ zur Biosphärenparkgrenze in die Modellierung mit einbezogen, da auch für Einwohner von Gemeinden, die keinen Anteil am Biosphärenpark haben, das Naherholungsumfeld im Biosphärenpark liegen kann. Der Pufferbereich von $12 \mathrm{~km}$ wurde unter Berücksichtigung des größten Aktionsradius gewählt. Abbildung 16 zeigt am Beispiel der Klasseneinteilung für das Mountainbiken (Bevölkerung geordnet nach Anzahl der männlichen Bewohner im Alter zwischen 15 und 39 Jahren) die Verknüpfung von demographischen Daten mit dem Layer der Baulandumhüllenden auf Gemeindeebene. 


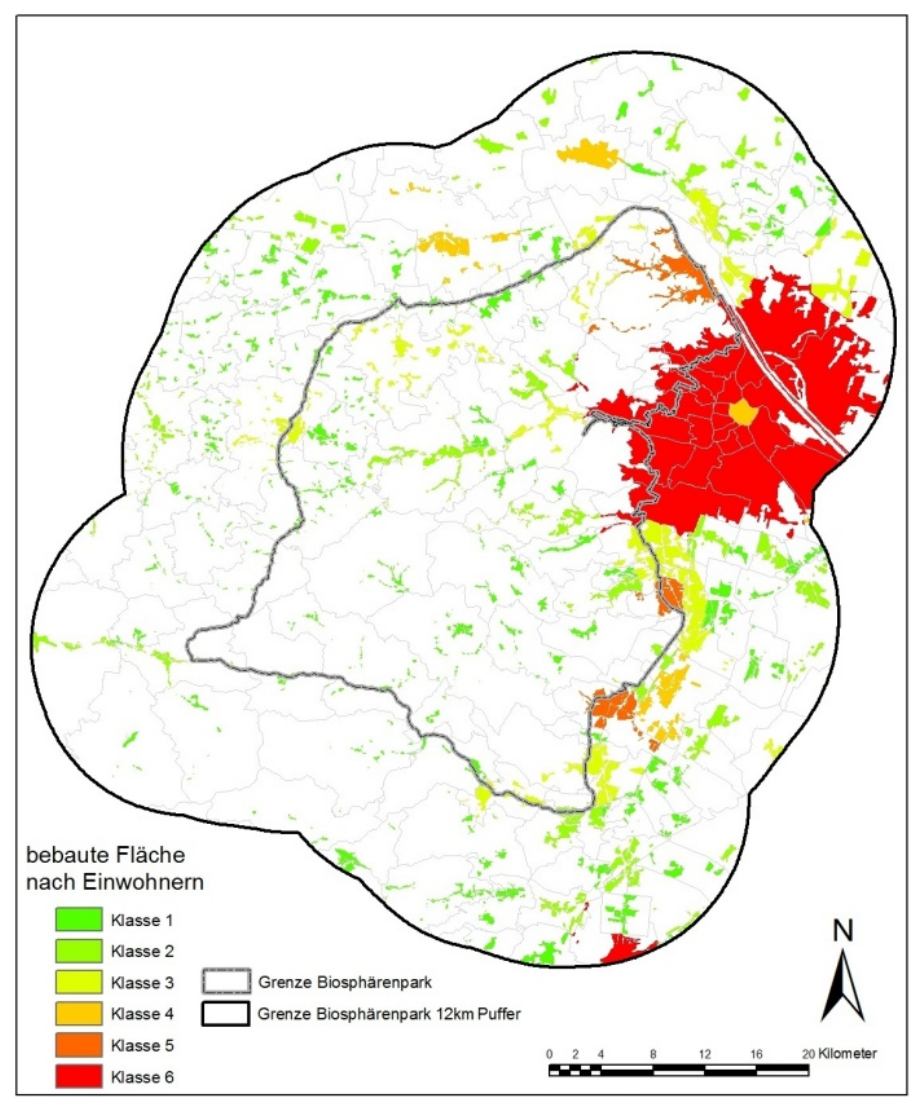

Abbildung 16: Beispiel für die Verknüpfung von demographischen Daten mit der Baulandumhüllenden auf Gemeindeebene, (Plangrundlage: BPWW, Land Niederösterreich, Stadt Wien, Statistik Austria).

\section{Hangneigung:}

Die Hangneigung (durchschnittliche Steigung) stellt einen limitierenden Faktor bei der Ausübung von Freizeitaktivitäten dar. In Abhängigkeit von der Aktivität können sowohl eine zu geringe als auch eine zu hohe Hangneigung einen limitierenden Faktor darstellen.

Die Hangneigung wurde aus dem digitalen Geländehöhenmodell berechnet, welches in Form einer Rasterkarte mit einer Auflösung von $25 \times 25$ m pro Rasterzelle für jede Zelle die Höhe (m. über Adria) angibt. Aus dem Höhenunterschied von benachbarten Rasterzellen konnte direkt die Hangneigung berechnet werden. Für die Berücksichtigung der Hangneigung als Einflussgröße für die verschiedenen Indikatoraktiviäten musste berücksichtigt werden, dass aus der durchschnittlichen Neigung einer Fläche nicht direkt auf die Neigung eines darauf verlaufenden Weges geschlossen werden kann. Daher wurden die Grenzwerte für die Hangneigung als limitierenden Faktor großzügig gewählt.

Abbildung 17 zeigt die Hangneigung im Biosphärenpark in Prozent. 


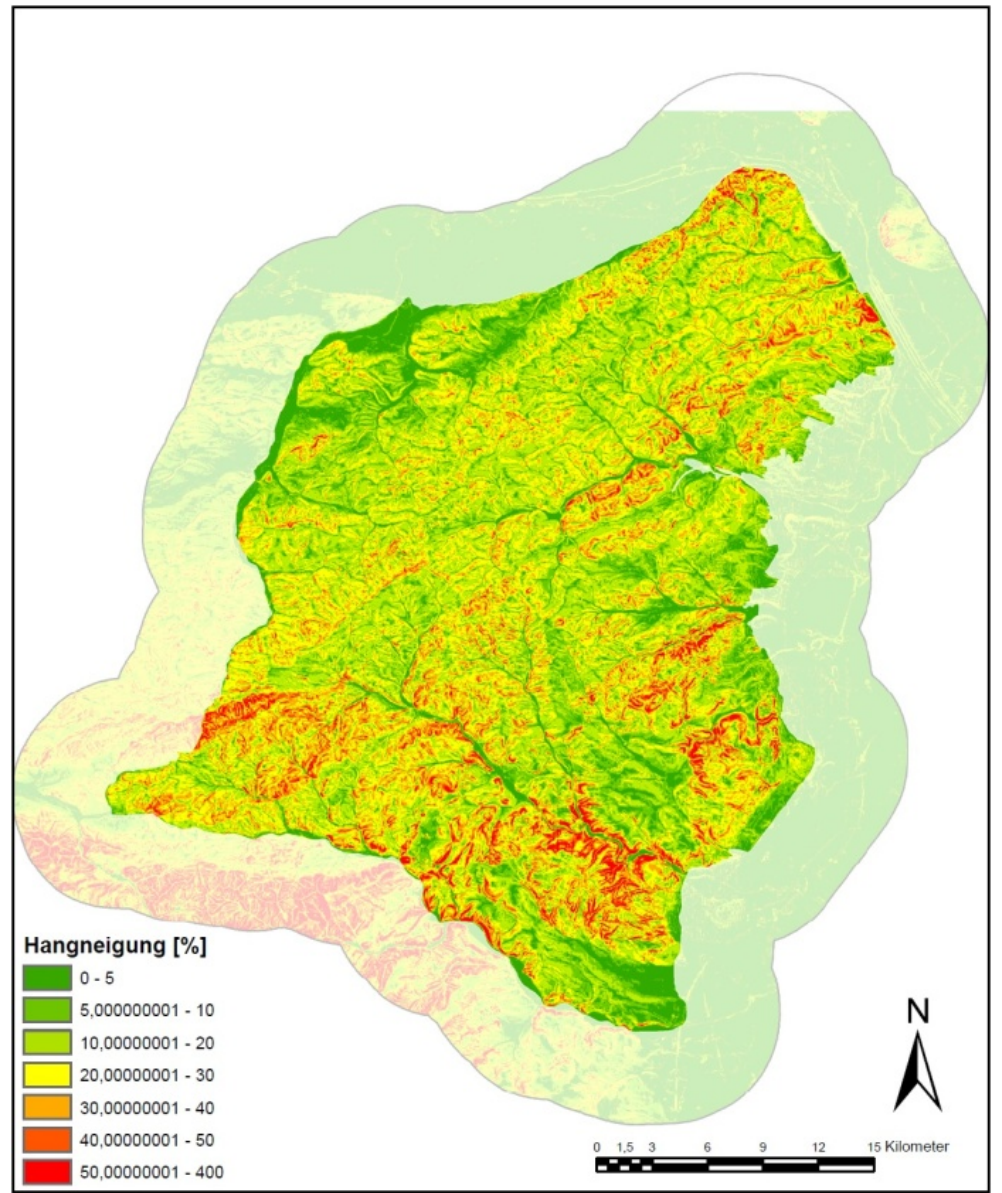

Abbildung 17: Hangneigung in Prozent (Plangrundlage: BPWW, E.C.O.)

\section{Aktionsradius:}

In Abhängigkeit der einzelnen Aktivitäten ist die durchschnittlich zurückgelegte Strecke unterschiedlich groß. Bei Aktivitäten mit höheren Geschwindigkeiten (Ballonfahren, Mountainbiken) ist der Aktionsradius naturgemäß höher als bei Aktivitäten mit langsamerer Fortbewegungsgeschwindigkeit (Aktivität mit Hund, Geocachen, Joggen).

Für die Berechnung des durchschnittlichen Aktionsradius wurde daher für jede Aktivität eine durchschnittliche Streckenlänge auf Basis vorhandener Routen ermittelt. Dabei wurde angenommen, dass eine Strecke im Regelfall in Form einer Kreislinie zurückgelegt wird. Der Aktionsradius entspricht demnach dem Durchmesser eines Kreises mit dem Umfang der jeweiligen durchschnittlichen Streckenlänge der betrachteten Aktivität. Es kann nicht angenommen werden, dass nach dem Überschreiten des Aktionsradius kein Nutzer aus dem jeweiligen Gebiet anzugreffen ist. Die Nutzungswahrscheinlichkeit ist deshalb außerhalb des Aktionsradius nicht null, sondern nimmt nur in einem stärkeren Intervall kontinuierlich ab.

\section{Landschaftsattraktivität:}

Die Landschaftsattraktivität für Erholungssuchende wird durch eine Vielzahl von Faktoren beeinflusst. In die Modellierung der Nutzungswahrscheinlichkeit wurden die Dichte an $\mathrm{Ge}-$ wässern sowie die Grenzliniendichte der Waldränder miteinbezogen. Auf den Flächen, bei denen die Grenzliniendichte dieser Landschaftselemente größer ist, ist der Wald mehr frag- 
mentiert - Waldgebiete und offene Flächen wechseln sich also häufiger ab - als auf jenen Flächen auf denen eine geringere Grenzliniendichte vorhanden ist.

Für die Berechnung der Grenzliniendichte wird die Linienlänge aller Linien (im vorliegenden Fall des Waldrandes) in einem definierten Suchradius $(1 \mathrm{~km})$ um die zu berechnende Rasterzelle aufsummiert, und durch die Fläche des Kreises, welcher sich durch den Suchradius ergibt, dividiert. Abbildung 18 zeigt die Grenzliniendichte des Waldes. Bei den dunkleren Bereichen ist die Grenzliniendichte größer als an den hell dargestellten Bereichen.

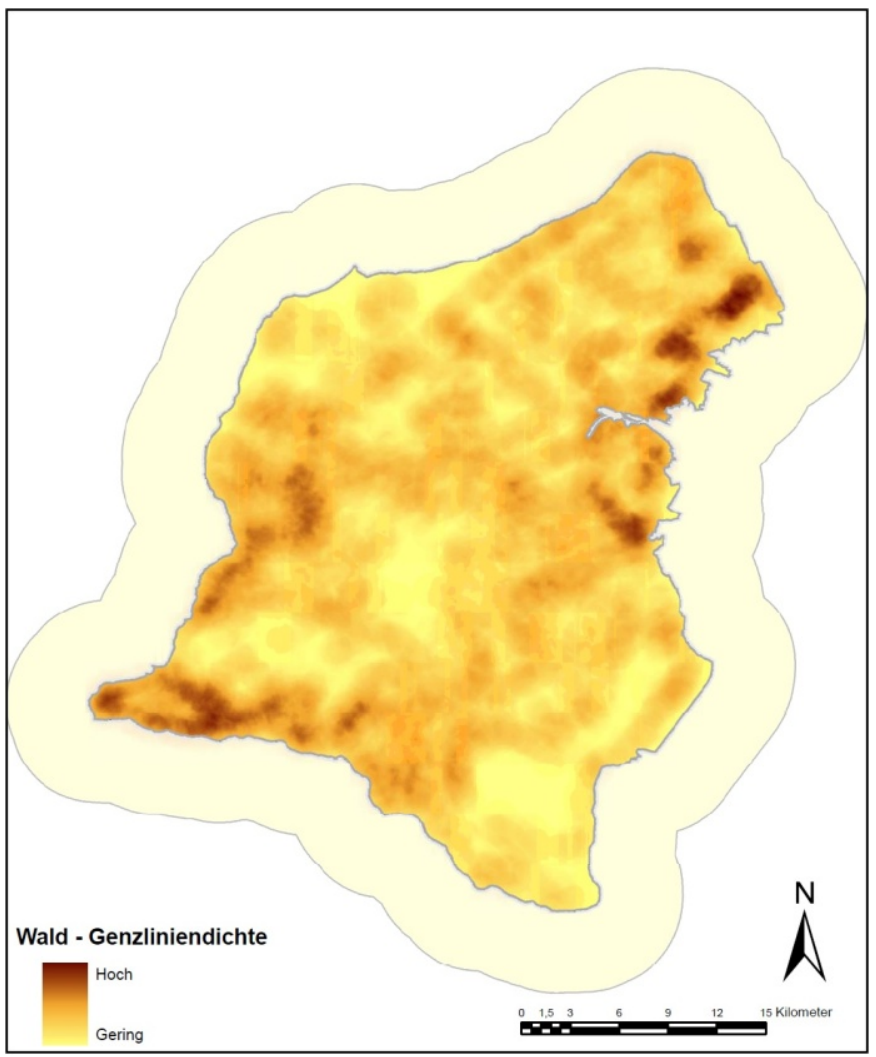

Abbildung 18: Grenzliniendichte Wald (Plangrundlage: BPWW, E.C.O.)

\section{Wegenetz und Wegedichte:}

Bei der Erschließung werden sowohl speziell ausgewiesene Wege für die einzelnen Nutzergruppen (z.B. Mountainbikerouten), generelle Wanderwege sowie Forststraßen mit unterschiedlichen Gewichtungen, Erschließungsstraßen und unbefestigte Wege mit berücksichtigt. Je höher die Dichte der einzelnen Wege ist, umso höher wird die Erschließung und somit die Attraktivität des Gebiets für Erholungssuchende beurteilt. Die Vorgangsweise zur Berechnung der Wegedichte erfolgte analog jener bei der Grenzliniendichte des Waldrandes.

Abbildung 19 zeigt das Wegenetz der Mountainbikerouten, der Wanderwege und der Forststraßen. 


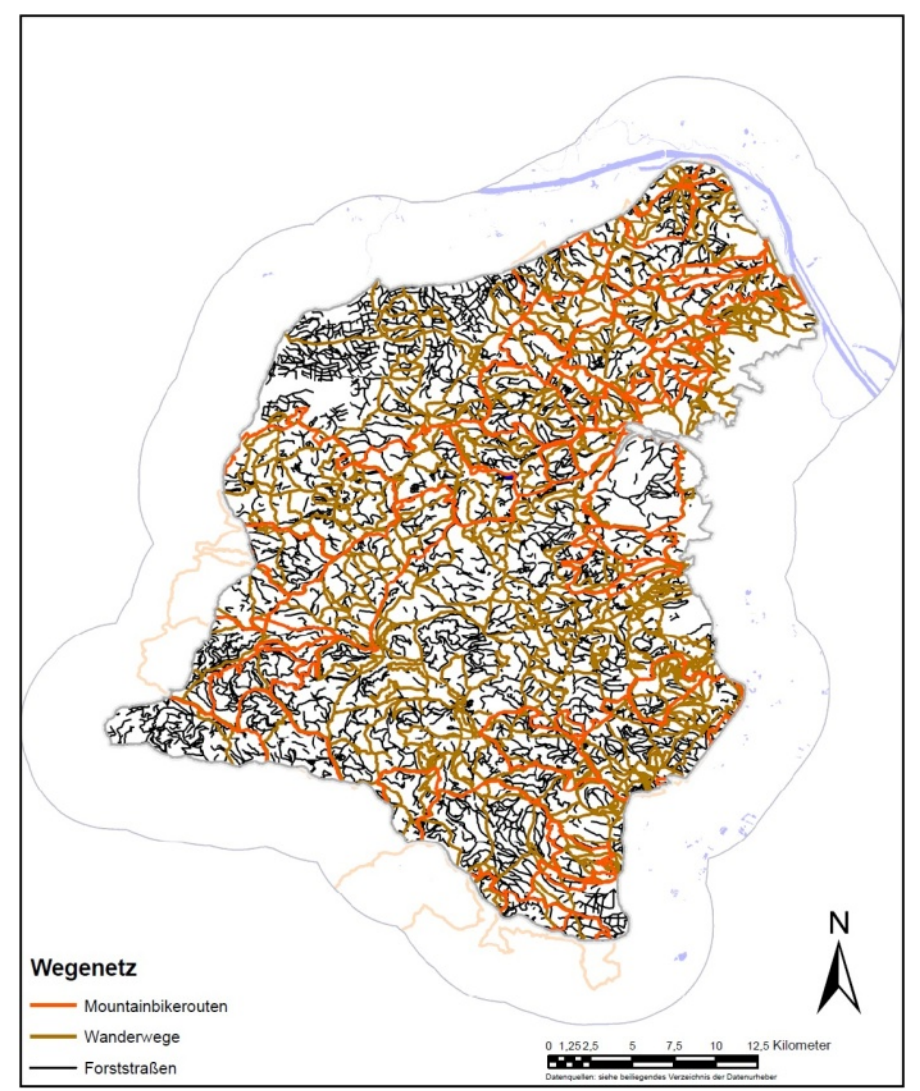

Abbildung 19: Wegenetz (MTB-Routen, Wanderwege, Forststraßen) (Plangrundlage: BPWW, E.C.O., www.mtbwienerwald.at)

\section{Geocache Standorte:}

Die Suche nach Geocaches ist nur dort möglich, wo bereits Geocaches von anderen Geocachern versteckt wurden. Die Lage der Geocaches spielt somit eine wesentliche Rolle für die Modellierung der Nutzungswahrscheinlichkeit. Für die Modellierung wurde auf die verorteten Standorte der Austrian Geocache Database (www.aj-gps.net, Zugriff am 06.09.2010) zurückgegriffen, welche unter anderem die Daten des für Österreich meist genutzten Portal www.geocaching.com verarbeiten.

Abbildung 20 zeigt die Verteilung der Geocaches im Biosphärenpark und dessen Umgebung. Es ist in der Abbildung gut zu erkennen, dass sich der Großteil der Geocaches im urbanen bzw. in dessen Nähe befindet. 


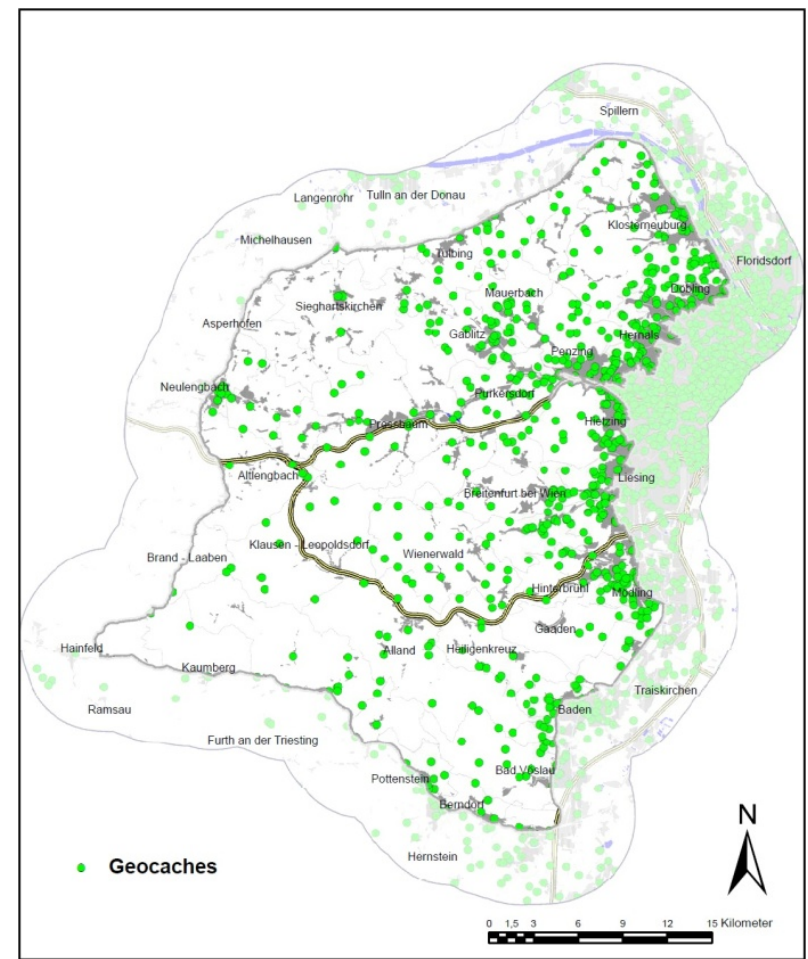

Abbildung 20: Geocaches (Plangrundlage: BPWW, Land Niederösterreich, www.aj-gps.net)

\section{Registrierte Hunde:}

Die Anzahl der Hunde pro Gemeinde ist für Aktivitäten mit Hund ein wesentlicher Faktor. Die Anzahl der registrierten Hunde wurde über die Heimtierdatenbank (Niederösterreich) bzw. über die Magistratsabteilung 6 (Wien) erhoben, wo Daten für das Jahr 2008 verfügbar waren (www.wien.gv.at/statistik). Sowohl in Wien als auch in Niederösterreich ist die Zahl tatsächlich vorkommender Hunde jedoch deutlich höher als jene der registrierten Hunde - genauere Angaben sind hierzu leider nicht möglich.

Abbildung 21 gibt einen Überblick über die Anzahl der registrierten Hunde pro Gemeinde. 


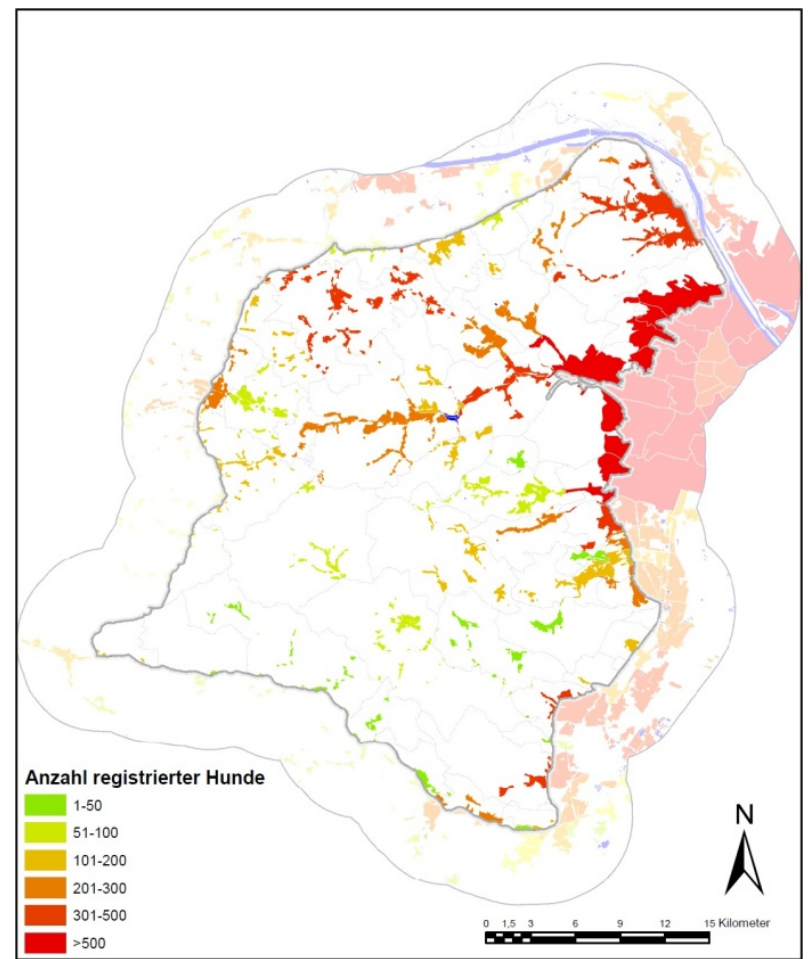

Abbildung 21: Anzahl registrierter Hunde (Plangrundlage: BPWW, Land Niederösterreich, Stadt Wien)

\section{Windrichtung:}

Die vorherrschenden Windrichtungen spielen für die räumliche Verteilung bzw. Nutzungswahrscheinlichkeit der Ballonfahrer eine entscheidende Rolle. Jene wurden auf der Webseite der Zentralanstalt für Meteorologie und Geodynamik (www.zamg.ac.at) ermittelt. Dort sind von vier Messstationen, welche sich im Wienerwald befinden, langjährige Mittelwerte (19712000) der Verteilung der Windrichtungen verfügbar. Aus der mittleren Verteilung der Windrichtung wurde die Wahrscheinlichkeit abgeleitet, in welche Richtung sich ein Heißluftballon nach dem Start bewegt. Wie in Kapitel xx beschrieben, können Ballonfahrer sowohl von fixen, für die Luftfahrt ausgewiesenen Startplätzen als auch von sonstigen für den Einzelfall genehmigten Standorten starten. Da die Mehrheit der Starts von den ausgewiesenen Startplätzen durchgeführt wird, wird die Nutzungswahrscheinlichkeit für das Ballonfahren von diesen ausgehend modelliert (Abbildung 22). 


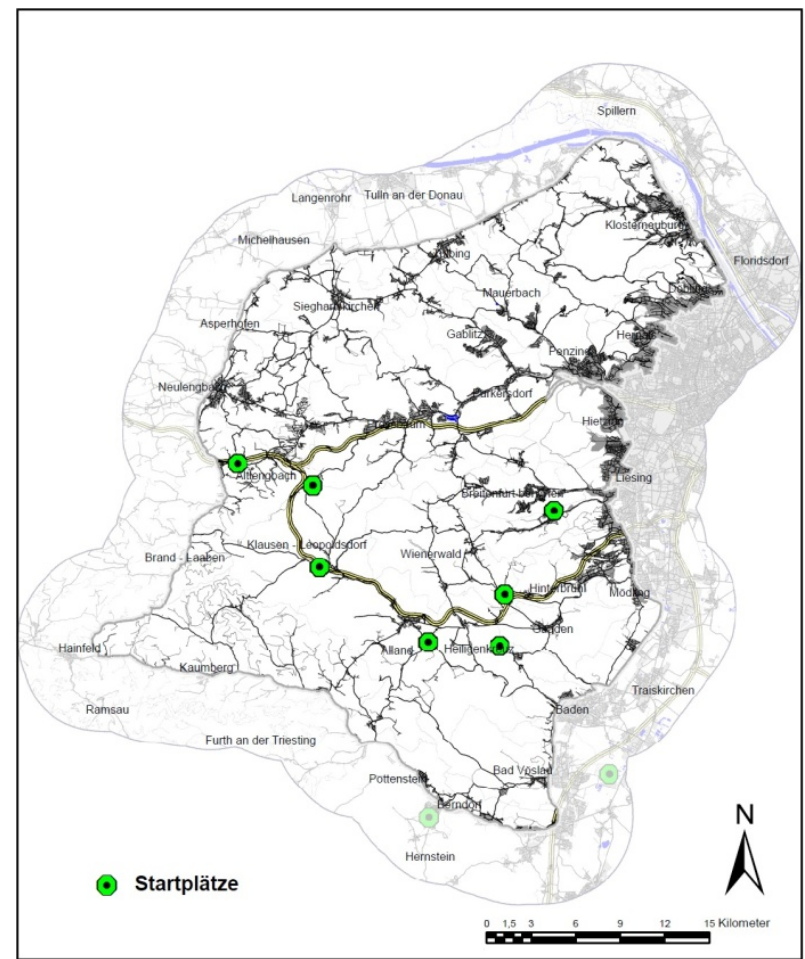

Abbildung 22: Startplätze für Heißluftballone (Plan-grundlage: BPWW, Land Niederösterreich, Stadt Wien)

In 3.4.1 werden die verwendeten Parameter sowie die spezifischen Gewichtungen für die einzelnen Aktivitäten vorgestellt. 


\subsubsection{Indikator-Wildtierarten}

\subsubsection{Auswahl der Indikatorarten}

Analog zu den Indikatoraktivitäten wurden fünf Wildtierarten ausgewählt, die stellvertretend für die im Untersuchungsgebiet vorkommenden Artengruppen im gegenständlichen Projekt näher untersucht wurden.

Die Funktion von Indikatorarten innerhalb des Projekts besteht darin, Wechselwirkungen zwischen dem Einfluss von Landnutzungsansprüchen am Beispiel von Freizeitaktivitäten und der Sensitivität des Naturraums anzuzeigen. Die wesentlichste Anforderung an die Auswahl der Indikatorarten bildete das Vorhandensein von räumlichem und zeitlichem Konfliktpotenzial mit den relevanten Indikatoraktivitäten. Das heißt, von Indikator-Aktivitäten soll eine signifikante Störwirkung auf Indikator-Arten ausgehen, und Indikator-Arten sollen erhöhte Störungssensibilität gegenüber Indikator-Aktivitäten aufweisen. Beides erfordert räumlichzeitliche Überlagerungen in denselben Teilräumen des Biosphärenparks.

Darüber hinaus wurden folgende weitere konkrete Auswahlkriterien für Indikator-Arten definiert:

- Arten mit hohem und vergleichsweise geringem Raumbedarf

- Abhängigkeit von guter Landschafts-Konnektivität (einschließlich zu Populationen / Lebensräumen außerhalb des Biosphärenparks)

- Repräsentativität für charakteristische Habitattypen im Wienerwald

- Schirmfunktion für weitere Arten mit ähnlichen Lebensraumansprüchen ("umbrella species")

- Häufige und seltene Arten

- Jagdbare und geschützte bzw. geschonte Arten

- Säugetierarten und Vogelarten

- Charismatische Arten ("flagship species")

- Arten mit hohem Erlebniswert für Erholungssuchende und Biosphärenparkbewohner

- Ausreichende Datenverfügbarkeit.

Ein Leitprinzip bei der Anwendung der Auswahlkriterien war es, ein Artenensemble zu erhalten, das im Sinne des Schirmartenansatzes ein möglichst breites Spektrum der im Biosphärenpark vorkommenden Tierarten abdeckt.

Der Fokus lag dabei ausschließlich um Vogel- und Säugetierarten. Den Untersuchungsansatz auf Insekten oder Reptilien bzw. Amphibien auszuweiten, hätte den Rahmen des Projekts gesprengt. Ansatzweise wird aber deren im Vergleich zu Vögeln und Säugetieren kleinräumige Lebensweise durch die Behandlung einer nur lokal vorkommenden Tierart (dem Ziesel) berücksichtigt. Auf biologische Spezifika von Insekten und Reptilien konnte dabei aber nicht eingegangen werden. Aspekte wie Verinselung, fehlende Ausweichhabitate oder eingeschränkte Mobilität, die auch die letztgenannten Artengruppen betreffen, wurden behandelt. 
Gemeinsam mit den Stakeholdern wurden folgende Indikatorarten festgelegt:

- Rothirsch (Rotwild) (Cervus elaphus)

- Wildschwein (Schwarzwild) (Sus scrofa)

- Auerhuhn (Auerwild) (Tetrao urogallus)

- Schwarzstorch (Ciconia nigra)

- Ziesel (Spermohillus citellus)

Die getroffene Auswahl an Indikatorarten soll stellvertretend für eine Reihe von Tierarten stehen, die die Lebensräume im BP Wienerwald nutzen und prägen. Es wurden vor allem Arten mit starkem Bezug zu Waldbiotoptypen ausgewählt, da dies die häufigen und dominanten Lebensräume im Biosphärenpark sind. Mit Ausnahme des Ziesels wurde bewusst auf die Behandlung von reinen Offenlandarten, die im Biosphärenpark besonders im Südosten entlang der Thermenlinie, aber auch im nordwestlichen „Wiesenwienerwald“ wichtige Merkmale der Vielfalt darstellen, verzichtet. Für diese Arten sind andere Konfliktsituationen zu betrachten und Analysen notwendig, die in diesem Projekt keinen Platz finden konnten. Oftmals steht in diesen Lebensraumtypen z.B. weniger die Freizeitnutzung als Konfliktpartner im Mittelpunkt, sondern Konfliktpotenziale entstehen eher mit der landwirtschaftlichen Nutzung.

\section{Rotwild}

Diese Säugetierart kommt vor allem im südlichen Teil des Biosphärenparks Wienerwald vor, wird bejagt und ist sensibel sehr gegenüber menschlichen Störungen. Rotwild wählt seinen Einstand stark nach dem Konzept „Sicherheit vor Nahrung“. Daraus können wirtschaftliche Probleme entstehen, wenn das Wild um Störungen auszuweichen seinen Einstand in Schäloder verbissgefährdete Waldbestände verlegt. Obwohl die Art ganzjährig im Biosphärenpark vorkommt besteht eine ausgeprägte Saisonalität was die Lebensraumansprüche, Störungsempfindlichkeit und damit Managementimplikationen anlangt. Die Datenlage zu der Art hinsichtlich Biologie, Lebensraumnutzung im Biosphärenpark, sowie Management und Jagd ist sehr gut.

\section{Wildschwein}

Wildschweine kommen im gesamten Biosphärenpark in unterschiedlicher Dichte vor und werden bejagt. Durch die schwierige Bejagbarkeit und das hohe Vermehrungspotential, bei gleichzeitig hoher Anpassungsfähigkeit an Störungen, ergibt sich eine vielschichtige Problemsituation, die auch im Biosphärenpark Wienerwald zum Tragen kommt. Ein Einfluss auf das Ausbreitungspotential von Auerwild und anderen seltenen Bodenbrütern im Biosphärenpark wird ebenfalls angenommen. Weitere Auswahlkriterien umfassten die wirtschaftliche Relevanz (landwirtschaftliche Schäden und jagdlicher Wert), die gute Datenverfügbarkeit sowie eine potentielle Gefährlichkeit für den Menschen, vor allem im Vergleich zu den anderen untersuchten Arten. Außerdem ist bei dieser Art das Wirkungsgefüge aus einem anderen Blickwinkel zu betrachten. Hier üben die Aktivitäten der Freizeitnutzung einen größeren Einfluss auf das Management dieser Tierart (Raumnutzung, Bejagbarkeit, Schadensvermeidung) aus als auf die Tierart selbst. 


\section{Auerhuhn}

Diese Vogelart wird in Österreich bejagt, im Biosphärenpark wurden allerdings in letzter Zeit keine Abschusse mehr getätigt. Es ist eine nicht ziehende, bodenbrütende Vogelart, die in Mitteleuropa vor allem mit Lebensraumverlust und menschliche Aktivitäten zu kämpfen hat. Die Bestandessituation in Niederösterreich wird als stabil beschrieben (Wildauer et al., 2008). Auerhühner sind im Biosphärenpark vereinzelt nachgewiesen. Es handelt sich dabei höchst-wahrscheinlich um ein Ausstrahlen des südwestlich des Biosphärenparks im Bereich Guten-stein gelegenen. Auerwildvorkommens. Folgende Aspekte waren relevant für die Auswahl dieser Art. Sie ist jagdbar und gleichzeitig von hohem Naturschutzinteresse. Aufgrund der Bestandessituation und der angenommenen räumlichen Verteilung im Rahmen eines Meta-populationskonzepts ist diese Art auf erhöhte Konnektivität angewiesen. Die Einzelnachweise im Biosphärenpark können als Hinweise auf Lebensraumpotential gesehen werden, und der Biosphärenpark als Lebensraum und Managementeinheit wäre geeignet einen positiven Beitrag zur natürlichen Wiederausbreitung zu leisten. Des Weiteren ist die Informationslage zu Lebensraumbedürfnissen und Störungssensibilität dieser Art sehr gut. Durch die Art der Jungenaufzucht (Bodenbrüter) wurde es allerdings notwendig das Wildschwein mit in den Artenkatalog aufzunehmen, um eine realistische Abbildung der Situation zu ermöglichen.

\section{Schwarzstorch}

Diese Vogelart ist nicht jagdbar. Der Schwarzstorch ist ein Sommergast, der die Region des Biosphärenparks zur Jungenaufzucht nutzt. Er ist von hohem Naturschutzinteresse und die Ansprüche großräumig agierender Arten werden durch die Einbeziehung dieser Art berücksichtigt. Der Schwarzstorch stellt sehr spezifische Anforderungen an sein Brutrevier, bezüglich Nahrungsangebot, Horstbäume, Waldstruktur und Störungssituation. Durch seine Lebensweise ist er auch repräsentativ für Altbestände und Kernzonen im Biosphärenpark. Im Vergleich zu anderen Zugvogelarten ist beim Schwarzstorch die Datenlage als günstig zu beurteilen.

\section{Ziesel}

Diese Säugetierart ist nicht jagdbar und von hohem Naturschutzinteresse bei guter Datenverfügbarkeit. Ziesel kommen im Biosphärenpark nur auf der Perchtoldsdorfer Heide vor. Dieser Trockenrasenlebensraum ist sehr speziell. Damit steht das Ziesel für eine Artengruppe von Tieren, die eine geringe Raumnutzung und Ausbreitungstendenz im Biosphärenpark zeigt. Während bei großräumig, am Boden agierenden Arten die Konnektivität des Lebensraums auf regionaler Ebene ein wichtiger Punkt ist, stehen hier konkrete Flächen, die erhalten bleiben müssen im Vordergrund.

Eine literaturgestützte, vertiefende Betrachtung der einzelnen Indikatoraktivitäten folgt in Kapitel 3.2.1.

Eine Übersicht zur Charakterisierung der ausgewählten Indikatorarten hinsichtlich projektrelevanter Aspekte ist der nachstehenden Tabelle 7 zu entnehmen. 
Tabelle 7: Charakterisierung der Indikatorarten hinsichtlich projektrelevanter Kriterien.

\begin{tabular}{|c|c|c|c|c|c|c|}
\hline $\begin{array}{l}\text { Indikator- } \\
\text { Arten }\end{array}$ & $\begin{array}{l}\text { Indikator-Erholungsaktivitäten } \\
\text { (mit potenziell hoher } \\
\text { Störungsintensität) }\end{array}$ & Störungsmechanismus & indizierte Konfliktaspekte & betroffene Interessen & Erfüllung Auswahlkriterien & Schirmart für \\
\hline Rothirsch & $\begin{array}{l}\text { - Aktivitāten mit Hunden } \\
\text { - Geocaching } \\
\text { - Mountainbiking (Tag/ } \\
\text { Nacht/faktisch ganzjährig) } \\
\text { - Jogging (Tag/Nacht/ganzjährig) }\end{array}$ & 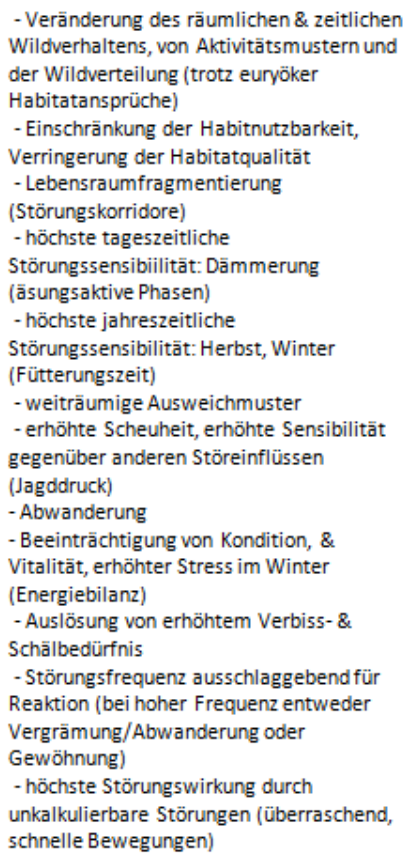 & $\begin{array}{l}\text { - Wildschäden in Forst- \& } \\
\text { Landwirtschaft } \\
\text { - Bejagbarkeit } \\
\text { - - Jagdowert } \\
\text { - Uberwinterung } \\
\text { (Fütterungsstandorte etc.) } \\
\text { - Lebensraumzerschneidung / } \\
\text { Barrieren: } \\
\text { Beeinträchtigung } \\
\text { Wanderungs- } \\
\text { \&Wechselverhalten } \\
\text { - regionale Verbeitung von } \\
\text { Rothirschpopulationen }\end{array}$ & $\begin{array}{l}\text { - Erholungsnutzer: Beobachtbarkeit } \\
\text { - Naturschutz: Erhaltung regionaler } \\
\text { Populationen, ungestörte } \\
\text { Waldentwicklung in Kernzonen } \\
\text { - Jagd: attraktives } \\
\text { Wildartenspektrum, jagdlich } \\
\text { nutzbare Bestandesgrößße, } \\
\text { Abschussplanerfüllung, } \\
\text { Fütterungsbetrieb } \\
\text { - Grundeigentümer, Verpächter: } \\
\text { Jagdwert } \\
\text { - Waldeigentümer, - bewirtschafter: } \\
\text { Verjüngung, forstlicher Ertrag, } \\
\text { Kosten }\end{array}$ & $\begin{array}{l}\text { - jagdbar } \\
\text { - hoher Raumbedarf, großrrāumig } \\
\text { agierend } \\
\text { - ermöglicht Einbeziehung der } \\
\text { Landschaftskonnektivitāt } \\
\text { - umbrella species } \\
\text { - besiedelt typische } \\
\text { Lebensraumtypen im BPWW } \\
\text { - störungsempfindlich } \\
\text { - (vergleichsweise) häufig } \\
\text { - Sāugetierart } \\
\text { - charismatische "flagship" Art } \\
\text { - gute Datenverfügarirkit } \\
\text { - Konfliktpotenzial (Wildschaden) } \\
\text { mit wirtschaftlichen } \\
\text { Landnutzungsansprüchen }\end{array}$ & $\begin{array}{l}\text { - Wildlebende } \\
\text { Wiederkäuer } \\
\text { - Großsprädatoren }\end{array}$ \\
\hline Wildschwein & $\begin{array}{l}\text { - Aktivitāten mit Hunden } \\
\text { - Geocaching } \\
\text { - Mountainbiking (Tag/ } \\
\text { Nacht/faktisch ganzjärig) } \\
\text { - Jogging (Tag/Nacht/ganzjährig) }\end{array}$ & 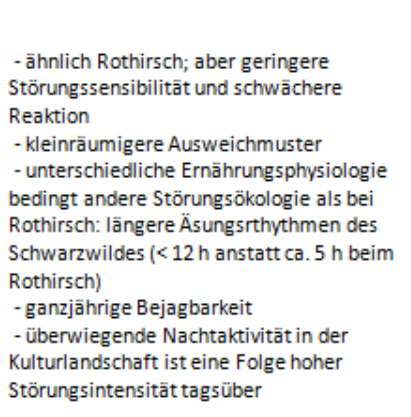 & $\begin{array}{l}\text { - Landwirtschaftliche } \\
\text { WWilschäden (Fraßschäden, } \\
\text { Wiesenumbruch) } \\
\text { - "ökologischer Wildschaden": } \\
\text { Bodenbrüter; Trockenrasen, } \\
\text { Orchideenwiesen u.a. } \\
\text { naturschutzacfachlich wertvolle } \\
\text { Grünlandbiotope } \\
\text { - Bejagbarkeit (Unterschied zu } \\
\text { Rotwild: Bejagung auch nachts) } \\
\text { - Jagdwert } \\
\text { - Barrieren, } \\
\text { Wanderungsverhalten } \\
\text { - Angriffsrisiko gegenüber } \\
\text { Erholungssuchenden }\end{array}$ & $\begin{array}{l}\text { - Erholung snutzer: Beobachtbarkeit } \\
\text { - Grundeigentümer, Verpāchter: } \\
\text { Jagdwert } \\
\text { - Landwirte: Schadensvermeidung, } \\
\text { Minimierung Kosten für } \\
\text { SchutzmaßBnahmen, } \\
\text { Wildschadensabgeltungen } \\
\text {-Grundeigentümer, Verpächter: } \\
\text { Jagdwert } \\
\text { - Jagd: attraktives } \\
\text { Wildartenspektrum, } \\
\text { Bejagungseffizienz } \\
\text { - Naturschutz: Erhaltung } \\
\text { Grünlandbiotope, seltene/ } \\
\text { gefährdete Bodenbrüter }\end{array}$ & \begin{tabular}{|l} 
- jagdbar \\
- Konfliktpotenzial (Wildschaden) \\
mit wirtschaftlichen \\
Landnutzungsansprüchen \\
- großßräumiger Aktionsradius \\
(Fernwechsel) \\
- besiedelt typische \\
Lebensraumtypen im BPWW \\
- (vergleichsweise) \\
störungsempfindlich \\
- hāuffig, großräumige \\
Verbreitung \\
- Sāugetierart \\
- Datenverfügbarkeit
\end{tabular} & \\
\hline
\end{tabular}

IESP - Towards Integrated Ecological Spatial Planning for the Wienerwald Biosphere Reserve 


\begin{tabular}{|c|c|c|c|c|c|c|}
\hline $\begin{array}{l}\text { Indikator- } \\
\text { Arten }\end{array}$ & \begin{tabular}{|l} 
Indikator-Erholungsaktivitäten \\
(mit potenziell hoher \\
Störungsintensität)
\end{tabular} & Störungsmechanismus & indizierte Konfliktaspekte & betroffene Interessen & Erfüllung Auswahlkriterien & Schirmart für \\
\hline Auerhuhn & $\begin{array}{l}\text { - Aktivitāten mit Hunden - } \\
\text { Geocaching - Mountainbiking (Tag/ } \\
\text { Nacht/faktisch ganzjährig) - Jogging } \\
\text { (Tag/Nacht/ganzjährig) }\end{array}$ & 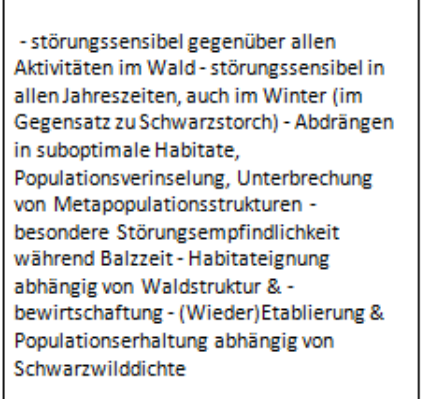 & $\begin{array}{l}\text { - interspezifische Konkurrenz } \\
\text { durch Schwarrwild- } \\
\text { Fragmentierung / Barrieren: } \\
\text { Vernetzung mit Quellgebieten \& } \\
\text { Populationsaustausch } \\
\text { notwendig - Habitateignung } \\
\text { hängt von Waldstruktur und } \\
\text { forstlicher } \\
\text { Bestandesbehandlung ab - } \\
\text { geeignete Habitate mit hoher } \\
\text { Biotopqualität sind gleichzeitig } \\
\text { Gebiete mit hoher } \\
\text { Erholungsattraktivitāt } \\
\text { (aufgelichtete Waldbestānde) }\end{array}$ & $\begin{array}{l}\text { - Naturschutz, Artenschutz: } \\
\text { Schutzgut Vogelschutz-RL, seltene \& } \\
\text { gefährdete Art - Jagd: attraktives } \\
\text { Wildartenspektrum - } \\
\text { Grundeigentümer, Verpächter: } \\
\text { Jagdwert - Wiedereinbürgerung im } \\
\text { BPWW angestrebt }\end{array}$ & $\begin{array}{l}\text { - jagdbar - Vogelart - selten, } \\
\text { gefăhrdet, geschüttzt - auf } \\
\text { großrräumige Konnektivität } \\
\text { angewiesen (Metapopulationen) - } \\
\text { Datenverfügbarkeit: } \\
\text { Lebensraumbeürnisse \& } \\
\text { Störungssensibilitāt gut } \\
\text { untersucht - charismatische Art }\end{array}$ & $\begin{array}{l}\text { - Bodenbrüter - } \\
\text { Spechtarten - } \\
\text { Lebens- } \\
\text { gemeinschaften } \\
\text { lichter } \\
\text { Altholzwälder } \\
\text { (insb. } \\
\text { Höhlenbrüter) - } \\
\text { Baummarder }\end{array}$ \\
\hline Schwarzstorch & $\begin{array}{l}\text { Störung durch alle menschl. } \\
\text { Altivitäten in Horstnähe, teils auch } \\
\text { in Nahrungsräumen, einschließllich: } \\
\text { - Mountainbiking } \\
\text { - Jogging } \\
\text { - Aktivitäten mit Hunden } \\
\text { - Geocaching } \\
\text { - Lagern, Picknicken }\end{array}$ & 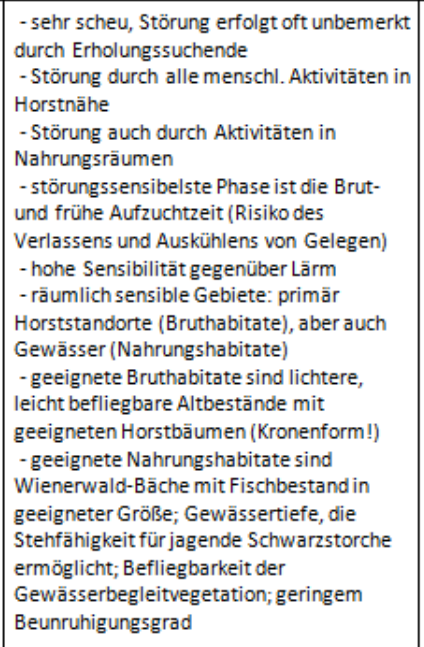 & $\begin{array}{l} \\
\text { - Erhaltung von } \\
\text { (Brut)populationen }\end{array}$ & - Naturschutz, Artenschutz & $\begin{array}{l}\text { - nicht jagabar } \\
\text { - repräsentativ für Altbestānde } \\
\text { (u.a. Kerrnzonen) } \\
\text {-gute Datenlage } \\
\text { - seltene, geschützte Art } \\
\text { - Vogelart } \\
\text { - grolrärumige Lebensweise } \\
\text { - störungssensibel } \\
\text { - flagship species } \\
\text { - Schirmart }\end{array}$ & $\begin{array}{l} \\
\\
\text { - weitere } \\
\text { waldbewohnende } \\
\text { Vogelarten } \\
\text { - Spechtarten } \\
\text { - - Bewohner lichter } \\
\text { Altholzzälder } \\
\text { (Höhlenbrüter) } \\
\text { - Wald-Greifvögel } \\
\text { - Gemeinschaften } \\
\text { von } \\
\text { feuchtegeprägten/ } \\
\text { Gewässerlebens- } \\
\text { reaumen (Nahrungs- } \\
\text { raum) }\end{array}$ \\
\hline Ziesel & $\begin{array}{l}\text { - Aktivitāten mit Hunden } \\
\text { - Jogging (Tag, Sommer) } \\
\text { - Mountainbiking offroad } \\
\text { - Lagern, Picknicken }\end{array}$ & $\begin{array}{l}\text { - sehr spezifische Habitatansprüche, } \\
\text { Vorkommen an bestimmte Standorte } \\
\text { gebunden } \\
\text { - störungssensibel zu Aktivitätszeiten: bei } \\
\text { Schönweetter, im Sommer, tagsüber }\end{array}$ & \begin{tabular}{|l} 
- Arterhaltung, Erhaltung der \\
Wienerwald-Population \\
- Lebensraumeinschränkung \\
historisch \& generell durch \\
Intensivierung der \\
\end{tabular} & \begin{tabular}{|l|} 
- Erholungssuchende, Öffentlichkeit: \\
Beobachtung, Füttern etc., Ziesel ist \\
für die Perchtolsdorfer Heide DIE \\
charismatische flagship Art \\
- Artenschutzz: geschützte Art (FFH- \\
\end{tabular} & \begin{tabular}{|l|} 
- nicht jagdbar \\
- kleinräumiges, lokal begrenztes \\
Vorkommen mit \\
Einzelflächenmanagement \\
- langfristig auf Konnektivitāt
\end{tabular} & $\begin{array}{l}\text { - stellvertretend } \\
\text { für Offenland } \\
\text { (Magergrünland) } \\
\text { bewohnende Arten } \\
\text { - Feldhamster, }\end{array}$ \\
\hline
\end{tabular}

IESP - Towards Integrated Ecological Spatial Planning for the Wienerwald Biosphere Reserve 


\subsubsection{Angewandte Methoden zur Datenerhebung}

Es wurden folgende Methoden zur Datenerhebung benutzt:

- Jagdgebietsweise Jagdstatistik (Abschuss- und Fallwildzahlen der letzten 5 Jahre)

- Experteninterviews auf Intensivuntersuchungsgebiets- und Biosphärenparkebene

- Erhebung von Schwarzstorchnachweisen (Horstbäume, Sichtungen)

- Verfügbare Karten mit Angaben über Vorkommen der Indikatorarten. 


\subsection{Untersuchungseinheiten}

\subsubsection{Intensivuntersuchungsgebiete}

Abbildung 23 zeigt die Lage der Intensivuntersuchungsgebiete innerhalb des Biosphärenparks. Im Südwesten liegt das Intensivuntersuchungsgebiet Hirschenstein, im Süden das Intensivuntersuchungsgebiet Lindkogel. Im Osten am Wiener Stadtrand liegt das Intensivuntersuchungsgebiet Perchtoldsdorfer Heide. Ursprünglich bestand dieses als einziges Intensivuntersuchungsgebiet aus vier Teilen. Es handelte sich dabei um das Hauptvorkommensgebiet der Ziesel auf der Perchtoldsdorfer Heide selbst, und drei Potentialflächen südlich und westlich davon (Eichkogel und Pfaffstätten bzw. Gumpoldskirchen). Diese Potentialflächen stellten sich im Zuge der ersten Interviews als nicht relevant heraus, da keine Aussetzungen auf diesen Flächen geplant sind und sie für die Tiere auf natürlichem Wege nicht erreichbar sind. Im Nordosten des Biosphärenparks liegt das Intensivuntersuchungsgebiet Sievering im Wiener Teil des Biosphärenparks.

In den Intensivuntersuchungsgebieten wurde auf detaillierter Maßstabebene der erste räumliche Bezug zwischen den Indikatorarten und den Indikatoraktivitäten im Biosphärenpark hergestellt. Die Hauptfunktion der Intensivuntersuchungsgebiete im Projekt bildete die Untersuchung von Konfliktpotenzialen auf lokaler Maßstabsebene, um gemeinsame Konfliktmuster, aber auch spezifische Sondersituationen identifzieren und verallgemeinerbare Konflikttypen ableiten zu können. Die Detailuntersuchungen lieferten wertvolle Einblicke in Konfliktstrukturen und -mechanismen, die sich zum Teil als auf den gesamten Biosphärenpark übertragbar erwiesen. Die gewonnenen Detailinformationen aus den Gebieten erwiesen sich bei der Interpretation der biosphärenparkweiten Ergebnisse als hilfreich. Die in den Intensivuntersuchungsgebieten getesteten und entwickelten Analyse- und Auswertungsmethoden sollten in weiterer Folge auf die Maßstabsebene des gesamten Biosphärenparks übertragen werden. Die Befunde in den Intensivuntersuchungsgebieten dienten als empirische Referenzgrößen, um die bei der Methodenentwicklung zur biosphärenparkweiten GISModellierung der Indikatoraktivitäten verwendeten theoretischen Annahmen und Regeln abzustützen und zu validieren. 


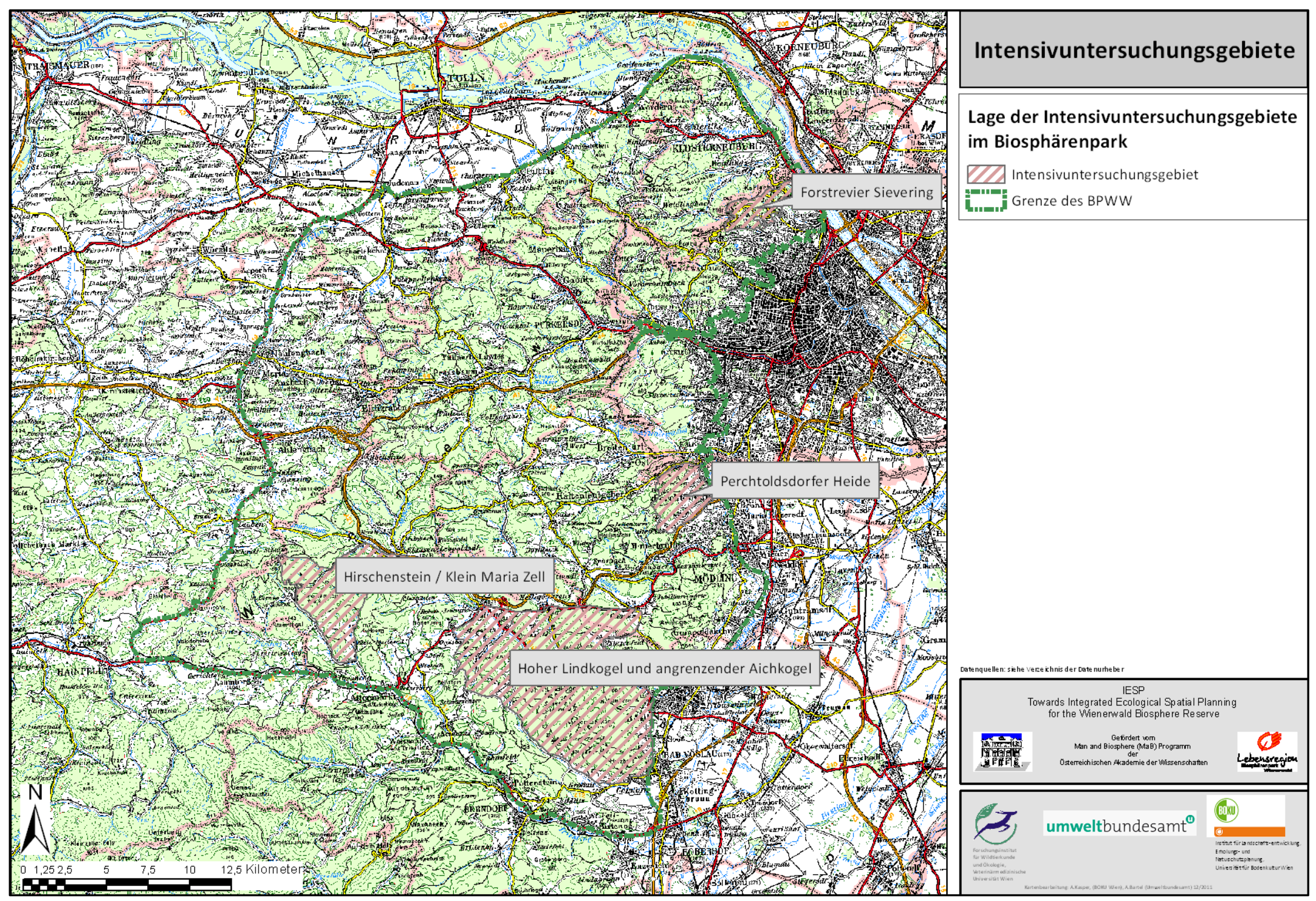

Abbildung 23: Lage der Intensivuntersuchungsgebiete im Biosphärenpark Wienerwald 


\subsubsection{Auswahl der Intensivuntersuchungsgebiete}

Die Auswahl und räumliche Abgrenzung erfolgte nach unterschiedlichen Gesichtspunkten im Rahmen mehrerer Arbeitstreffen der Projekt begleitenden Stakeholdergruppe. Basierend auf den ausgewählten Indikator-Arten und Indikator-Freizeitaktivitäten, den beim 1. Stakeholder Arbeitstreffen gesammelten Informationen sowie vorliegender räumlicher Daten und Einschätzungen zu Schwerpunktgebieten (Vorkommen und Verteilung der Indikatorarten, wie Lage von Horstbäumen des Schwarzstorchs, Lage von rezenten Auerwildsichtungen und historischen Auerwildvorkommen, Rotwildverbreitung etc.; Gebiete mit intensiver und extensiver Erholungsnutzung) wurden vom Projektteam Arbeitskarten mit einem Vorschlag für mögliche Intensiv-Untersuchungsgebiete vorbereitet. In der folgenden Diskussion wurden die Intensiv-Untersuchungsgebiete festgelegt und die Feinabgrenzung mit Farbstift direkt in die Karten eingetragen. Pragmatische bzw. realpolitisch relevante Abgrenzungskriterien, wie Verlauf von Straßenzügen, Gemeindegrenzen und Grundbesitzgrenzen, flossen in die Feinabgrenzung ein. Weiters wurden Grundeigentümer und Ansprech- bzw. Auskunftspersonen je Gebiet identifiziert und die Gebiete im Hinblick auf vorkommende Indikator-Arten und ausgeübte Indikator-Aktivitäten charakterisiert.

In Summe werden die möglichen Kombinationen aus Indikatorarten und Indikatoraktivitäten in den vier ausgewählten Intensivuntersuchungsgebieten vollständig und ausreichend abgedeckt.

\subsubsection{Charakterisierung der Intensivuntersuchungsgebiete}

\section{Hoher Lindkogel}

Grundbesitzer und Auskunftspersonen: ÖBf-AG, Stift Heiligenkreuz, Gemeinde Bad Vöslau, Gemeinde Alland.

Dieses Intensivuntersuchungsgebiet ist geprägt durch mittlere Erholungsnutzung und hohes Wildtiervorkommen. Es kommen alle Indikatorarten bis auf das Ziesel und alle Indikatoraktivitäten in diesem Intensiv-Untersuchungsgebiet vor. Es liegt im Süden des Biosphärenparks, an der sogenannten Thermenlinie nahe den Ortschaften Baden und Bad Vöslau, welche selbst über größere Einwohnerzahlen verfügen und gleichzeitig beliebte Kurorte darstellen. Damit soll der Übergangsbereich zwischen intensiver Freizeitnutzung und hohen Wildtiervorkommen beschrieben werden, da beide Aspekte in diesem Intensivuntersuchungsgebiet in hohem Ausmaß vorkommen. Auch von der Geomorphologie stellt dieses Gebiet ein Übergangsstadium zwischen den flacheren Bereichen im Osten und im Norden des Biosphärenparks und dem „gebirgigeren“ Westen dar, da es sich hier um die letzten Ausläufer der Alpen handelt.

Die beiden Autobahnen A1 und A21 liegen nördlich dieses Intensivuntersuchungsgebiets, wobei die A21 direkt im Norden an das Intensivuntersuchungsgebiet angrenzt.

\section{Hirschenstein / Klein Mariazell}

Grundbesitzer und Auskunftspersonen: ÖBf AG, Forst- und Gutsverwaltung Klein-Mariazell.

Dieses Intensivuntersuchungsgebiet ist geprägt durch geringe Erholungsnutzung und hohes Wildtiervorkommen. Es kommen alle Indikatorarten bis auf das Ziesel und alle Indikatoraktivitäten in diesem Intensiv-Untersuchungsgebiet vor. Es liegt im Westen des Biosphärenparks 
und südwestlich der Autobahn A21. Es gibt keine größeren Ortschaften, die als Quelle für ausgeprägte Naherholung fungieren können. Mit dem Schutzhaus Schöpfl befindet sich allerdings ein weithin bekanntes Ausflugsziel unmittelbar neben dem Intensivuntersuchungsgebiet. Dieses erhöht den Besucherdruck etwas. Ansonsten ist dieses Intensivuntersuchungsgebiet am wenigsten von Freizeitnutzungen betroffen und repräsentiert ein Gebiet mit vergleichsweise ungestörten Wildtiervorkommen. Von allen Intensivuntersuchungsgebieten weist dieses die höchste Reliefenergie auf. Es sind sowohl waldreiche und "gebirgige" Bereiche als auch offene Tieflandbereiche mit Fließgewässern vertreten.

\section{Sievering}

Grundbesitzer und Auskunftspersonen: MA49 Wien.

Dieses Intensivuntersuchungsgebiet ist geprägt durch intensive Erholungsnutzung. Als einzige Indikator-Wildart kommt das Wildschwein vor. Die Dichte der Tiere ist ähnlich den übrigen Biosphärenparkbereichen. Bezüglich des Schwarzwilds gibt es ein sehr enges Nebeneinander mit den Erholungsaktivitäten. Das Schwarzwild weicht bis in Gärten und Siedlungsgebiete (jagdfreie Gebiete) aus. Es kommt oft ungewollt zu „Pseudofütterungen“, in Form von Kompost, Abfall vom Grillen etc. Diese liefern zusätzlichen Besiedlungsanreiz für die Tiere. Ebenso wirken bei Wassermangel Feuchtbiotope anziehend. Die Bejagung durch das Personal der MA49 stellt sich als äußerst schwierig dar und erfolgt vor allem zwischen 4-5 Uhr morgens, oft in Form von Pirschgängen. Zu beachten ist, dass im angrenzenden Waldeigentum des Stifts Klosterneuburg teils unterschiedliche Jagdtechniken angewendet werden, und dass die Freizeitnutzungen schwerpunktmäßig teils zu anderen Zeiten stattfinden.

Durch die unmittelbare Nähe zu Wien, die hervorragende Verkehrsanbindung und einige bekannte Ausflugsziele in Form von Gasthäusern herrscht ein hohes Interesse von Seiten der Erholungsnutzung vor. Die Gegend ist hügelig und es wechseln sich kleine Waldstücke mit Offenlandbereichen ab. Hochrangige Straßen kommen im Gegensatz zu den anderen Intensivuntersuchungsgebieten keine vor.

\section{Perchtoldsdorfer Heide}

Grundbesitzer und Auskunftspersonen: Gemeinde Perchtoldsdorf, ortskundige Mitarbeiter des Biosphärenparkmanagements.

Dieses Intensivuntersuchungsgebiet ist geprägt durch intensive Erholungsnutzung und das Vorhandensein der Indikatorarten Ziesel, Wildschwein und Rotwild. Es liegt wie das Intensivuntersuchungsgebiet Sievering nahe an Wien und erhält auch Zustrom aus den umliegenden Gemeinden. Besonders intensive Erholungsaktivitäten: Picknicken, Drachensteigen, Nachtaktivitäten, Aktivitäten mit Hunden (es gilt ein Leinenzwang auf der Perchtoldsdorfer Heide); darüber hinaus werden Krähen direkt und indirekt (durch Abfälle) gefüttert. Die Heide wird teils mit Schafen beweidet.

Die große Attraktion der Heide ist das Zieselvorkommen, das während der Vegetationsperiode wenn die Tiere aktiv sind viele Besucher anlockt. Dieses stellt durch sein dauerhaftes Bestehen trotz genetischer Isolation bei hohen Besucherzahlen ein interessantes Fallbeispiel dar. Unmittelbar im Bereich des Intensivuntersuchungsgebiets gibt es keine höherrangigen Straßen. Durch die Nähe zu Wien ist die Erreichbarkeit sehr gut gegeben. 


\subsubsection{Biosphärenpark Wienerwald und Umfeld}

Neben der Maßstabsebene der Intensivuntersuchungsgebiete werden Konfliktpotenziale im Projekt auf der Ebene des gesamten Biosphärenparks untersucht. Da die Biosphärenparkgrenzen sowohl für Besucherströme als auch für die betrachteten Tierarten keine praktische Wirkung besitzen, sollten im Projekt auch wesentliche funktionale Zusammenhänge mit der Umgebungsregion - soweit möglich und sinnvoll- berücksichtigt werden.

Das Umfeld des Biosphärenparks wirkt vor allem als Quell-Gebiet für die Erholungsnutzung. Der Biosphärenpark wird nicht nur von Ortsansässigen zur Erholung genutzt. Der Zustrom erfolgt primär aus Wien, aber auch aus den umliegenden größeren Städten wie St. Pölten oder Tulln.

Auch die Wildtierpopulationen stehen in Verbindung mit dem Umfeld des Biosphärenparks. Von den Indikator-Arten ist nur das Ziesel isoliert von anderen Beständen. Das Rotwild steht vor allem mit Beständen im Süden (Bezirk Wiener Neustadt und Neunkirchen) und im Westen (Bezirk Lilienfeld) in Verbindung. Schwarzwild kommt flächendeckend im Biosphärenpark und im Umfeld vor. Diese Tierart ist flexibler als das Rotwild, was die Nutzung von Korridoren und Wechseln anlangt. Dadurch ist das Schwarzwild trotz jagdlicher Regulierung im gesamten Biosphärenpark Wienerwald verbreitet. Die beiden Vogelarten Schwarzstorch und Auerhuhn stehen ebenfalls in Verbindung mit Vorkommen ausserhalb des Biosphärenparks. Die einzelnen Auerhuhnnachweise rekrutieren sich aus dem Vorkommensgebiet südlich des Biosphärenparks. Der Schwarzstorch brütet vereinzelt auch außerhalb der Parkgrenzen bzw. sucht sich seine Nahrung nicht nur im Park.

Verbindungen zwischen dem Biosphärenpark und dem Umfeld sind für Säugetiere im Süden und im Westen des Biosphärenparks ausreichend vorhanden. Richtung Norden und Osten dagegen beschränkt sich die großräumige Konnektivität auf einzelne Verbindungen. Diese Situation ergibt sich durch den Verlauf der Donau und die Lage Wiens in Relation zum Biosphärenpark Wienerwald. Beides stellt eine beinahe unüberwindbare Barriere, vor allem für große Säugetiere, dar. Damit ist auch die Verbindung über den Alpen - Karpaten - Korridor stark eingeschränkt. Die einzigen Verbindungsmöglichkeiten bestehen zwischen Baden und Bad Vöslau und südlich des Biosphärenparks bei Matzendorf. Weiters denkbar wäre noch eine Stelle bei Greifenstein an der Donau (pers.comm. Dr. Frey-Roos).

Die Lage des Biosphärenparks in der Großregion ist aus nachstehender Abbildung 24 ersichtlich. 


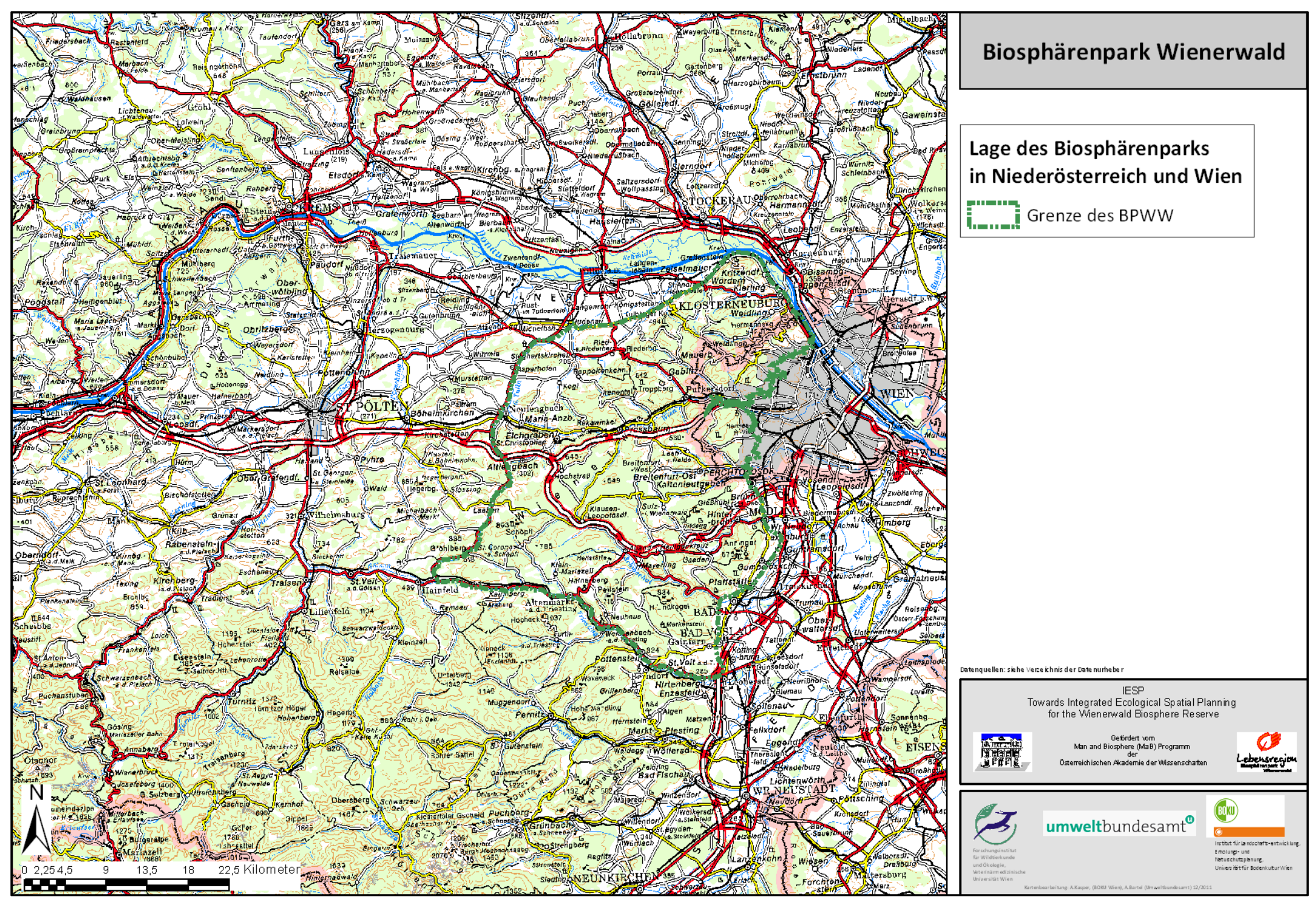

Abbildung 24: Lage des Biosphärenparks Wienerwald in der Großregion. 


\subsection{Stakeholderdialog}

Im Projekt wurde ein partizipativer Forschungsansatz angewendet, der sich unterschiedlicher Beteiligungsmethoden bediente, welche die drei wesentlichen Stufen der Beteiligungsintensität abdeckten: Information, Konsultation und Teilhabe an projektbezogenen Entscheidungen.

\subsubsection{Konsultative Methoden}

Konsultative Arbeitsmethoden bildeten einen starken Schwerpunkt der partizipativen Forschungsarbeit. In mehreren Projektphasen wurden Serien von Experteninterviews mit unterschiedlichen Zielsetzungen und Interviewpartnern durchgeführt. Die Experteninterviews folgten überwiegend dem Muster von strukturierten, leitfadengestützten face-to-face Tiefeninterviews; zum Teil wurden auch zusätzliche, geringer strukturierte Interviews zur Nachschärfung und Abrundung von Informationen durchgeführt.

Interviews wurden in unterschiedlichen Projektabschnitten und zu unterschiedlichen Zwecken zur Informationsgewinnung durchgeführt:

- Expertenbefragungen zu den Intensivuntersuchungsgebieten

- Expertenbefragungen zu den Indikator-Wildtierarten im Biosphärenpark

- Expertenbefragungen zu den Indikator-Freizeitaktivitäten im Biosphärenpark

- Expertenbefragungen zur Konfliktbehandlung auf der Perchtoldsdorfer Heide

Experteninterviews und -konsultationen werden ausführlicher in Kap. 2.3.1 beschrieben.

\subsubsection{Projektbegleitende Stakeholder-Arbeitsgruppe}

\subsubsection{Rahmen und Funktionen}

Als zentrales projektbezogenes Partizipationsorgan diente eine interdisziplinär und multisektoral zusammengesetzte Arbeitsgruppe von regionalen Stakeholdern. Durch diese wurden Vertreter relevanter regionaler Landnutzergruppen, Behörden, Betriebe und Grundeigentümer sowie Personen mit projektrelevanter Expertise im Biosphärenpark eng in das Projekt eingebunden. Die Beteiligungsintensität der Projektplattform lag zwischen „Konsultation“ und „Mitbestimmung" und kann daher am treffendsten als eine Form des Stakeholderdialogs charakterisiert werden.

Die Aufgaben der Arbeitsgruppe bestanden einerseits in der Information über und der kritischen Begleitung des Forschungsprojekts; darüber hinaus sollte die Mitwirkung unter anderem eine Bindung der Gruppenrepräsentanten an das Projekt hervorrufen, aber auch die Rückkopplung von Nutzergruppenangehörigen (Basis) mit ihren Vertretern in der Arbeitsgruppe - und damit mit dem Projekt - ermöglichen. Weiters sollte über die Arbeitsgruppe spezielles Regionalwissen über Tierarten, Freizeitaktivitäten, Landnutzungen und damit zusammenhängende Konfliktbereiche eingebracht und die Vorstellungen, Erwartungen und Visionen der verschiedenen Landnutzergruppen eingebracht werden. Letztlich sollten über die Teilhabe am Projekt und die Wirkungen des projektbezogenen Prozesses günstige Voraussetzungen geschaffen werden, um einen umsetzungsorientierten Folgeprozess nach Projektende zu initiieren. 
Von der Projektplattform ausgehend, wurden alle weiteren partizipativen Prozesse abgestimmt und gesteuert, z. B. die Auswahl von Interviewpartnern für Tiefeninterviews.

Die Arbeitsgruppe ist kein interessenpolitisches Abstimmungsgremium, sondern war als ein projektbegleitendes, konsultatives und kollaboratives Gremium im Rahmen eines partizipativen Forschungsansatzes konzipiert. Als solches war sie mit keinem politischen Mandat ausgestattet und besaß daher keine formale Entscheidungskompetenz. Ihre Aufgaben bestehen in erster Linie darin, regionalen Akteuren das Einbringen ihrer Standpunkte, von Erfahrungswissen und Zukunftsvorstellungen zu ermöglichen, laufende Arbeitsschritte kritisch zu begleiten, an der laufenden Schärfung und Nachjustierung von Forschungsfragestellungen mitzuwirken und erzielte Arbeitsergebnisse im Hinblick auf ihre praktische Umsetzbarkeit, Defizite und Verbesserungsmöglichkeiten zu diskutieren, wodurch letztlich auch die Akzeptanz von Projektergebnissen optimiert werden sollte.

\subsubsection{Zusammensetzung}

Ausgehend von einer Kerngruppe von Schlüssel-Stakeholdern wurde die Zusammensetzung der Arbeitsgruppe sukzessive erweitert. Die Kerngruppe bestand aus leitenden Vertretern des Biosphärenpark Wienerwald Managements, der größten Grundeigentümer im Biosphärenpark (Österreichische Bundesforste AG - Forstbetrieb Wienerwald; MA 49 - Forstamt und Landwirtschaftsbetrieb der Stadt Wien) und den politisch-administrativ zuständigen Biosphärenparkreferenten der Bundesländer Niederösterreich und Wien. In weiterer Folge wurden die gebietszuständigen Managementverantwortlichen (Forstrevierleiter, Jagdleiter, Grundeigentümervertreter) für die Intensivuntersuchungsgebiete, Artenschutzexperten und Naturschutzfachleute (Schwarzstorch, Ziesel), sowie jagdliche Interessenvertreter (Niederösterreichischer Landesjagdverband) sowie Vertreter unterschiedlicher Freizeitnutzergruppen (z. B. Ballonflugunternehmer, Laufclub, Mountainbikeverein, etc.) zugezogen. Bei der abschließenden Arbeitssitzung der Stakeholdergruppe wurde der Teilnehmerkreis schließlich auf politische Mandatarn und Verwaltungsverantwortlichen (Bezirkshauptleute, Landtagsabgeordnete, Bürgermeister) sowie VertreterInnen der Landesraumplanung ausgedehnt.

\subsubsection{Arbeitssitzungen und Themen}

Insgesamt fanden während der Projektlaufzeit vier offizielle Arbeitssitzungen der Dialogplattform statt, die jeweils unterschiedliche Rollen und Funktionen im Projekt erfüllten:

\section{Arbeitssitzung:}

- Projektvorstellung und Herstellung eines gemeinsamen Projektverständnisses

- Vorstellung und Abstimmung der Auswahlkriterien für Indikatorarten und -aktivitäten

- Diskussion der Auswahl von Indikatorarten und -aktivitäten

- Eingrenzung möglicher Intensivuntersuchungsgebiete

\section{Arbeitssitzung}

- Vorstellung von Kurzprofilen der ausgewählten Indikatorarten und -aktivitäten

- Diskussion der Auswahl und Beschlussfassung 
- Festlegung, Feinabgrenzung und erste Charakterisierung der Intensivuntersuchungsgebiete

- Identifizierung von gebietszuständigen InterviewpartnerInnen für die Intensivuntersuchungsgebiete

- Diskussion von Datenbedarf und Datenverfügbarkeit

\section{Arbeitssitzung}

- Vorstellung und Diskussion der Analyseergebnisse (Konfliktpotenziale) zu den Intensivuntersuchungsgebieten anhand von Karten:

o Objektivierung von Konfliktwahrnehmungen und „Eichung“ der Einschätzungen zwischen den Intensivuntersuchungsgebieten und TeilnehmerInnen

o Überprüfung von Befunden

o Verifikation bzw. Falsifikation von Arbeitshypothesen zu Konfliktmustern und Ursache-Wirkungs-Beziehungen

o Ergänzungen von zusätzlichen Informationen

- Integrative Diskussion von Gemeinsamkeiten und Unterschieden im Vergleich der Intensivuntersuchungsgebiete

- Schwerpunktsetzungen für die weitere Projektbearbeitung

\section{Arbeitssitzung}

- Vorstellung und Diskussion von Ergebnissen der biosphärenparkweiten GISBearbeitung:

o Methodik der GIS-Analyse

o Raumansprüche der Indikatorarten

o Räumliche Nutzungswahrscheinlichkeiten der Indikatoraktivitäten

o Konfliktpotenziale im Biosphärenpark

- Erste Diskussion von Handlungsstrategien für das Konfliktmanagement

\section{Arbeitssitzung}

- Vorstellung und Diskussion der Endergebnisse zu den biosphärenparkweiten Konfliktpotenzialen sowie Interpretationen und Schlussfolgerungen

- Vorstellung eines Diskussionsvorschlags zu Handlungsstrategien und Maßnahmenoptionen für das Konfliktmanagement; Bewertung und Diskussion in Arbeitsgruppen und im Plenum. 

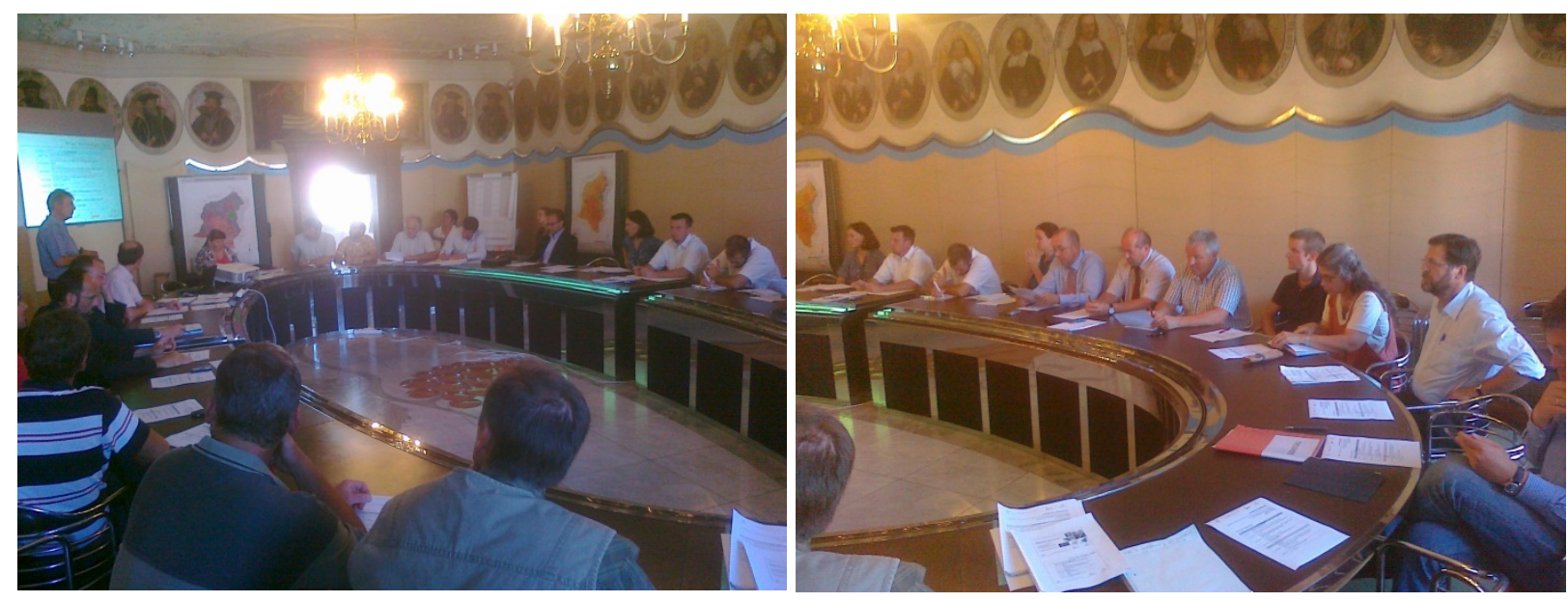

Abbildung 25: Fünftes Arbeitstreffen der Stake-

Abbildung 26: Fünftes Arbeitstreffen der Stakeholder-Arbeitsgruppe (Gemeinde Perchtoldsdorf) holder-Arbeitsgruppe (Gemeinde Perchtoldsdorf) 


\subsection{Konfliktanalyse}

\subsubsection{Ziel der Analyse}

Das vorliegende Projekt fokussiert auf eine Konfliktpotenzialanalyse. Die Analyse raumstruktureller Konfliktpotenziale zwischen dem Wildtiermanagement und der Freizeitnutzung bildet die Grundlage für differenzierte Strategien zur Konfliktvermeidung, -reduzierung und bewältigung im Biosphärenpark Wienerwald. Die Identifizierung und Bewertung von Konfliktpotenzialen kann zunächst unabhängig davon durchgeführt werden, ob bereits ein Konflikt ausgetragen wird oder nicht. Sie basiert auf einer Verknüpfung von Daten über die Lebensräume und das raum-zeitliche Verhalten der Wildtierarten mit Angaben zur Intensität der Freizeitnutzung und den raum-zeitlichen Nutzungsmustern der Freizeitaktivitäten. Die daraus abgeleiteten Konfliktpotenziale werden nicht nur verortet (potenzielle Konflikträume), sondern auch in ihrem Ursachen- und Wirkungsgefüge beschrieben. Aus ihren unterschiedlichen Ausprägungen sollen Maßnahmen für ein Wildtiermanagement vorgeschlagen werden, das mögliche Konflikte bereits im Vorfeld berücksichtigt und eine Konfliktvermeidung anstrebt.

Die Analyse bereits ausgetragener oder zumindest latent bestehender Konflikte dient ergänzend dazu der Erfassung von Konfliktstrukturen und Konfliktmanagementstrategien. Ausgangspunkt sind die verschiedenen Konfliktparteien, ihre Interessen, Ziele, Nutzungsansprüche und Nutzungskonkurrenzen sowie die wechselseitigen Aktionen und Reaktionen im Konfliktprozess. Eine Konfliktanalyse wird im Rahmen des Projektes nur exemplarisch durchgeführt, um Ansatzpunkte für ein Konfliktmanagement zu ermitteln, das nicht nur die (wild-) ökologische Sicht, sondern auch die Nutzersicht einbezieht.

\subsubsection{Analyseraster für Konfliktpotenziale und Konflikte im Wienerwald}

Das Analyseraster für die Erfassung und Darstellung der Konfliktpotenziale und Konflikte im Biosphärenpark Wienerwald ist aus den theoretisch-methodischen Arbeiten zu Konfliktanalyse und -management von Berkel, Glasl und Ziener (vgl. Kapitel 2.2.4.1) abgeleitet. Im Folgenden wird zunächst das grundlegende Analyseraster für die Konfliktpotenzial- und Konfliktanalyse erläutert. Dieses wird dann anschließend für die beiden Untersuchungsebenen Intensivuntersuchungsgebiete und gesamter Biosphärenpark - modifiziert, um den unterschiedlichen Zielstellungen und Datengrundlagen Rechnung zu tragen.

\section{A. Konfliktpotenzial}

1. Charakteristik des Konfliktpotenzials zwischen Indikatorart und Indikatoraktivität

Um das jeweilige Konfliktpotenzial verbal zu beschreiben, werden zunächst das Vorkommen der Wildtierart und die ausgeübte Freizeitaktivität kurz charakterisiert.

2. Potenzielle Konflikträume

Die räumliche Verortung der Konfliktpotenziale erfolgt durch eine Überlagerung der raumbezogenen Daten zu den Wildtierarten und den Freizeitaktivitäten in einer Karte. Die auf diese Weise ermittelten potenziellen Konflikträume können allerdings nicht flächenscharf abgegrenzt, sondern lediglich gekennzeichnet werden. Eine genauere Abgrenzung potenzieller Konflikträume würde weder der Dynamik der Wildtierlebensräume und der aktivitätsspezifischen Nutzungsmuster der Freizeitnutzergruppen entsprechen 
noch könnte sie der Datensituation und Bewertungsmethodik Rechnung tragen (vgl. Kap. 2.6.4).

\section{Zeitliche Aspekte}

Die zeitlichen Aspekte der Konfliktpotenziale resultieren zum einen aus den tages- und jahreszeitlichen Rhythmen und Sensibilitätsunterschieden der Wildtierarten und zum anderen aus einem unterschiedlichen Aktionsradius und differenzierten Nutzungsmustern bei den Freizeitaktivitäten zu den verschiedenen Tages- und Jahreszeiten sowie innerhalb der Woche und Wochenende.

\section{Stärke des Konfliktpotenzials}

Die Bestimmung der Konfliktstärke bedeutet zugleich, dass die Schwelle, ab der man überhaupt von einem Konfliktpotenzial ausgehen kann, festgelegt wird. Nachdem sowohl bei den Wildtierarten als auch bei den Freizeitaktivitäten geringe Vorkommen bzw. Nutzungsintensitäten zumeist nicht eindeutig vom Nichtvorhandensein unterschieden werden können, stellt gerade dies ein Problem dar. Für die Stärke des Konfliktpotenzials wird daher folgende dreistufige Skala festgelegt:

- kein oder geringes Konfliktpotenzial,

- mittleres Konfliktpotenzial,

- hohes Konfliktpotenzial.

Ermittelt wird die Konfliktstärke aus einer ebenfalls jeweils dreistufigen Skala der Wildtiervorkommen (z.B. hohe, mittlere, geringe Nachweisdichte beim Schwarzstorch) und der Freizeitaktivitäten (z.B. hohe, mittlere, geringe Intensität) mittels einer Matrix.

\section{Ursachen und Wirkungsgefüge}

Die inhaltliche Beschreibung des Konfliktpotenzials und seiner räumlichen und zeitlichen Ausprägung schließt die Darstellung von Ursachen und Wirkungszusammenhängen ein. Wechselwirkungen entstehen vor allem durch die unterschiedliche Sensibilität der Wildtierarten gegenüber anthropogenen Störungen und die unterschiedliche Störwirkung der einzelnen Freizeitaktivitäten.

6. Auswirkungen auf andere Bereiche

Bereits die Konfliktpotenziale verdeutlichen unterschiedliche Wechselwirkungen mit Bereichen wie Jagd, Landwirtschaft, Weinbau, Siedlungsentwicklung und vor allem dem Biosphärenparkmanagement, insbesondere in den Kernzonen.

\section{B. Konflikt}

1. Konfliktparteien

Die beteiligten Konfliktparteien sind zu erfassen und ihre Interessen, Ziele und Nutzungsansprüche zu charakterisieren. Dabei sind nicht nur konträre Interessen, Ansprüche und Positionen zu berücksichtigen, sondern auch gemeinsame Interessen, die Kooperationen und Partnerschaften ermöglichen.

\section{Konfliktgegenstand}

Der objektive Konfliktgegenstand ergibt sich aus dem Konfliktpotenzial. Seine Beschreibung ist erforderlich, um eine Verbindung zwischen dem analysierten Konfliktpotenzial und dem Konfliktergebnis herstellen zu können. Nur dadurch lassen sich die (langfristi- 
gen) Wirkungen der Konfliktaustragung und -bewältigung auf die Wildtierlebensräume und die Freizeitnutzung beurteilen.

3. Streitpunkte

Die von den Konfliktbeteiligten formulierten Streitpunkte stellen die subjektiven Auffassungen der Konfliktparteien vom Konfliktgegenstand dar. Sie bilden den Ansatzpunkt für das Konfliktmanagement (z.B. Worum geht es den Konfliktparteien? Schätzen sie die Situation ähnlich ein? Wie erleben Sie die Streitpunkte?)

4. Konfliktform und Konfliktverlauf

Neben der Einordnung als Sach- oder Verteilungskonflikt kann auch ein Raumnutzungskonflikt Aspekte eines Beziehungskonflikts beinhalten, die dann eine andere Konfliktbewältigungsstrategie erfordern. Zur Konfliktform gehören insbesondere die Mittel, mit denen der Konflikt ausgetragen wird (z.B. durch persönliche Auseinandersetzungen, über die Medien, institutionalisiert) und in welcher Schärfe dies erfolgt (z.B. Beschimpfungen, Bedrohungen). Der Konfliktverlauf kann durch Phasen beschrieben und in seiner Entwicklungsrichtung charakterisiert werden (verstärkt, abgeschwächt). Eine tiefer gehende Analyse erfasst die Aktivitäten und Positionen der Konfliktparteien und ihre wechselseitigen Reaktionen bei der Konfliktaustragung und -bewältigung.

5. Konfliktergebnis

Sofern bereits ein Konfliktergebnis vorliegt ist dieses festzuhalten und im Hinblick auf das Konfliktpotenzial zu bewerten.

\subsubsection{Modifizierung der Analyseschritte auf den Untersuchungsebenen}

\subsubsection{Intensivuntersuchungsgebiete}

In den vier Intensivuntersuchungsgebieten basiert die Konfliktpotenzialanalyse im Wesentlichen auf den Ergebnissen leitfadengestützter Interviews mit regionalen Experten (vgl. Kapitel 2.3.1). In den Interviews wurden die Vorkommen der Indikatorarten und die Nutzungsintensität der Indikator-Freizeitaktivitäten erfasst und in Arbeitskarten dargestellt. Diese bilden die Grundlage für die Bestimmung der Hauptkonfliktpotenziale und potenziellen (Haupt-) Konflikträume.

Als Hauptkonfliktpotenziale werden diejenigen Konfliktpotenziale angesehen, die im jeweiligen Intensivuntersuchungsgebiet von Bedeutung sind. Ihre Bestimmung erfolgte aufgrund der Experteninterviews und der kartographischen Darstellung der Wildtiervorkommen und der Nutzungsmuster der Freizeitaktivitäten. Die weitere Analyse konzentriert auf diese Hauptkonfliktpotenziale, so dass nicht alle Kombinationen zwischen den vorkommenden Wildtierarten und den ausgeübten Freizeitaktivitäten kartographisch dargestellt und analysiert werden. Die potenziellen Konflikträume entstehen durch die kartographische Überlagerung der Wildtiervorkommen mit den Nutzungsmustern und -intensitäten der Freizeitaktivitäten (vgl. Kap. 2.6.4).

In die textliche Beschreibung der Konfliktpotenziale und die Erläuterung der Ursachen und Wirkungszusammenhänge sowie Wechselbeziehungen zu anderen Bereichen fließen die umfangreichen Informationen der Interviewpartner sowie weitere Recherchen ein. So wurden beispielsweise raum-zeitliche Differenzierungen bei den Freizeitaktivitäten (z. B. Freizeitaktivitäten innerhalb der Woche und am Wochenende, jahreszeitliche Unterschiede) und tages- 
und jahreszeitlich unterschiedliche Sensibilität der Wildtierarten gegenüber Störungen einbezogen. Die Charakterisierung des Ursachen-Wirkungsgefüges resultiert aus dem Beziehungsgefüge der jeweiligen Wildtierarten und Freizeitaktivitäten.

Die Stärke des Konfliktpotenzials wird für die einzelnen Konfliktpotenziale zwischen Wildtierart und Freizeitaktivität nicht bestimmt. Stattdessen werden die potenziellen Hauptkonflikträume in den einzelnen Intensivuntersuchungsgebieten durch eine Überlagerung der Hauptkonfliktpotenziale dargestellt (vgl. Kap. 2.6.4).

Eine Konfliktanalyse wird nur auf der Ebene der Intensivuntersuchungsgebiete durchgeführt. Diese basiert auf den Einschätzungen der regionalen Experten. In den Interviews zu den Intensivuntersuchungsgebieten wurde auch erhoben, inwieweit die Probleme (bzw. Konfliktpotenziale) bereits zur Austragung von Konflikten geführt haben. Wenn dies der Fall war, wurden ausgewählte Merkmale der Konflikte erfragt, insbesondere die Konfliktparteien, der Konfliktlösungsprozess und Konfliktergebnisse. Dies erlaubt eine grobe Beschreibung der aufgetretenen Konflikte und ihrer Bewältigung. Auf Details, etwa zu den Streitpunkten und dem Konfliktverlauf, kann und soll in diesem Zusammenhang nicht eingegangen werden.

\subsubsection{Biosphärenpark Wienerwald}

Auf der Ebene des Biosphärenparks wurden die Ausgangsdaten für die Konfliktpotenzialanalyse durch unterschiedliche Methoden erfasst und aufbereitet. Die Vorkommen der einzelnen Wildtierarten wurden wiederum weitgehend in Experteninterviews ermittelt, in denen auch eine kartographische Darstellung erfolgte. Ergänzend hierzu wurden Abschussdaten der jagdbaren Arten sowie vorliegende Studien, wie Frank und Berg 2001, einbezogen. Die entstehende Vorkommensverteilung bei den einzelnen Wildtierarten wird als Vorkommenswahrscheinlichkeit interpretiert (vgl. Kap. 3.4.2).

Bei den Freizeitaktivitäten bilden aktivitätsspezifische Quellgebiets- und Zielgebietsparameter die Grundlage für eine GIS basierte Ermittlung von Nutzungswahrscheinlichkeiten. Diese basieren auf den räumlichen und zeitlichen Anforderungen und Nutzungsmotiven sowie soziodemographischen Nutzerprofilen für die einzelnen Freizeitaktivitäten, die aufgrund von Literaturrecherchen, Ergebnissen des Vorgängerprojekts ISWI-MAB (vgl. Reimoser et al. 2008) und ergänzenden Experteninterviews erstellt worden sind (vgl. Kap. 2.3.1.3 und Kap. 3.2.2).

Den Schwerpunkt der Konfliktpotenzialbestimmung auf der Ebene des Biosphärenparks bildet die kartographische Überlagerung der Vorkommenswahrscheinlichkeiten der einzelnen Wildtierarten und der Nutzungswahrscheinlichkeiten der einzelnen Freizeitaktivitäten. (vgl. Kap. 2.6.4 und Kap. 3.4.3). Daraus resultieren ebenfalls räumlich differenzierte Konfliktpotenziale als Kombinationen zwischen vier Indikator-Wildtierarten und fünf IndikatorFreizeitaktivitäten. Das Ziesel wird nicht betrachtet, weil das Vorkommen im Wienerwald auf die Perchtoldsdorfer Heide beschränkt ist und das Picknicken/Grillen kann nicht einbezogen werden, weil die entsprechenden Daten auf Biosphärenparkebene fehlen.

Die verbale Beschreibung der Konfliktpotenziale beinhaltet im Wesentlichen die räumliche Verteilung der einzelnen Konfliktpotenziale in ihrer relativen Größe. Die Konfliktpotenziale bilden somit nur die Spanne zwischen geringem und hohem Konfliktpotenzial in ihrer räumlichen Ausprägung ab. Eine absolute Stärke des Konfliktpotenzials kann aus dieser Modellierung nicht ermittelt werden. Die Charakterisierung der Konfliktpotenziale bezieht sich auf die überlagerten Vorkommenswahrscheinlichkeiten der Wildtierart und der Nutzungswahrscheinlichkeiten der Freizeitaktivität. Eine weiterreichende Erläuterung der Konfliktpotenziale hinsichtlich zeitlicher Aspekte oder der Wirkungszusammenhänge erfolgt auf dieser Ebene nicht. Die räumliche Verteilung der Konfliktpotenziale gibt darüber hinaus auch Auskunft über 
potenzielle Konflikträume. Aufgrund der verwendeten Modellparameter und der Überlagerung von Vorkommens- und Nutzungswahrscheinlichkeiten lassen sich diese aber nur großräumig differenzieren.

\subsubsection{Kartographische Überlagerung der Daten und Bewertungsergebnisse}

Den zentralen Teil der Konfliktpotenzialanalyse bilden die kartographische Darstellung der Konfliktpotenziale und die Bestimmung potenzieller Konflikträume. Dies erfolgt durch eine Überlagerung der Wildtierlebensräume mit den räumlichen Nutzungsmustern der Freizeitaktivitäten. Entsprechend der unterschiedlichen Datenlage unterscheidet sich dabei wiederum die Methodik auf den beiden Untersuchungsebenen.

\subsubsection{Kartographische Überlagerung der Wildtierlebensräume und der Intensität der Freizeitaktivitäten in den Intensivuntersuchungsgebieten}

In den Intensivuntersuchungsgebieten basiert die räumliche Darstellung der Konfliktpotenziale auf den in den Experteninterviews erstellten Arbeitskarten (Grundlage war die ÖK 50.000, vergrößert auf das jeweilige Intensivuntersuchungsgebiet) sowie deren Digitalisierung. Sowohl die Lebensräume der einzelnen Wildtierarten als auch die Nutzungsintensität der verschiedenen Freizeitaktivitäten und der Freizeitnutzung insgesamt werden hierbei in einer dreistufigen Skala dargestellt. Im IG Sievering wurden im Interview die deutlich kleinteiligeren Nutzungsmuster von Wildtieren und Freizeitnutzern in einem größeren Maßstab betrachtet und nachträglich generalisiert.

Bei den Vorkommen der Wildtiere wird prinzipiell zwischen Hauptlebensräumen (z.B. Kerngebiete des Rotwildes, aktuelle Auerwildnachweise), sonstigen Lebensräumen und Randgebiete unterschieden. Diese idealtypische Gliederung der Lebensräume ist aber nur beim Rotwild erkennbar. Sie wird ergänzt durch eine Reihe weiterer raumbezogener Informationen zu den Rotwildlebensräumen wie Ruhezonen, Fütterungsstellen, Wildwechsel und Barrieren. Die Schwarzwildlebensräume werden nur im IG Sievering und im IG Perchtoldsdorfer Heide näher untersucht und insbesondere durch Einstände und Wildwechsel beschrieben. Die Darstellung der Schwarzstorch- und Auerwildlebensräume basiert im Wesentlichen auf Sichtungen. Zusätzlich werden für beide Wildtierarten Potenzialgebiete auf der Basis ausgewählter Lebensraumeigenschaften dargestellt. Beim Ziesel gibt es nur auf der Perchtoldsdorfer Heide ein Vorkommen, welches in ein starkes und ein geringes Vorkommen gegliedert und mit einem Potenzialgebiet dargestellt wird.

Für die Freizeitnutzung insgesamt wurden in den Expertengesprächen die Kategorien „intensive Nutzung“, „Nutzung vorhanden“ und „kaum bzw. gar keine Nutzung“ erfasst. Dies bedeutet einerseits eine flächenhafte Darstellung von Gebieten mit intensiver Freizeitnutzung und Gebieten, die kaum oder gar nicht für Freizeitzwecke genutzt werden, und andererseits eine Kennzeichnung der stark bzw. sehr stark genutzten Wege. Bei den einzelnen Freizeitaktivitäten konnte diese Abstufung nicht immer umgesetzt werden. Die Nutzungsintensität beim Mountainbiking wurde nur im IG Lindkogel mit einem flächigen Intensivgebiet beschrieben. Dies resultiert aus der Wahrnehmung, dass beim Mountainbiking die Wege häufig verlassen bzw. immer wieder neue Pfade angelegt werden, so dass ein flächenhafter Eindruck entsteht. Ansonsten wurde wie bei den beiden anderen linienhaften Freizeitaktivitäten Joggen und Aktivitäten mit dem Hund zwischen einer starken und schwachen (vorhandenen) Nutzung von Wegen unterschieden. Zu berücksichtigen ist dabei allerdings, dass stark genutzte Wege in den IGs Hirschenstein und Hoher Lindkogel quantitativ anders zu beurteilen sind als stark genutzte Wege in den IGs Perchtoldsdorfer Heide und Sievering (vgl. Kap. 
3.3). Beim Picknicken wurden die stark genutzten Picknick- bzw. Grillplätze markiert, sowohl offizielle als auch illegal genutzte Flächen. Eine Differenzierung der Nutzungsintensität erfolgte hierbei nicht. Geocaching ist die einzige Freizeitaktivität, deren Nutzungsmuster in den Intensivuntersuchungsgebieten nicht durch die Expertengespräche, sondern nach den vorhandenen Geocaches ermittelt wurde. Das Geocaching war zum Zeitpunkt der Interviews noch zu wenig bekannt, so dass noch keine Erfahrungen vorlagen und die Bestimmung von Nutzungsintensitäten nicht möglich war. Beim Ballonfahren war es schließlich nur möglich, die im Intensivuntersuchungsgebiet oder dem unmittelbaren Umfeld gelegenen Startplätze, die Hauptflugrichtungen und bekannte Landesplätze zu kennzeichnen.

Durch die Überlagerung der Wildtierlebensräume mit der räumlich differenzierten Nutzungsintensität der Freizeitaktivitäten und der gesamten Freizeitnutzung werden die potenziellen Konflikträume sichtbar. Dabei ist zu berücksichtigen, dass die Intensitätsabstufungen weder bei den Wildtierlebensräumen noch bei den Freizeitaktivitäten durch eine Linie abgrenzbar sind. Zwischen ihnen sind eher Grenzbereiche anzunehmen. Demzufolge erfolgt auf der Ebene der wildtier- und aktivitätsspezifischen Konfliktpotenziale keine Verschneidung der Flächen. Die mit den Konfliktpotenzialen verbundenen potenziellen Konflikträume werden durch die Überlagerung der verschiedenen Signatur sichtbar und im Text beschrieben.

Die Überlagerung der Wildtiervorkommen und Nutzungsmuster liefert die potenziellen Hauptkonflikträume für jedes Intensivuntersuchungsgebiet. Grundlage ist wiederum eine dreistufige Skala: potenzielle Hauptkonflikträume, weitere potenzielle Konflikträume, kein potenzieller Konfliktraum. Die vorgenommene Differenzierung ergibt sich aus der Überlagerung der einzelnen Wildtiervorkommen mit den verschiedenen Freizeitaktivitäten. In den potenziellen Hauptkonflikträumen führt die Beteiligung von mehreren Wildtierarten und/oder Freizeitaktivitäten zur Entstehung mehrerer Konfliktpotenziale. In den anderen potenziellen Konflikträumen liegt jeweils ein Konfliktpotenzial vor. Eine Aussage zur Stärke der Konfliktpotenziale oder der zu erwartenden Konflikte ist daraus nicht ableitbar. Ein Vergleich zwischen den vier Intensivuntersuchungsgebieten ist ebenfalls nicht möglich.

\subsubsection{Kartographische Überlagerung der Vorkommenswahrscheinlichkeit der Wild- tierarten und der Nutzungswahrscheinlichkeiten der Freizeitaktivitäten im Bio- sphärenpark und dessen Umfeld}

Auf der Ebene des Biosphärenparks und seines Umfelds erfolgte die Erfassung und kartographische Darstellung der Wildtiervorkommen in ähnlicher Weise wie in den Intensivuntersuchungsgebieten. Die grundlegenden Informationen wurden wiederum durch Experteninterviews gewonnen, die durch einzelne weitere Daten ergänzt wurden (siehe oben). Dabei wird die Erfassung der Wildtierlebensräume nicht auf den Biosphärenpark begrenzt, sondern dort, wo es Beziehungen zum Umfeld gibt, diese mit einbezogen. Das betrifft insbesondere großräumige Beziehungen (Korridore) oder Barrieren.

Die dreistufige Differenzierung der Wildtierlebensräume wird auf der Ebene des gesamten Biosphärenparks beibehalten. Für die einzelnen Wildtierarten sind jedoch Modifizierungen notwendig. Beim Rotwild wird zwischen Kerngebiet, Randgebiet und rotwildfreiem Gebiet unterschieden. Zusätzlich werden Wintereinstandsgebiete dargestellt. Da das Schwarzwild im gesamten Wienerwald vorkommt, gibt es nur die Kategorien Hauptlebensraum und Vorkommen. Für beide Tierarten werden außerdem wichtige Wildwechsel erfasst. Für den Schwarzstorch und das Auerwild erfolgt die Darstellung der Lebensräume auf der Grundlage von Nachweisen. Aus ökologischen und rechtlichen Gründen war es notwendig, die punktförmigen Schwarzstorchnachweise nach ihrer Dichte in Flächen zusammenzufassen. Die Vorkommenswahrscheinlichkeit beim Schwarzstorch gliedert sich somit in Areale mit hoher, mittlerer und geringer Schwarzstorch-Nachweisdichte. Aufgrund der wenigen aktuellen Auerwild- 
Sichtungen im Gebiet des Biosphärenparks Wienerwald werden für diese Wildtierart auch historische Vorkommen einbezogen. Ein Auerwildkerngebiet befindet sich jedoch südlich des Triestingtals im unmittelbaren Umfeld des Biosphärenparks. Mit Blick auf eine mögliche Wiederausbreitung von Auerwild sind darüber hinaus potenzielle Lebensräume gekennzeichnet. Das Ziesel wird auf Grund des einzigen Vorkommens auf der Perchtoldsdorfer Heide (Intensivuntersuchungsgebiet) auf der Ebene des gesamten Biosphärenparks nicht betrachtet (zur detaillierten Methodik vgl. Kap. 3.2.1).

Die räumlich differenzierten Nutzungswahrscheinlichkeiten der verschiedenen Freizeitaktivitäten werden auf der Ebene des Biosphärenparks durch eine GIS basierte Abschätzung eines aktivitätsspezifischen Sets von Indikatoren ermittelt, die sich zum einen auf die Quellgebiete der Freizeitnutzer und zum anderen auf Eigenschaften der Zielgebiete beziehen. Die Ergebnisse dieser Modellierung sind somit lediglich als Nutzungswahrscheinlichkeiten zu interpretieren, sie kennzeichnen aber nicht die aktuellen Nutzungsmuster der Freizeitaktivitäten. Als Quellgebiete werden neben dem Biosphärenpark auch die Siedlungen im unmittelbaren Umfeld einbezogen. Als Zielgebiet kann auf Grund der Datenlage dagegen nur der Biosphärenpark Wienerwald selbst berücksichtigt werden, so dass die Nutzungswahrscheinlichkeiten ebenfalls nur für das Gebiet des Biosphärenpark vorliegen. Grundlage für die Quell- und Zielgebietsparameter sind Befragungen im Wienerwald (Projekt ISWI-MAB, vgl. Reimoser et al. 2008), Literaturrecherchen und einige zusätzliche Experteninterviews, um die Informationen zu den einzelnen Indikator-Freizeitaktivitäten zu komplettieren. Die Nutzungswahrscheinlichkeit der Aktivität Picknicken kann aufgrund unzureichender Daten nicht für den gesamten Biosphärenpark ermittelt werden, so dass hier auch kein Konfliktpotenzial mit den Wildtierarten dargestellt werden kann. Zur Darstellung der räumlich differenzierten Nutzungswahrscheinlichkeit der einzelnen Freizeitaktivitäten wird zunächst eine fließende Einteilung verwendet, um statt scharfer Grenzen, die schwer interpretierbar sind, Übergangsbereiche abzubilden (zur detaillierten Methodik vgl. Abschnitt 3.2.2).

Die Konfliktpotenziale werden durch eine kartographische Überlagerung der Vorkommenswahrscheinlichkeiten der einzelnen Wildtierarten mit den Nutzungswahrscheinlichkeiten der verschiedenen Freizeitaktivitäten ermittelt. Im Ergebnis dessen entsteht eine Konfliktpotenzialkarte, die die relativen Konfliktpotenziale im gesamten Biosphärenpark Wienerwald abbildet. Relativ bedeutet, dass für jede Kombination zwischen Wildtierart und Freizeitaktivität jeweils das höchste und geringste Konfliktpotenzial ermittelt wird und zwischen diesen die Werte in einer kontinuierlichen Abstufung dargestellt werden. Die Konfliktpotenzialkarten verdeutlichen somit die räumliche Differenzierung der Konfliktpotenziale innerhalb des Wienerwalds. In einem zweiten Schritt werden für jede Indikator-Wildtierart die Konfliktpotenziale mit allen Indikator-Aktivitäten zusammengefasst. Die entsprechenden Karten veranschaulichen somit das Konfliktpotenzial zwischen jeweils einer Wildtierart und der Freizeitnutzung, repräsentiert durch die Indikator-Freizeitaktivitäten bzw. deren summarische Zusammenfassung.

Für eine Zusammenschau der Konfliktpotenziale im Wienerwald erfolgt in einem weiteren Schritt die Verknüpfung aller Einzel-Konfliktpotenziale zwischen den Wildtierarten und den Freizeitaktivitäten, wobei unterschiedliche Verfahren verwendet werden. Beim ersten Verfahren werden an jedem Punkt alle Potenzialwerte summiert und in einer Gesamtkonfliktpotenzial-Karte die relativierten Werte in einer kontinuierlichen Abstufung von einem geringen bis zu einem hohen Konfliktpotenzial dargestellt. Beim zweiten Verfahren wird jeweils das Konfliktpotenzial des höchsten Einzelpotenziales berücksichtigt und wiederum das relativierte Gesamtkonfliktpotenzial bestimmt. Auf der Basis dieser Maximalwerte wird außerdem qualitativ veranschaulicht, welches das jeweils dominierende Konfliktpotenzial zwischen Wildtierart und Freizeitaktivität darstellt (vgl. Kap. 3.4.3.5). 


\subsubsection{Konflikttypisierung}

Typisierungen oder Typologien werden immer dann angestrebt, wenn Objekte oder Sachverhalte einerseits sehr vielfältig sind, andererseits aber Gemeinsamkeiten oder Ähnlichkeiten aufweisen. Durch die Beschreibung der "typischen" Merkmale von Objektgruppen werden zugleich Grundlagen für weitere Analysen, Konzepte oder Handlungsschritte geschaffen. Es gibt eine ganze Reihe von Konflikttypisierungen, darunter Verteilungskonflikte, Sachund Beziehungskonflikte, heiße und kalte Konflikte. Die im vorliegenden Projekt vorgenommene Typisierung von Konfliktpotenzialen und Konflikten basiert auf einer Typisierung von Raumnutzungs- und Zielkonflikten zwischen Erholungsnutzung und Naturschutz. Diese erfolgte in einem deduktiv-induktiven Verfahren auf der Grundlage der Strukturelemente eines Konflikts und einer Analyse von Konflikten in sechs Regionen in Deutschland und Österreich (vgl. Ziener, 2003). Von den dabei beschriebenen acht Typen von Raumnutzungskonflikten können zwei für die Analyse von Konflikten zwischen Wildtierökologie und Freizeitnutzung adaptiert werden. Die Analyse von raumstrukturellen Konfliktpotenzialen und Konflikten in den Intensivuntersuchungsgebieten im Wienerwald hat bestätigt, dass sich bei Konflikten zwischen Wildtierökologie und Freizeitnutzung zwei grundlegende Konflikttypen unterscheiden lassen (vgl. Abbildung 27):

- Freizeitaktivitäten in wildökologisch sensiblen Räumen und

- Mangelnde Akzeptanz und Verstöße von Einzelnen gegen Nutzungsrestriktionen.

Die beiden Konflikttypen fokussieren auf unterschiedliche Aspekte der Konfliktpotenziale und Konfliktaustragung. Die Lösungsmöglichkeiten und Konfliktmanagementstrategien sind dementsprechend unterschiedlich. Der erste Konflikttyp beinhaltet die grundsätzliche Ausübung von Freizeitaktivitäten in sensiblen Räumen, einschließlich bestimmter Trends wie die Ausdehnung der Aktivitäten in die Abend- und Nachtstunden mit Hilfe von Stirnlampen. Das raumstrukturelle Konfliktpotenzial besteht in dem Gegensatz zwischen der Sensibilität von Wildtieren gegenüber anthropogenen Einflüssen und der Störwirkung von Freizeitaktivitäten auf die Wildtiere bzw. ihre Lebensräume (ökologische Sichtweise). Die Lösungsansätze in diesem Konflikttyp sind eher struktureller Art, beispielsweise Maßnahmen zur Funktionstrennung (z. B. Zonierung) und Besucherlenkung. Sie setzt somit bei den raumstrukturellen Konfliktpotenzialen an und kann bereits auf dieser Ebene erfolgen noch bevor ein Konflikt ausgetragen wird. Entscheidend für die Umsetzung entsprechender Maßnahmen und Konzepte und eine Konfliktprävention ist die frühzeitige Einbindung aller Interessengruppen, die eine gemeinsame Entwicklung von Maßnahmen oder Nutzungsrestriktionen ermöglicht. Dadurch können zwar Konflikte um Freizeitaktivitäten in wildbiologisch sensiblen Räumen nicht endgültig gelöst, aber die Konfliktpotenziale zumindest partiell reduziert bzw. entschärft werden. 


\begin{tabular}{|c|c|c|}
\hline & $\begin{array}{c}\text { Freizeitaktivitäten in wildökologisch } \\
\text { sensiblen Räumen }\end{array}$ & $\begin{array}{c}\text { Verstöße Einzelner gegen Nutzungs- } \\
\text { restriktionen }\end{array}$ \\
\hline $\begin{array}{l}\text { Konflikt- } \\
\text { potenzial }\end{array}$ & $\begin{array}{c}\text { Wildtierökologische Sicht: } \\
\text { Sensibilität der Wildtierarten gegenüber } \\
\text { anthropogenen Störeinflüssen } \\
><\end{array}$ & $\begin{array}{c}\text { Nutzersicht: } \\
\text { Nutzungsrestriktionen des Naturschutzes / } \\
\text { BPs, Wildtiermanagements ... } \\
>< \\
\text { Nutzungsansprüche und -verhalten } \\
\text { der Freizeitnutzergruppen }\end{array}$ \\
\hline $\begin{array}{l}\text { Konflikt- } \\
\text { raum }\end{array}$ & $\begin{array}{c}\text { Überlagerung der Wildtierlebensräume } \\
\text { und der Nutzungsintensitäten bzw. } \\
\text { Nutzungswahrscheinlichkeiten } \\
\text { (Potenzialcharakter) }\end{array}$ & $\begin{array}{c}\text { eher kleinräumig, linienhaft (Wege), } \\
\text { punktuell } \\
\text { (nur durch Beobachtung oder Reflektion } \\
\text { im Interview erfassbar) }\end{array}$ \\
\hline $\begin{array}{l}\text { Konflikt- } \\
\text { austra- } \\
\text { gung }\end{array}$ & $\begin{array}{l}\text { zw. Organisationen und Institutionen, } \\
\text { z.B Naturschutz, Tourismus, Freizeitsport, } \\
\text { Forstverwaltung, BP, Gemeinde, ... }\end{array}$ & $\begin{array}{c}\text { mit Einzelpersonen / Gruppen, z.B. } \\
\text { Mountainbikern, Personen mit Hund, } \\
\text { (offene Auseinandersetzungen - verbal, } \\
\text { teilweise medial) }\end{array}$ \\
\hline $\begin{array}{l}\text { Konflikt- } \\
\text { lösungs- } \\
\text { ansätze }\end{array}$ & $\begin{array}{c}\text { Funktionstrennung, } \\
\text { Nutzungsrestriktionen, } \\
\text { Besucherlenkung, Leitsysteme }\end{array}$ & $\begin{array}{c}\text { zielgruppenorientierte Information, } \\
\text { Präsenz, Kontrollen, } \\
\text { Sanktionen }\end{array}$ \\
\hline $\begin{array}{l}\text { Konflikt- } \\
\text { lösungs- } \\
\text { prozess }\end{array}$ & $\begin{array}{l}\text { Verhandlungen, Regelungen, } \\
\text { gemeinsame Erarbeitung von Konzepten, } \\
\text { Kompromisse }\end{array}$ & $\begin{array}{c}\text { Doppelstrategie aus zielgruppenorientier- } \\
\text { ter Information und Aufklärung } \\
\text { und massiven Sanktionen gegenüber } \\
\text { einzelnen Uneinsichtigen }\end{array}$ \\
\hline $\begin{array}{l}\text { Konflikt- } \\
\text { ergebnis }\end{array}$ & $\begin{array}{l}\text { Konflikt prinzipiell dauerhaft, nicht endgül- } \\
\text { tig lösbar, häufig Wechsel von latenten } \\
\text { und offen ausgetragenen Konflikten }\end{array}$ & $\begin{array}{c}\text { in der Gesamtheit dauerhaft, } \\
\text { mit den Einzelpersonen zeitweilig, aber } \\
\text { u.U. länger andauernd }\end{array}$ \\
\hline $\begin{array}{l}\text { Konflikt- } \\
\text { wirkungen }\end{array}$ & $\begin{array}{l}\text { Teillösungen, die zur Reduzierung des } \\
\text { Konfliktpotenzials beitragen, } \\
\text { z.B. Beruhigung sensibler Lebensräume }\end{array}$ & $\begin{array}{l}\text { Reduzierung der Störung sensibler } \\
\text { Lebensräume durch das individuelle Ver- } \\
\text { halten der Freizeitnutzer }\end{array}$ \\
\hline
\end{tabular}

Abbildung 27: Konflikttypen

Quelle: Eigene Zusammenstellung

Der zweite Konflikttyp setzt erst ein, wenn bereits Regelungen und Restriktionen existieren. Das Konfliktpotenzial leitet sich aus dem Gegensatz zwischen Nutzungsansprüchen und Nutzungsrestriktionen ab und verkörpert somit die Sicht der Nutzer. Gegenstand der Konflikte ist damit nicht die Ausübung der Freizeitaktivitäten an sich, sondern das Verhalten einzelner Freizeitnutzer aufgrund einer mangelnden Akzeptanz von Restriktionen oder auch einer unzureichende Information über die Regelungen (z.B. Verletzen des Wegegebotes, Nichteinhaltung zeitlicher Restriktionen). Wenngleich dies in der Regel nur einen kleinen Teil der Freizeitnutzer - Einzelpersonen und kleinere Gruppen - betrifft, kann dadurch die Störwirkung der Freizeitnutzung erheblich erhöht werden. Die Konfliktbewältigung richtet sich daher auf beide Aspekte in einer Art Doppelstrategie. Durch eine intensive Information und Aufklärung werden nicht nur die Regelungen selbst besser bekannt gemacht, sondern auch ökologische Zusammenhänge und die Auswirkungen bestimmter Verhaltensweisen auf die Wildtiere erläutert. Darüber hinaus sind gegenüber denjenigen, die Nutzungsrestriktionen bewusst übertreten, Sanktionen notwendig (vgl. Abbildung 27).

Beide Konflikttypen hängen in der Praxis häufig eng zusammen und stehen in einer Wechselwirkung zueinander. Das führt dazu, dass beispielsweise Ruhezonen für Wildtiere oder Wegegebote für bestimmte Freizeitaktivitäten nicht ihre volle Wirkung entfalten. Hinzu kommt, dass insbesondere der zweite Konflikttyp mit einer Beeinträchtigung anderer Freizeitaktivitäten verbunden sein kann, was zu Konflikten zwischen den verschiedenen Freizeitnutzergruppen führt. 
Ein weiterer wichtiger Aspekt ist die Beeinflussung der Bejagbarkeit des Wildes. Im Rahmen der genannten Konflikttypen kann es sein, dass die Tiere ihr Raum-Zeit-Verhalten soweit ändern, dass die Bejagung deutlich erschwert wird. Ebenso kann die Ausübung der Jagd durch das bloße Vorhandensein von Erholungssuchenden stark erschwert werden, da eine gefahrlose Schussabgabe nicht mehr gewährleistet ist. Beide Aspekte können bei erhöhtem Regulierungsbedarf einer Art massive Managementprobleme nach sich ziehen, obwohl die Art selbst durch die Freizeitaktivitäten nicht direkt negativ beeinflusst wird. 